\title{
13. DIAGENETIC ALTERATIONS AND GEOCHEMICAL TRENDS IN EARLY CRETACEOUS SHALLOW-WATER LIMESTONES OF ALLISON AND RESOLUTION GUYOTS (SITES 865 TO 868) ${ }^{1}$
}

\author{
Ursula Röhl ${ }^{2}$ and André Strasser ${ }^{3}$
}

\begin{abstract}
Cretaceous shallow-water limestones were recovered from two flat-topped submarine seamounts (guyots) in the area of the Mid-Pacific Mountains during Leg 143. In the center of Allison Guyot (Site 865), a nearly 700-m-thick sequence of upper Albian lagoonal limestones overlain by a 150-m-thick pelagic sedimentary cover of Paleogene to Quaternary age was drilled before terminating in basalt. A transect from the lagoon across the perimeter mound of Resolution Guyot was established by Sites 866 , 867 , and 868 . Hole $866 \mathrm{~A}$ resulted in a thick (more than $1600 \mathrm{~m}$ of carbonates overlying basalt) sequence of Hauterivian to Albian lagoonal to reefal limestones. Resolution Guyot bears only a thin pelagic cap of Maastrichtian to Pliocene age.

The diagenesis of the shallow-water Lower Cretaceous carbonates of Allison and Resolution guyots took place in response to changes in rock pore-fluid composition over time. Analyses of diagenetic variability in combination with geochemical studies allow for the reconstruction of chemical compositions and diagenetic pathways. The disappearance of the volcanic island is evidenced by the decreasing clay contents of the limestones. Major, minor, and trace element analysis of bulk samples illustrates this process in high resolution. We identified an excellent correspondence of cyclic distributions of phosphate, manganese, copper, and zinc with sequence-stratigraphic interpretations for Site 866: primary element compositions are probably related to sea-level variations. Early diagenetic alterations are documented by different cement and porosity types. Analyses of cement chemistry and stable isotope composition permit the reconstruction of marine-phreatic, meteoric-phreatic, and meteoric-vadose diagenetic environments, prior to the development of karst at the top of Hole 866 A and the strong late diagenetic dolomitization in its lower part.
\end{abstract}

\section{INTRODUCTION}

Ocean Drilling Program (ODP) Site 865 is located on Allison Guyot and Sites 866, 867, and 868 are on Resolution (formerly "Huevo") Guyot in the area of the Mid-Pacific Mountains (Fig. 1). Our main objectives were (1) the characterization of the chemical composition of the bulk sediment, (2) identification of diagenetic alterations, (3) lithological and chemical analyses of selected portions of the sediments (e.g., different cement types, allochems) to obtain details about the diagenetic pathways, (4) comparison of chemical data with microfacies and diagenetic features, and (5) classification of microfacies and diagenetic types according to their chemical compounds.

As the recovery of core was only about $15 \%$ on average, one must keep in mind that all resulting interpretations are limited. However, the total of $2500 \mathrm{~m}$ of shallow-water carbonates of Hauterivian to late Albian age, in combination with the excellent logging results, provided for reconstruction of the diagenetic history and chemical alteration.

\section{METHODS}

Our study began with the evaluation of macroscopic observations of the cores made during the cruise. Interpretation of the logging data played an important role in interpreting these sequences having mainly poor recovery (cf. Arnaud et al., this volume; Cooper et al., this volume).

Several hundred thin sections of samples from Leg 143 participants (Arnaud, Röhl, Strasser) were analyzed for the interpretation of microfacies and diagenetic history. The microfacies and facies zone assignments (Arnaud et al., this volume; Strasser et al., this volume)

\footnotetext{
' Winterer, L.A., Sager, W.W., Firth, J.V., and Sinton, J.M. (Eds.), 1995. Proc. ODP, Sci. Results, 143: College Station. TX (Ocean Drilling Program),

${ }^{2}$ Bundesanstalt für Geowissenschaften und Rohstoffe. Postfach 5101 53, D-30631 Hannover, Federal Republic of Germany.

${ }^{3}$ Institut de Géologie, Université de Fribourg. Pérolles, CH-1700 Fribourg, Switzerland.
}

were necessary for the shore-based diagenetic studies because primary facies variations are a major factor in determining the diagenetic pathways. For detailed studies, staining methods (Alizarin-Red-S), scanning electron microscope (SEM), and cathodoluminescence analysis (CL) were performed. X-ray-fluorescence analysis (XRF: $\mathrm{SiO}_{2}, \mathrm{TiO}_{2}, \mathrm{Al}_{2} \mathrm{O}_{3}, \mathrm{Fe}_{2} \mathrm{O}_{3}, \mathrm{MnO}, \mathrm{MgO}, \mathrm{CaO}, \mathrm{P}_{2} \mathrm{O}_{5}, \mathrm{SO}_{3}$. As, $\mathrm{Ba}, \mathrm{Ce}$, $\mathrm{Co}, \mathrm{Cr}, \mathrm{Cu}, \mathrm{La}, \mathrm{Ni}, \mathrm{Sr}, \mathrm{V}, \mathrm{Zn}, \mathrm{Zr}$, loss on ignition [LOI]) gave an overview of primary chemical composition and the subsequent diagenetic alterations. The analyses were performed on powder ignited at $1000^{\circ} \mathrm{C}$ and fused with a sample/flux ratio of 1:5 and calibrated against international standards. Accuracy was better than $2 \%$ for major elements and better than $5 \%$ for minor and trace elements. Major, minor, and trace elements $(\mathrm{Ca}, \mathrm{Mg}, \mathrm{Sr}, \mathrm{Fe}, \mathrm{Mn})$ in components and cements were quantitatively measured with an ARL-SEMQ electron-beam II microprobe. Parameter settings were as follows: beam diameter $30 \mu \mathrm{m}$, accelerator voltage $15 \mathrm{kV}$, sample current $20 \mathrm{nA}$ (dolomite standard), counting times $20 \mathrm{~s}$ (peak) and $10 \mathrm{~s}$ (background). Dolomite $(\mathrm{Ca}, \mathrm{Mg})$, siderite $(\mathrm{Mn}, \mathrm{Fe})$, and strontianite $(\mathrm{Sr})$ were used as standards. $\mathrm{K}_{\alpha}$-lines were measured for all elements except $\mathrm{Sr}\left(\mathrm{L}_{\alpha 1}\right)$. Measured values were corrected with the program MAGIC IV. Standard deviations $(1 \sigma)$ rarely exceeded $\pm 1 \%$ of the measured value. Stable isotopes $\left(\delta^{18} \mathrm{O}, \delta^{13} \mathrm{C}\right)$ were analyzed with a Finnigan Delta-S mass spectrometer. The standard deviations are $\pm 0.3 \% \circ\left(\delta^{13} \mathrm{C}\right)$ and $\pm 0.15 \%\left(\delta^{18} \mathrm{O}\right)$, respectively. A new (for the samples of Leg 143) created method for analyzing small sample amounts (10 mg) was used to determine $\mathrm{Ca}, \mathrm{Mg}, \mathrm{Sr}, \mathrm{Mn}, \mathrm{Fe}$, and $\mathrm{Zn}$ contents of carefully selected samples of cements (Elsholz, unpubl. data). A digestion by hydrochloric acid (10\%) and a buffering solution of Lanthan-chloride-7-hydrate was used. The element contents were determined by atomic absorption analysis (AA) by a Philips PU $9400 \mathrm{X}$ and inductively coupled plasma spectrometry (ICP) by a sequential spectrometer Philips PU 7000. X-ray diffraction (XRD, Philips PW 3710) analysis verified mineralogical compositions, especially the dolomite content and the $\mathrm{Ca} / \mathrm{Mg}$ ratio in both calcite and dolomite, and to confirm the occurrence of apatite and barite. The scanning electron microscope prints were taken with the CamScam microscope of the BGR. 


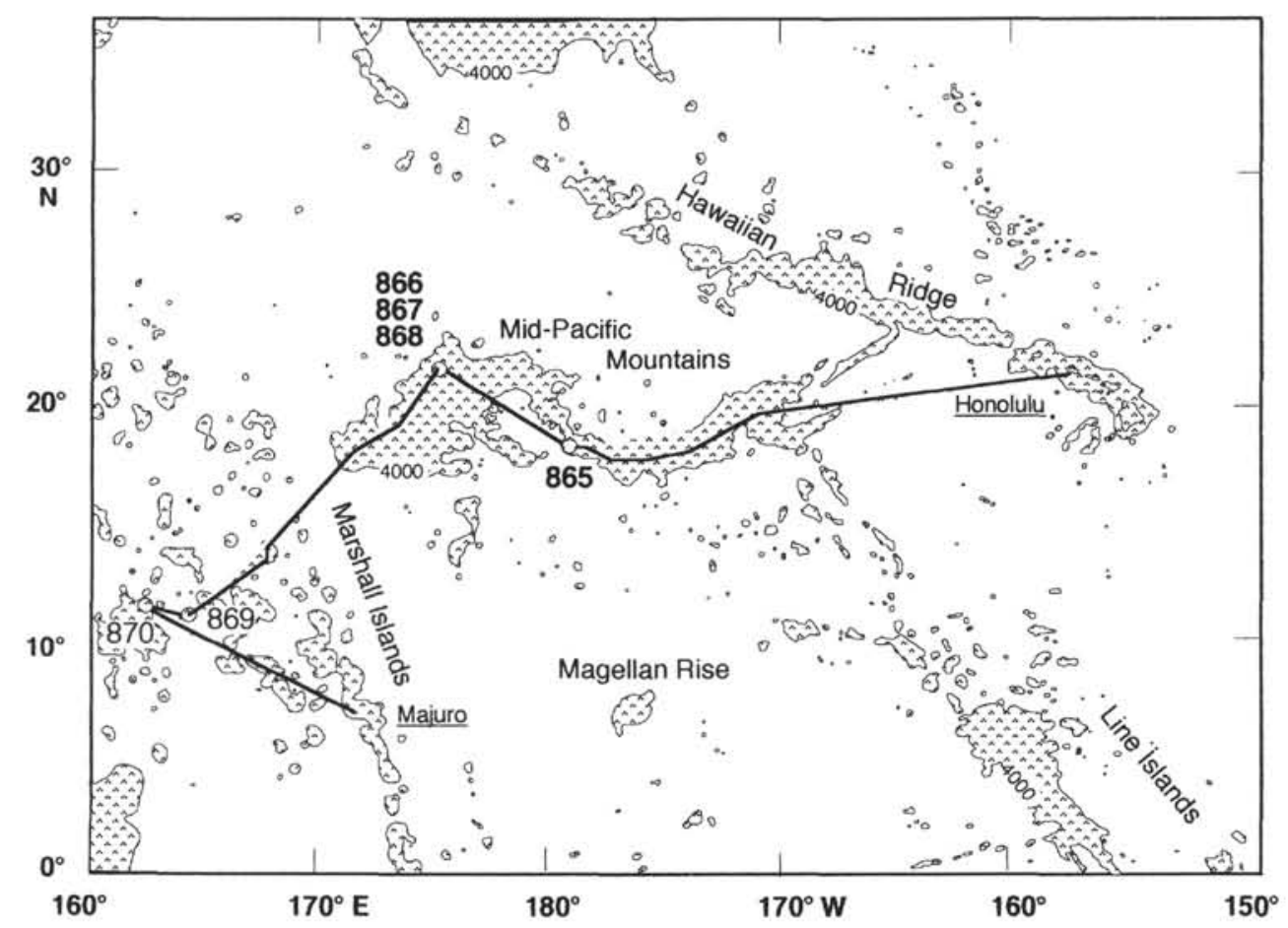

Figure 1. Location of Leg 143 drill sites and principal seamount chains (shallower than $4000 \mathrm{~m}$ ), in the western central Pacific Ocean. Line shows track of JOIDES Resolution, Sites 865 to 868 (bold) are discussed here.

\section{REGIONAL AND LITHOLOGICAL SETTINGS}

Northwestward motion of the Pacific Plate of about $30^{\circ}$ latitude since the Early Cretaceous has resulted in subduction of most Cretaceous sediments deposited in the Northern Hemisphere (Winterer, 1991). The Mid-Pacific Mountains are part of the chains and clusters of the Western Pacific seamounts (Sager, Winterer, Firth, et al., 1993). The seafloor near Resolution Guyot is probably of Jurassic age and originated by mid-plate volcanism near the ridge crest (Winterer et al., this volume). Because the volcanoes become younger eastward, the thickness of the carbonate caps decreases in this direction (Winterer et al., this volume). Allison Guyot $\left(179^{\circ} \mathrm{W}, 800\right.$-m-thick Albian limestones) and Resolution Guyot $\left(174^{\circ} \mathrm{E}, 1600\right.$-m-thick Hauterivian to late Albian shallow-water carbonates) fit into this general picture.

The lagoonal sediments of Allison Guyot are dominated by wackestones to packstones that are characterized by varying contents of foraminifers, mollusks, calcareous algae, and some clays (volcanic island stage) in the lower part, and wackestones to packstones with high-spired gastropods, sponges, and large sponge spicules in the upper part of the section. Resolution Guyot sediments from Hole 866A comprise different shallow-water limestones, oolites, and oncolites at the base, whereas peritidal carbonates punctuated by beach sediments and small coral and rudist bioherms dominate in the upper part. The sediments at Sites 867 and 868 indicate that the Resolution platform was rimmed by barrier islands and storm beaches and that rudist or sponge reefs were of only minor importance. Lithological variability, including detailed microfacies characterization and sequence stratigraphy of the Cretaceous shallow-water limestones, is described in detail by Arnaud et al. (this volume). Strasser et al. (this volume) have studied the meter-scale sequences of Sites 866, 867, and 868.

\section{DIAGENETIC SETTING}

Allison Guyot has been below sea level at least since the midTuronian (Site 865, Bralower and Mutterlose, this volume), and Resolution Guyot, at least since the Pliocene (Sager, Winterer, Firth, et al.,
1993). Because most of the sediments were deposited in very shallow water (Sager, Winterer, Firth, et al., 1993), episodic exposure in the meteoric diagenetic environment was highly probable. However, a period of subaerial exposure occurred during the end of late Albian to pre-mid-Turonian time, as evidenced by extensive subsurface karst (e.g., vugs, caves, and cavities filled by speleothems, van Waasbergen and Winterer, this volume). On the other hand, mineralization by phosphate and manganese occurs in the upper part of the shallowwater sequences, suggesting submarine hardgrounds (ODP Leg 143 Shipboard Scientific Party, 1993). Geophysical data from both guyots display karstified topography (van Waasbergen and Winterer, 1993).

None of the Cretaceous sediments has ever been buried deeper than $850 \mathrm{~m}$ at Allison guyot, or $1600 \mathrm{~m}$ at Resolution Guyot. The degree of lithification is largely a function of its original mineralogy (James and Bone, 1989): thus, most of the sediments drilled on Allison and Resolution guyots are well cemented, as they initially contained a high proportion of aragonitic components (e.g., dasycladacean algae, gastropods). The nonrecovered portions of the shallow-water sequences are probably less cemented. The oolitic grainstones at Hole 866A, Unit V, are poorly lithified, possibly owing to the original predominantly calcitic or Mg-calcitic composition (cf. Strasser and Jenkyns, this volume). The lower portion of Site 866, below $1050 \mathrm{~m}$ below sea-floor (mbsf), shows all transitions from scattered dolomite rhombs to destructive dolomitization. These dolosparstones (Wright, 1992) have been studied in detail by Flood and Chivas (this volume).

\section{RESULTS}

\section{Diagenetic Features and Interpretation}

The studied limestones were affected by generally strong diagenetic alteration. Especially in the uppermost parts of the drilled sections, late diagenetic alterations (van Waasbergen and Winterer, this volume) are responsible for substantial modification of the initial cement fabric and may have introduced unconformities in the diagenetic chronology. Independent of single facies type (Strasser et al., 
this volume; Arnaud et al., this volume) and lithification, the precipitation of cements in pores was only a minor factor during diagenetic alteration in most of the samples. Instead, different dissolutionderived porosities are of higher significance in the classification of diagenetic environments. Nevertheless, several different cement types could be identified that are discussed in relation to the marinephreatic, meteoric-phreatic, and meteoric-vadose diagenetic realms.

\section{Cements}

All cements are now of low-magnesium calcite mineralogy, and are mostly iron-poor, and nonluminescent, except those of the lower third section of Allison Guyot (Site 865, see chapter on AA/ICP chemical results). Marine-phreatic cements fringing components occur in most of the investigated thin sections. Comparable observations on dredge samples in the northwestern Pacific were made by Grötsch and Flügel (1992). Especially limestones having high primary intergranular porosity (grainstones) were obviously favorable to early diagenetic cementation and show excellent examples (see Pl. 1, Figs. 1 and 3, and Pl. 3, Fig. 2). Marine cements of primary aragonitic mineralogy, such as even-rim acicular and even-rim fibrous cement, are very rare (Pl. 3, Fig. 3) or absent. Aggregates were initially bound by organic filaments (Pl. 4, Fig. 2). An early compaction stage, which in some cases preceded any cementation, resulted in the overpacking and interpenetration of grains with little cement $(\mathrm{Pl}$. 4, Fig. 7) and the spalling of fringing cements (Pl. 1, Fig. 5) or shells (Pl. 5, Fig. 7). Syntaxial cementation surrounding echinoderm fragments prevented compaction in some pore spaces (Pl. 2, Figs. 1 and 2 ). A solution corona, a void that formed by selective micrite dissolution around an echinoderm ossicle (Walkden and Berry, 1984) and with precipitation of syntaxial cement into it, could not be identified. Partly bladed, even-rim cements are very common in all facies types (PI. 1, Figs. 3 and 4). Scalenohedral crystal terminations of even-rim cements are typical for grainstones and rudstones having relatively high interparticle porosity (Pl. 3). The distribution of scalenohedral crystals is variable and ranges from one or two tiny crystals on grain surfaces to complete envelopes of fossil fragments. The morphology of these cements resembles that of Pleistocene high-Mg calcite cements described by Vollbrecht and Meischner (1993).

Besides the original mineralogy, petrophysical properties equally influenced the diagenetic transformations (cf. Aissaoui, 1988). The rudist facies (Strasser et al., this volume) having relatively big fossil fragments, is dominated by dissolution. Only the micritized envelopes and early fringing cements commonly have been preserved (Pl. 3). Micritic meniscus cements are rare and found in grainstones of Site 866 (Pl. 2, Fig. 7). Peloidal cements occur in the interspaces of corals (Pl, 1, Fig. 6). They probably had an original high-Mg calcite mineralogy (Chafetz, 1986). Algal mats contain spherulites, with radial growth of calcite envelopes (Pl. 1, Fig. 7). Grainstones, with several hardground pieces, exhibit the strongest cementation (Pl. 3, Fig. 3). In general, blocky, pore-filling calcite cements are of minor importance. The blocky cement (Pl. 5, Fig. 5) in the lower portion of Site 866 is mainly of dolomitic mineralogy (see chapter on microprobe analyses). At least one initial phase of dolomitization, some of which grows in cracks $(\mathrm{Pl}$. 5, Fig. 4) and is related to minor scale sequences (cf. Strasser et al., this volume) and their clayey horizons (PI. 5, Fig. 1), took place very early. The partial dolomitization may be related to algal and microbial mats resulting from $\mathrm{Mg}^{2+}$ bound on organic complexes, which was released in pore water after decay of the organisms (cf. Koch et al., 1989). Some dolomite rhombs show signs of dissolution (PI. 5, Fig. 2 and 6).

Fringing (even-rim) cements and blocky calcite cements, partly in combination with burial overprinting (see chapter on stable isotopes), are more important in the lower portion of Site 865 (Unit IV, Fig. 2). In Hole 866A cementation (Fig. 3A), respectively, different cement types (Fig. 3B) are most prominent in the upper part of Unit VI, partly in the oolitic Unit V, and in Units VII and VIII (mainly dolomite cement, see above).
Because complete diagenetic sequences are absent and because of the strong overprinting by dissolution, our interpretation of diagenetic environments was limited. Even-rim fringing cements are most probably of marine-phreatic origin. Bladed, even-rim cements having scalenohedral crystal terminations occur as primary $\mathrm{Mg}$-calcite in the marinephreatic environment (e.g., Moore, 1989; Vollbrecht and Meischner, 1993, compare with Longman, 1980; Strasser and Davaud, 1986).

McKenzie et al. (1980) studied the scalenohedral cements in Paleogene shallow-water carbonates of the Emperor Seamounts, drilled during Deep Sea Drilling Project (DSDP) Leg 55. They found a high correlation between occurrence of scalenohedral cements and decreasing distance to the underlying basalt. Such a relationship could not be identified for the Allison and Resolution guyots limestones.

Desiccation cracks, bird's-eye structures, keystone vugs, meniscus, and peloidal cements indicate meteoric-vadose conditions. Glaebules reflect pedogenetic overprinting. Because the micritic cement may originally have been of aragonite, either high-Mg calcite, or low-Mg calcite mineralogy; either a marine or a meteoric-phreatic origin is possible.

The blocky, pore-filling calcite cements in different facies types suggest, according to their textures, a meteoric-phreatic to shallowburial origin (PI. 2, Fig. 4 and Pl. 3, Fig. 9). These cements may have been overprinted by seawater (see chapter on stable isotope studies and compare with Tucker [1990], Sun et al. [1992]). The blocky dolomite cements of the lower portion of Site 866 were precipitated from cold marine waters that percolated through the drowned carbonate platform below $2000 \mathrm{~m}$ (Flood and Chivas, this volume). A comparable origin was also proposed for the Eocene dolomites of Enewetak Atoll (Saller, 1984).

\section{Porosity}

Primary porosities in the recovered material are generally low and limited to interparticle porosities (according to Choquette and Pray, 1970) in weakly and noncemented oolitic grainstones (Pl. 2, Fig. 3, and Jenkyns and Strasser, this volume). Keystone vugs and bird's-eyes are found in oolites and algal laminites, respectively (cf. Arnaud et al., this volume, Strasser et al., this volume). Intraparticle porosity typically occurs in foraminifers, coral fragments, calcareous algae (Pl. 4, Fig. 1) and echinoderm fragments. Intercrystalline porosity was found in coarse dolomites (PI. 5, Fig. 3), some of which also show dissolved areas in the center of single crystals (intracrystalline porosity, Pl. 5, Fig. 6).

More widespread is small-scale dissolution, manifested as different types of secondary porosity types and therefore of importance for the definition of diagenetic pathways. Spatial variations are caused by the individual characteristics of allochems and cements, the fabric of the limestone, hydrology, and earlier diagenetic processes (Schroeder, 1988). All samples show some evidence of dissolution, which acted mainly fabric-selectively. Moldic porosity, which primarily originated from the selective removal of the former fossils by aragonite dissolution, is most common (Pl. 4, Figs. 4 and 5) in all facies types. This dissolution is local, and the degree of reduction of early secondary porosity by marine cementation is relatively low (Pl. 4, Fig. 9), which may be related to the mineralogy of the limestones. James and Bone (1989) discussed the presence of aragonite being the main driving force in meteoric diagenesis. Most of the aragonite in Leg 143 samples had probably already been transformed to calcite at the time of the origin of secondary porosity (cf. Budd, 1989).

According to Moore (1989), most shallow-water carbonate sequences bear the imprint of meteoric diagenesis. The guyot limestones also exhibit the influences of destructive solution by freshwater, which enlarged initial porosities (Pl. 4, Figs. 7 and 8 ) and resulted in the progressive destruction of fossils across all previous fabrics. Dissolution related to the lowering of $\mathrm{pH}$ following the decomposition of organic matter (cf. Miller, 1986) can be excluded, especially for the Site 866 samples, because the contents of organic matter in this site are, in general, relatively low (cf. Baudin et al., this volume). 


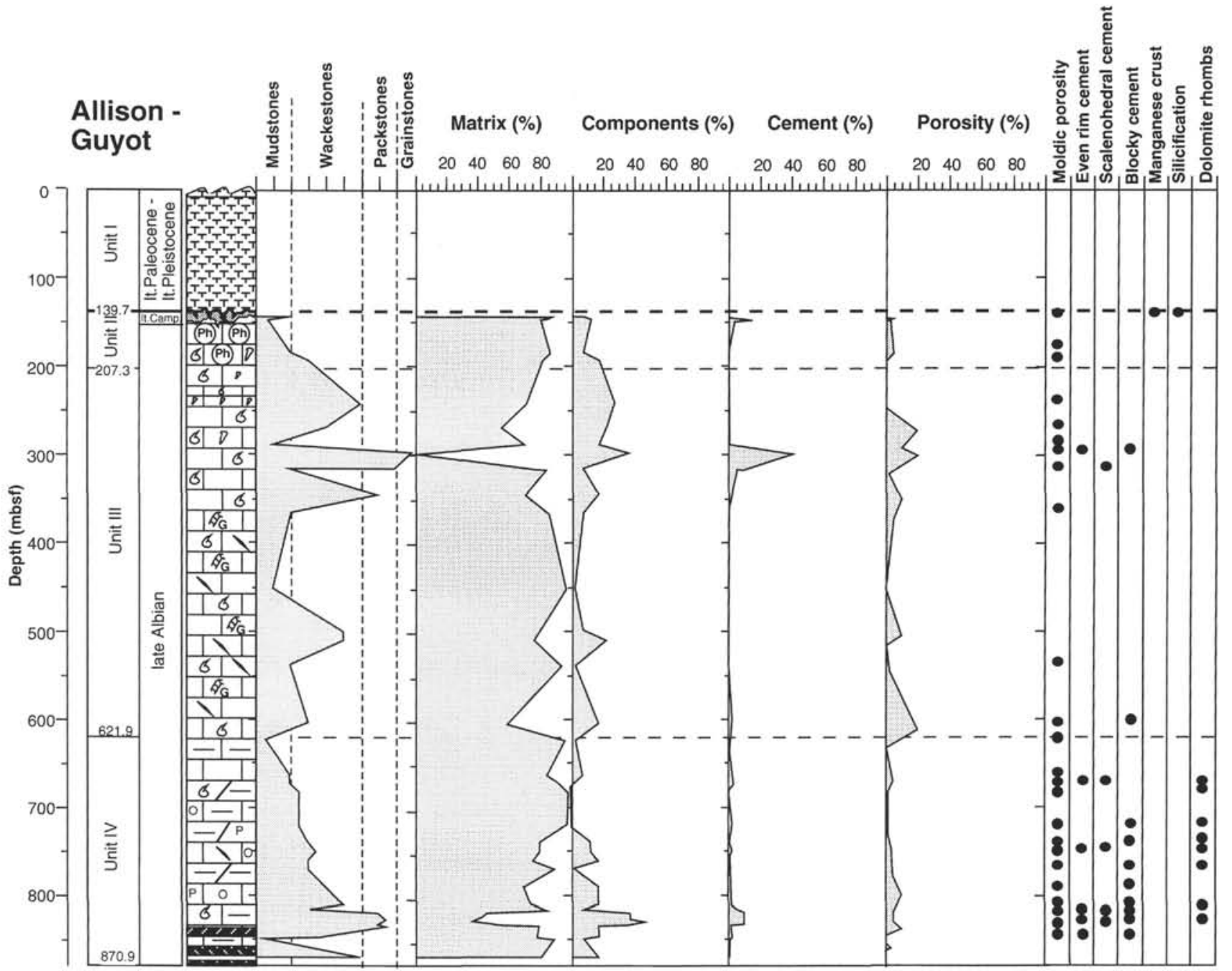

Figure 2. Stratigraphic distribution of matrix, components, cements, porosity, and diagenetic features in Hole 865A (Allison Guyot) as estimated from thin sections.

The stratigraphic distribution of porosity in Hole $865 \mathrm{~A}$ shows generally higher porosity values in Unit III in comparison with Unit IV (Fig. 2). The highest porosity values occur in Unit II. Moldic porosity was found throughout the section of Hole 866A (Fig. 3B), but is prominent in the oolitic Unit $\mathrm{V}$ and the grainstones to packstones of the upper portion of Unit VI. Samples from Site 867 (stormbeach sequences of Strasser et al., this volume) exhibit interparticle porosities up to $30 \mathrm{vol} \%$. The porosity is probably higher in the unrecovered part of the holes, especially in the upper karstified parts of Sites 865 to 868 . The circulation system of significant seawater flushing through the section proposed by Paull et al. (this volume) may be partly responsible for the development of higher porosity, whereas diagenetic alteration is directly related to the residence times and chemical evolution of waters. Paull et al. (this volume) estimated that the Allison carbonate platform is flushed by seawater no more rapidly than once every million years, but $\mathrm{Sr}$ isotope values of pore waters indicate that the host carbonates have not influence the pore water composition significantly.

\section{Geochemical Results and Interpretation}

\section{Bulk Chemistry}

\section{Site 865 (Allison Guyot)}

The abrupt change in the $\mathrm{Sr}$ and $\mathrm{Ba}$ contents between nannofossil/ foraminifer oozes of the pelagic cap (Unit I, Bralower et al., this vol- ume) and the Cretaceous shallow-water limestones is related to differences in lithology, age, and diagenetic alteration of the carbonates.

Unit II is characterized by penetrative phosphatization (up to $20.53 \% \mathrm{P}_{2} \mathrm{O}_{5}$ !, Fig. 4) and a manganese crust on top (Mn and associated elements: $\mathrm{Fe}, \mathrm{Cu}, \mathrm{Co}, \mathrm{Zn}$, for contents see Table 1). XRD analyses showed apatite, todorokite, and traces of quartz.

Unit III exhibits a relatively homogeneous carbonate distribution, with contents of more than $92 \% \mathrm{CaCO}_{3}$. The magnesium contents (mean $0.6 \% \mathrm{MgO}$ ) slightly decrease upward, which is related to the decreasing clay content that tracks the disappearance of the central volcanic island.

The element distribution in Unit IV is of wider variety as a result of the still-existing influence of the central volcanic island, resulting in more impure limestones and alternations of limestones with clays and/or marls (Figs. 4 and 5). $\mathrm{SiO}_{2}, \mathrm{Al}_{2} \mathrm{O}_{3}, \mathrm{TiO}_{2}, \mathrm{Fe}_{2} \mathrm{O}_{3}, \mathrm{MnO}, \mathrm{V}, \mathrm{Zn}$, $\mathrm{Cu}$, and $\mathrm{Co}$ values change cyclically with the lithologic variations in this unit. The $\mathrm{MgO}$ distribution reflects mainly clay. Although dolomite rhombs are very rare in thin sections, minor dolomite is also a possible source for magnesium.

\section{Site 866 (Resolution Guyot)}

The $\mathrm{CaCO}_{3}$ distribution (Fig. 6A) exhibits constantly high values in Units III and IV (between $90 \%$ and $98 \%$, Table 2), with only a slightly decreasing trend downward. The incision in the carbonate curve of Unit III results from a green clay layer $\left(4.4 \% \mathrm{CaCO}_{3}\right)$, and 


\begin{tabular}{|l|l|}
\hline & LEGEND \\
Rock and sediment types & Foraminiteral \\
nannofossil ooze
\end{tabular}

Figure 2 (continued).

the low carbonate contents in Units V and VI reflect algal laminite layers. The lower part of Unit VI and Units VII and VIII are characterized by the occurrence and increasing content of dolomite (up to $18.14 \% \mathrm{MgO}$, Fig. 6A, Flood and Chivas, this volume). XRD analyses of 35 dolomite-dominated bulk samples showed $\mathrm{Ca}: \mathrm{Mg}$ ratios between ${ }^{57} \mathrm{Ca} /{ }^{43} \mathrm{Mg}$ and ${ }^{54} \mathrm{Ca} /{ }^{46} \mathrm{Mg}$ : the $\mathrm{MgCO}_{3}$ content within the calcite lattice is between 3 and $5 \mathrm{wt} \%$.
Srand $\mathrm{Ba}$ are bound in the $\mathrm{CaCO}_{3}$ lattice and show an overall trend similar to that of $\mathrm{CaCO}_{3}$ (Fig. $6 \mathrm{~A}$ ), with maximum values in the algal laminite samples (up to $450 \mathrm{mg} / \mathrm{kg} \mathrm{Sr}$ and $22 \mathrm{mg} / \mathrm{kg} \mathrm{Ba}$ ). Relatively high $\mathrm{Sr}$ values may reflect an original aragonite mineralogy.

Phosphate $\left(\mathrm{P}_{2} \mathrm{O}_{5}\right.$, Fig. 6B) is mainly below the detection limit. As no samples were analyzed from the top part of the section, where we found penetrative phosphatization (Sager, Winterer, Firth, et al., 1993), only samples from four horizons (Samples 143-866A-35R-1, 33-35 cm; 143-866A-46R-2, 46-48 cm; 143-866A-71R-2, 71-72 $\mathrm{cm} ; 143-866 \mathrm{~A}-98 \mathrm{R}-1,108-110 \mathrm{~cm}$, Fig. 6B) show higher values, up to $0.27 \% \mathrm{P}_{2} \mathrm{O}_{5}$. Comparison of these phosphate "peaks" with results of biostratigraphy (Arnaud and Sliter, this volume) and interpretations of sequence stratigraphy (Arnaud et al., this volume) gives a good correlation of higher phosphate contents, with maximum flooding surfaces identified by the occurrence of nannofossils and pelagic foraminifers on one side and lithological sequences on the other side. The late diagenetic phosphogenesis of equatorial Pacific seamounts was studied by Hein et al. (1993).

$\mathrm{Al}_{2} \mathrm{O}_{3}$ and $\mathrm{SiO}_{2}\left(0.1 \%\right.$ to $14.28 \% \mathrm{Al}_{2} \mathrm{O}_{3}$ and $0.05 \%$ to $37.59 \%$ $\mathrm{SiO}_{2}$ ) are related to detrital minerals and reflect the amount of clay in the cores. Two overall "increasing clay" cycles are visible within Units VI and III (Fig. 6B). In general, the $\mathrm{Al}_{2} \mathrm{O}_{3}$ and $\mathrm{SiO}_{2}$ curves fit well. An exception is visible in Cores 143-866A-108R through $-111 \mathrm{R}$, where we found up to $68.95 \% \mathrm{SiO}_{2}$, but only background $\mathrm{Al}_{2} \mathrm{O}_{3}$ contents. These higher $\mathrm{SiO}_{2}$ values correlate with the presence of fragments of volcanic glass observed in thin section (Arnaud et al., this volume).

The distributions of manganese oxide (Fig. 6B), and also of $\mathrm{Cu}$ and $\mathrm{Zn}$ (Fig. 6C), exhibit a cyclic behavior in Units V through VII. They could be classified as "increasing upward" cycles on a 50 - to $100-\mathrm{m}$ scale (see arrows in Figs. 6B and 6C). Each of the cycles is supported by four to six samples, which show the trend clearly. It seems that these elements and, respectively, compounds, show a sedimentary trend (cyclically increasing clay) in higher resolution in the facies of Units V through VIII than do Al and $\mathrm{Si}$, which normally are clay markers. Cooper (this volume) and Arnaud et al. (this volume) found Milankovitch cycles on a meter scale, as well as larger cycles of up to tenths (?hundreds) of meters in thickness, which are integrated in a sequence stratigraphic framework. Comparison of chemical data (unfortunately, geochemical logging failed) with cycles identified by Arnaud et al. (this volume) shows that, in general, $\mathrm{MnO}$ peaks are linked to the most restricted facies (i.e., algal mat facies) and, therefore, correspond to sequence boundaries (e.g., Sb 5 , 7,10 , and 15 of Arnaud et al., this volume), whereas $\mathrm{MnO}$ minima correlate with maximum flooding surfaces (e.g., mfs 10, 11, and 15 of Arnaud et al., this volume). Thus, the flux of manganese seems related to terrestrial runoff, which is strongest when relative sea level is low. Some cycles show a concordance with increasing resistivity and density trends in the logs (Cooper et al., this volume).

$\mathrm{SO}_{3}(0.02 \%$ to $2.32 \%$ ) and $\mathrm{V}$ (1 to $162 \mathrm{mg} / \mathrm{kg}$ ) obviously are related to organic matter. A comparison of our results with the data of Baudin et al. (this volume) shows that $\mathrm{SO}_{3}$ and $\mathrm{V}$ peaks are correlated with intervals that contain relatively high amounts of total organic carbon (TOC). Therefore, algal laminites and black packstones are the facies types having increased $\mathrm{SO}_{3}$ and $\mathrm{V}$ contents (Fig. 6C).

\section{Site 867 (Resolution Guyot)}

The uppermost samples are from limestones overlain by a manganese crust ( $\mathrm{Mn}, \mathrm{Fe}, \mathrm{Ba}, \mathrm{Cu}$; for contents see Table 3 ). These carbonates have been partly silicified (up to $70 \% \mathrm{SiO}_{2}$, XRD studies identified well crystallized quartz) and contain some clusters of barite needles (Pl. 5, Fig. 8, see high Ba contents in Table 3). Subunit IIA is also characterized by phosphatized limestones. XRF studies obtained values up to $20 \% \mathrm{P}_{2} \mathrm{O}_{5}$, and $\mathrm{XRD}$ analysis verified the occurrence of apatite (Pl. 5, Fig. 9). Limestones having high $\mathrm{CaCO}_{3}$ contents of more than $95 \%$ occur throughout the section. 


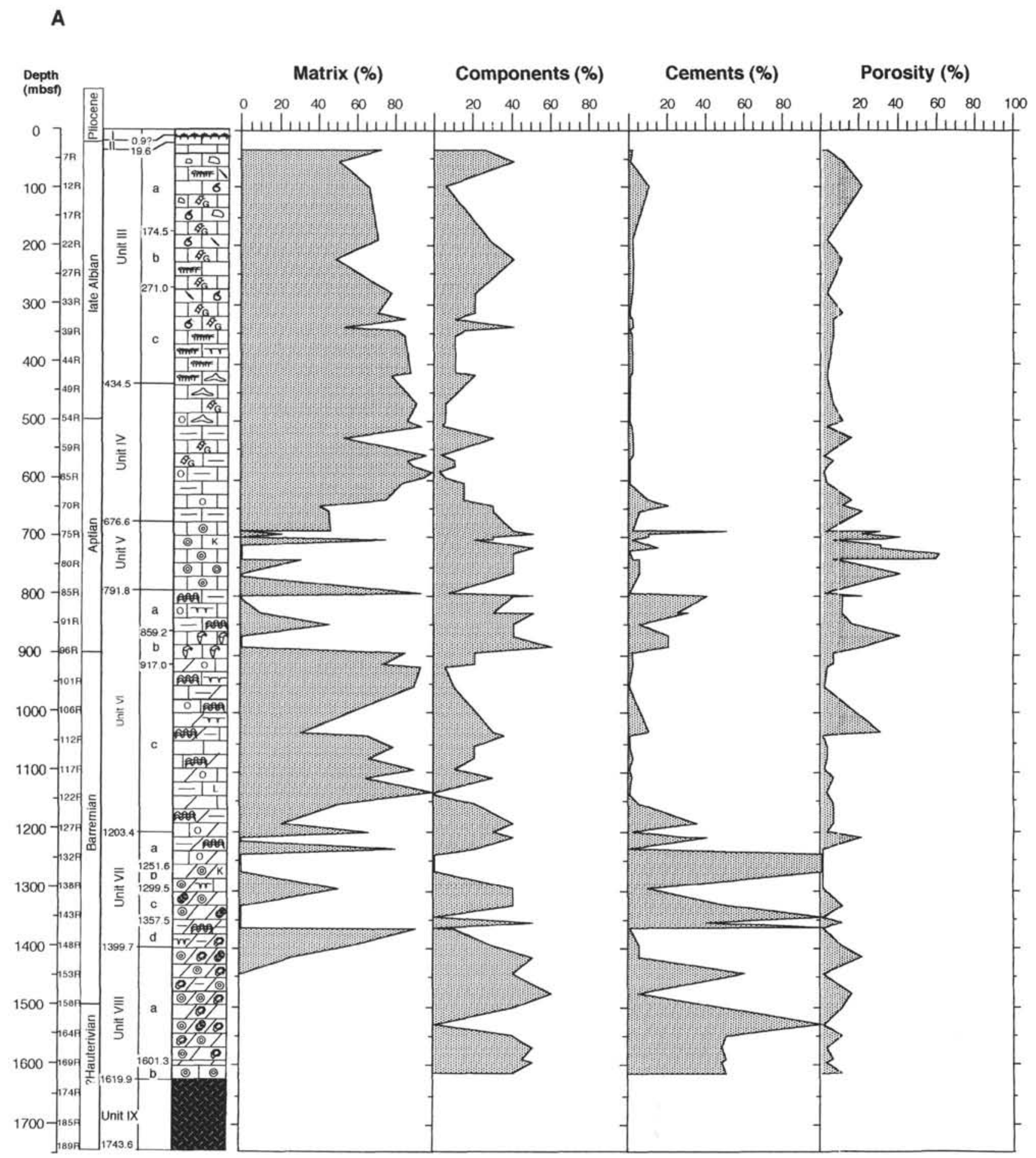

Figure 3. A. Stratigraphic distribution of matrix, components, cements, and porosity in Hole 866A (Resolution Guyot) as estimated from thin sections. B. Diagenetic features and limestone classification after Wright (1992). 


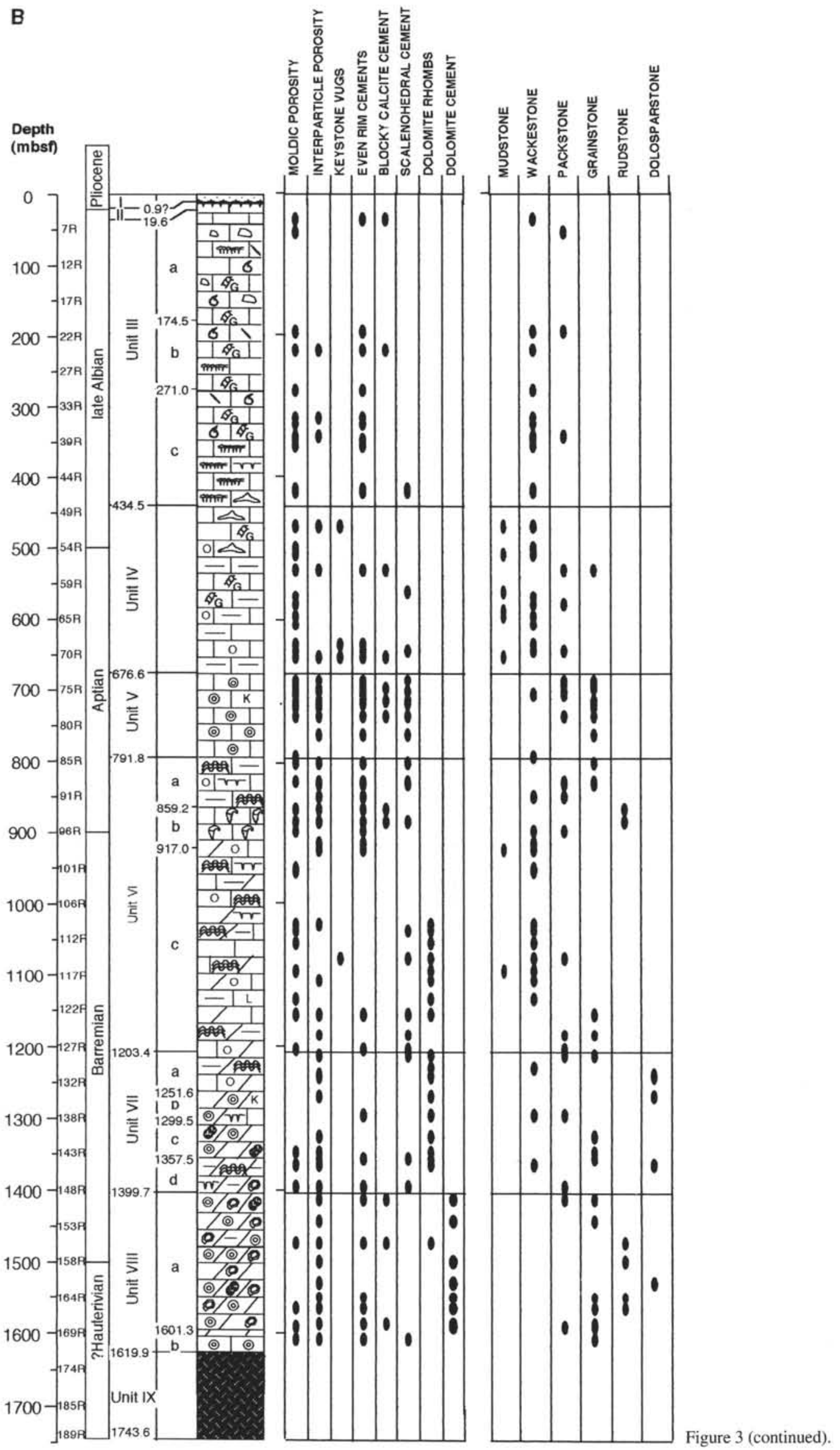


Table 1. Major, minor, and trace elements (XRF) of Hole 865A.

\begin{tabular}{|c|c|c|c|c|c|c|c|c|c|c|}
\hline $\begin{array}{l}\text { Core, section, } \\
\text { interval }(\mathrm{cm})\end{array}$ & $\begin{array}{l}\text { Depth } \\
\text { (mbsf) }\end{array}$ & $\begin{array}{l}\mathrm{SiO}_{2} \\
(\%)\end{array}$ & $\begin{array}{l}\mathrm{TiO}_{2} \\
(\%)\end{array}$ & $\begin{array}{l}\mathrm{Al}_{2} \mathrm{O}_{3} \\
(\%)\end{array}$ & $\begin{array}{l}\mathrm{Fe}_{2} \mathrm{O}_{3} \\
(\%)\end{array}$ & $\begin{array}{c}\mathrm{MnO} \\
(\%)\end{array}$ & $\begin{array}{l}\mathrm{MgO} \\
(\%)\end{array}$ & $\begin{array}{l}\mathrm{CaO} \\
(\%)\end{array}$ & $\begin{array}{l}\mathrm{P}_{2} \mathrm{O}_{5} \\
(\%)\end{array}$ & $\begin{array}{l}\mathrm{SO}_{3} \\
(\%)\end{array}$ \\
\hline \multicolumn{11}{|l|}{ 143-865A- } \\
\hline $1 \mathrm{R}-2,60-62$ & 2.1 & 1.25 & 0.026 & 0.43 & 0.29 & 0.039 & 0.36 & 51.64 & 0.03 & 0.23 \\
\hline $2 \mathrm{R}-1,82-84$ & 6.52 & 0.14 & 0.009 & 0.08 & 0.25 & 0.027 & 0.47 & 51.5 & 0.01 & 0.33 \\
\hline $2 \mathrm{R}-2,78-80$ & 7.98 & 0.13 & 0.009 & 0.07 & 0.21 & 0.024 & 0.49 & 51.11 & 0.01 & 0.32 \\
\hline $2 \mathrm{R}-3,31-35$ & 9.01 & 0.17 & 0.008 & 0.08 & 0.49 & 0.032 & 0.35 & 52.64 & 0.01 & 0.24 \\
\hline $2 \mathrm{R}-4,83-86$ & 11.03 & 0.11 & 0.006 & 0.08 & 0.05 & 0.025 & 0.31 & 54.73 & 0.01 & 1.44 \\
\hline $4 \mathrm{R}-1,58-60$ & 24.78 & 0.19 & 0.006 & 0.1 & 0.06 & 0.026 & 0.38 & 52.77 & 0.01 & 2.54 \\
\hline $4 \mathrm{R}-2,80-84$ & 26.5 & 0.19 & 0.006 & 0.09 & 0.05 & 0.021 & 0.33 & 53.28 & 0.02 & 0.3 \\
\hline $4 R-3,40-44$ & 27.6 & 0.27 & 0.007 & 0.11 & 0.06 & 0.025 & 0.42 & 51.72 & 0.02 & 0.34 \\
\hline $5 R-1,44-46$ & 34.14 & 0.24 & 0.006 & 0.1 & 0.06 & 0.023 & 0.46 & 50.78 & 0.02 & 0.4 \\
\hline $5 R-2,44-47$ & 35.14 & 0.3 & 0.007 & 0.12 & 0.06 & 0.026 & 0.43 & 51.25 & 0.02 & 0.35 \\
\hline $5 R-3,43-47$ & 35.71 & 0.23 & 0.006 & 0.1 & 0.05 & 0.02 & 0.62 & 50.09 & 0.02 & 1.39 \\
\hline $5 R-4,41-45$ & 37.26 & 0.32 & 0.008 & 0.13 & 0.07 & 0.025 & 0.36 & 53.1 & 0.04 & 1.63 \\
\hline $5 R-5,43-45$ & 38.28 & 0.3 & 0.007 & 0.12 & 0.06 & 0.028 & 0.39 & 53.18 & 0.03 & 0.36 \\
\hline $5 R-6,42-45$ & 38.85 & 0.32 & 0.008 & 0.13 & 0.07 & 0.027 & 0.33 & 53.36 & 0.03 & 0.29 \\
\hline $6 \mathrm{R}-1,56-60$ & 43.76 & 0.27 & 0.007 & 0.1 & 0.06 & 0.022 & 0.48 & 51.81 & 0.02 & 0.36 \\
\hline $6 \mathrm{R}-2,39-43$ & 45.09 & 0.31 & 0.007 & 0.12 & 0.06 & 0.025 & 0.43 & 52.63 & 0.02 & 0.33 \\
\hline $7 R-2,40-42$ & 54.1 & 0.16 & 0.006 & 0.08 & 0.04 & 0.019 & 0.46 & 52.44 & 0.02 & 1.09 \\
\hline $7 \mathrm{R}-3,36-38$ & 54.63 & 0.19 & 0.006 & 0.09 & 0.05 & 0.021 & 0.53 & 50.64 & 0.02 & 1.4 \\
\hline $7 R-4,40-42$ & 56.19 & 0.16 & 0.005 & 0.08 & 0.05 & 0.02 & 0.54 & 50.24 & 0.02 & 0.58 \\
\hline $7 R-5,41-43$ & 57.3 & 0.28 & 0.007 & 0.12 & 0.06 & 0.02 & 0.37 & 53.32 & 0.03 & 0.32 \\
\hline $8 \mathrm{R}-1,94-99$ & 63.24 & 0.2 & 0.006 & 0.1 & 0.05 & 0.025 & 0.31 & 53.79 & 0.03 & 0.24 \\
\hline $8 \mathrm{R}-2,22-27$ & 64.02 & 0.28 & 0.007 & 0.12 & 0.06 & 0.02 & 0.46 & 52.29 & 0.03 & 0.34 \\
\hline $9 \mathrm{R}-1,60-62$ & 72.4 & 0.12 & 0.005 & 0.07 & 0.04 & 0.015 & 0.8 & 47.8 & 0.05 & 1.16 \\
\hline $9 \mathrm{R}-2,80-82$ & 74.1 & 0.1 & 0.005 & 0.06 & 0.05 & 0.017 & 0.6 & 50.38 & 0.06 & 1.04 \\
\hline $9 \mathrm{R}-3,20-22$ & 75 & 0.14 & 0.005 & 0.07 & 0.05 & 0.019 & 0.58 & 51.49 & 0.06 & 0.55 \\
\hline $13 \mathrm{R}-1,47-51$ & 111.3 & 0.1 & 0.005 & 0.06 & 0.04 & 0.01 & 0.46 & 51.87 & 0.05 & 0.41 \\
\hline $13 R-2,48-52$ & 112.8 & 0.12 & 0.007 & 0.07 & 0.05 & 0.009 & 0.31 & 54.25 & 0.06 & 0.23 \\
\hline $13 R-3,47-52$ & 114.3 & 0.14 & 0.006 & 0.07 & 0.05 & 0.01 & 0.38 & 54.03 & 0.07 & 0.26 \\
\hline $13 \mathrm{R}-4,48-52$ & 115.8 & 0.1 & 0.005 & 0.07 & 0.04 & 0.011 & 0.38 & 53.34 & 0.06 & 0.62 \\
\hline $13 R-5,48-52$ & 117.3 & 0.11 & 0.005 & 0.06 & 0.04 & 0.012 & 0.53 & 51.03 & 0.06 & 0.73 \\
\hline $13 \mathrm{R}-6,49-53$ & 118.8 & 0.14 & 0.006 & 0.07 & 0.05 & 0.013 & 0.36 & 53.27 & 0.06 & 0.43 \\
\hline $13 \mathrm{R}-7,49-52$ & 120.3 & 0.14 & 0.005 & 0.08 & 0.12 & 0.014 & 0.3 & 54.09 & 0.08 & 0.32 \\
\hline $14 \mathrm{R}-1,4-6$ & 120.5 & 0.01 & 0.004 & 0.06 & 0.32 & 0.019 & 0.47 & 51.89 & 0.04 & 0.37 \\
\hline $14 \mathrm{R}-2,58-62$ & 122.6 & 0.11 & 0.005 & 0.07 & 0.06 & 0.012 & 0.46 & 52.24 & 0.04 & 0.35 \\
\hline $14 \mathrm{R}-3,67-70$ & 124.2 & 0.12 & 0.005 & 0.09 & 0.05 & 0.01 & 0.36 & 52.8 & 0.09 & 0.57 \\
\hline $14 \mathrm{R}-4,58-61$ & 125.6 & 0.1 & 0.005 & 0.06 & 0.04 & 0.009 & 0.4 & 52.36 & 0.07 & 0.47 \\
\hline $15 \mathrm{R}-1,78-82$ & 130.9 & 0.15 & 0.006 & 0.08 & 0.06 & 0.009 & 0.31 & 53.74 & 0.1 & 0.31 \\
\hline $15 \mathrm{R}-2,43-46$ & 132 & 0.1 & 0.005 & 0.06 & 0.05 & 0.013 & 0.4 & 51.5 & 0.14 & 0.36 \\
\hline $15 \mathrm{R}-3,29-32$ & 133.4 & 0.1 & 0.005 & 0.07 & 0.05 & 0.012 & 0.41 & 53.33 & 0.11 & 0.27 \\
\hline $15 R-4,79-82$ & 135.4 & 0.16 & 0.006 & 0.08 & 0.05 & 0.011 & 0.51 & 51.98 & 0.1 & 0.29 \\
\hline $15 R-5,42-45$ & 136.5 & 0.21 & 0.007 & 0.09 & 0.06 & 0.01 & 0.35 & 53.81 & 0.14 & 0.21 \\
\hline $15 \mathrm{R}-6,58-61$ & 138.2 & 0.16 & 0.006 & 0.07 & 0.06 & 0.013 & 0.45 & 52.52 & 0.1 & 0.32 \\
\hline $15 R-7,16-20$ & 139.3 & 0.28 & 0.009 & 0.11 & 0.09 & 0.013 & 0.39 & 52.72 & 0.24 & 0.27 \\
\hline $16 \mathrm{R}-1,114-118$ & 136.2 & 0.16 & 0.008 & 0.08 & 0.28 & 0.015 & 0.38 & 53.59 & 0.14 & 0.2 \\
\hline $16 \mathrm{R}-2,79-83$ & 137.4 & 0.16 & 0.007 & 0.08 & 0.07 & 0.014 & 0.23 & 54.67 & 0.2 & 0.09 \\
\hline $16 \mathrm{R}-3,64-68$ & 138.7 & 0.16 & 0.006 & 0.07 & 0.11 & 0.015 & 0.45 & 52.57 & 0.18 & 0.27 \\
\hline $16 \mathrm{R}-4,40-44$ & 140 & 0.16 & 0.007 & 0.08 & 0.12 & 0.013 & 0.33 & 54.15 & 0.19 & 0.15 \\
\hline $16 \mathrm{R}-5,40-43$ & 141.5 & 0.16 & 0.009 & 0.07 & 0.09 & 0.024 & 0.52 & 51.98 & 0.36 & 0.33 \\
\hline $17 \mathrm{R}-\mathrm{CC}, 5-8$ & 139.75 & 4.03 & 0.441 & 1.06 & 6.65 & 11.6 & 1.25 & 33.55 & 20.53 & 1.33 \\
\hline 19R-CC, 8-9 & 149.48 & 1.19 & 0.016 & 0.21 & 0.37 & 0.096 & 0.55 & 53.85 & 0.18 & 0.02 \\
\hline $23 \mathrm{R}-\mathrm{CC}, 6-7$ & 188.06 & 0.69 & 0.013 & 0.25 & 0.2 & 0.02 & 0.59 & 54.06 & 0.01 & 0.02 \\
\hline $28 \mathrm{R}-1,26-28$ & 236.56 & 0.26 & 0.007 & 0.09 & 0.07 & 0.007 & 0.51 & 54.83 & 0 & 0.02 \\
\hline $32 \mathrm{R}-\mathrm{CC}, 2-3$ & 274.9 & 0.48 & 0.013 & 0.2 & 0.1 & 0.009 & 0.62 & 53.8 & 0 & 0.02 \\
\hline $34 \mathrm{R}-1,8-9$ & 294.3 & 0.19 & 0.005 & 0.07 & 0.05 & 0.009 & 0.66 & 54.74 & 0 & 0.02 \\
\hline $39 \mathrm{R}-\mathrm{CC}, 28-29$ & 342.2 & 0.29 & 0.009 & 0.13 & 0.08 & 0.005 & 0.6 & 54.55 & 0 & 0.02 \\
\hline $40 \mathrm{R}-\mathrm{CC}, 24-26$ & 351.8 & 0.14 & 0.006 & 0.07 & 0.06 & 0.005 & 0.59 & 54.51 & 0 & 0.02 \\
\hline $42 \mathrm{R}-\mathrm{CC}, 7-9$ & 370.9 & 0.16 & 0.009 & 0.09 & 0.05 & 0.006 & 0.61 & 54.3 & 0 & 0.02 \\
\hline $44 \mathrm{R}-\mathrm{CC}, 9-11$ & 390.2 & 0.3 & 0.013 & 0.13 & 0.09 & 0.006 & 0.62 & 54.13 & 0 & 0.02 \\
\hline $52 \mathrm{R}-\mathrm{CC}, 6-8$ & 467.4 & 0.22 & 0.007 & 0.1 & 0.06 & 0.006 & 0.66 & 54.12 & 0 & 0.02 \\
\hline 56R-CC, 7-10 & 506 & 0.39 & 0.014 & 0.17 & 0.1 & 0.005 & 0.71 & 53.93 & 0 & 0.05 \\
\hline $58 \mathrm{R}-\mathrm{CC}, 12-14$ & 525.3 & 0.13 & 0.007 & 0.08 & 0.05 & 0.005 & 0.66 & 54.44 & 0 & 0.02 \\
\hline $60 \mathrm{R}-\mathrm{CC}, 11-13$ & 544.6 & 0.23 & 0.011 & 0.11 & 0.06 & 0.005 & 0.67 & 54.3 & 0.03 & 0.02 \\
\hline 66R-CC, $35-37$ & 602.9 & 0.18 & 0.01 & 0.06 & 0.06 & 0.005 & 0.73 & 53.97 & 0 & 0.07 \\
\hline $73 \mathrm{R}-1,26-28$ & 670.4 & 27.71 & 1.666 & 10.23 & 4.63 & 0.022 & 2.82 & 20.36 & 0.1 & 4.79 \\
\hline $76 \mathrm{R}-1,24-26$ & 699.3 & 0.58 & 0.018 & 0.25 & 0.13 & 0.005 & 0.91 & 53.27 & 0 & 0.34 \\
\hline $78 \mathrm{R}-1,59-60$ & 718.9 & 4.82 & 0.221 & 1.75 & 1.06 & 0.018 & 3.85 & 45.34 & 0 & 1.63 \\
\hline $79 \mathrm{R}-1,48-49$ & 728.6 & 0.76 & 0.032 & 0.32 & 0.17 & 0.008 & 0.86 & 53.13 & 0 & 0.27 \\
\hline $80 \mathrm{R}-1,32-35$ & 738 & 2.22 & 0.144 & 0.99 & 0.53 & 0.015 & 0.93 & 51.89 & 0 & 0.68 \\
\hline $81 \mathrm{R}-1,22-24$ & 747.6 & 0.28 & 0.011 & 0.14 & 0.08 & 0.007 & 0.9 & 53.85 & 0 & 0.17 \\
\hline $82 \mathrm{R}-1,48-49$ & 757.6 & 1.84 & 0.098 & 0.81 & 0.46 & 0.031 & 5.97 & 46.23 & 0.02 & 0.72 \\
\hline $83 R-C C, 17-20$ & 767 & 1.29 & 0.112 & 0.68 & 0.3 & 0.01 & 0.74 & 52.7 & 0 & 0.45 \\
\hline $85 R-2,65-68$ & 788.1 & 3.75 & 0.332 & $\begin{array}{l}0.30 \\
1.3\end{array}$ & 1.12 & 0.017 & 0.75 & 49.87 & 0 & 1.94 \\
\hline $86 \mathrm{R}-3,68-69$ & 799.4 & 2.18 & 0.433 & 1.4 & 0.87 & 0.02 & 0.8 & 50.52 & 0 & 1.19 \\
\hline $88 R-1,50-52$ & 815.6 & 1.86 & 0.223 & 1.32 & 1.0 & 0.016 & 0.78 & 51.58 & 0 & 1.66 \\
\hline $89 \mathrm{R}-2,58-60$ & 826.8 & 6.26 & 1.664 & 4.65 & 7.42 & 0.064 & 1.8 & 39.45 & 0.04 & 0.71 \\
\hline $90 \mathrm{R}-2,47-49$ & 833.4 & 4.85 & 0.638 & 2.63 & 27 & 0.05 & 0.97 & 46.22 & 0.03 & 3.99 \\
\hline $90 \mathrm{R}-3,42-44$ & 834.7 & 1.95 & 0.204 & 1.03 & 2.78 & 0.087 & 0.99 & 49.54 & 0.05 & 5.02 \\
\hline $91 \mathrm{R}-3,130-132$ & 845.2 & 6.86 & 0.999 & 2.94 & 2.3 & 0.098 & 1.9 & 44.66 & 0.06 & 0.94 \\
\hline $91 R-4,67-70$ & 846 & 2.96 & 0.279 & 1.32 & 0.36 & 0.05 & 0.77 & 51.45 & 0 & 0.02 \\
\hline $92 \mathrm{R}-2,26-28$ & 848.9 & 6.28 & 1.399 & 4.11 & 3.55 & 0.09 & 1.01 & 41.52 & 0.02 & 4.8 \\
\hline $92 \mathrm{R}-3,37-40$ & 849.9 & 9.57 & 1.485 & 4.38 & 2.06 & 0.054 & 1.34 & 42.43 & 0.07 & 1.06 \\
\hline $94 \mathrm{R}-3,129-131$ & 867.2 & 11.52 & 0.668 & 3.83 & 3.98 & 0.112 & 1.57 & 39.47 & 0.04 & 3.79 \\
\hline
\end{tabular}


DIAGENETIC ALTERATIONS AND GEOCHEMICAL TRENDS

Table 1 (continued).

\begin{tabular}{|c|c|c|c|c|c|c|c|c|c|c|c|}
\hline$\underset{(\mathrm{mg} / \mathrm{kg})}{\mathrm{As}}$ & $\begin{array}{c}\mathrm{Ba} \\
(\mathrm{mg} / \mathrm{kg})\end{array}$ & $\underset{(\mathrm{mg} / \mathrm{kg})}{\mathrm{Ce}}$ & $\begin{array}{c}\text { Co } \\
(\mathrm{mg} / \mathrm{kg})\end{array}$ & $\begin{array}{c}\mathrm{Cr} \\
(\mathrm{mg} / \mathrm{kg})\end{array}$ & $\underset{(\mathrm{mg} / \mathrm{kg})}{\mathrm{Cu}}$ & $\underset{(\mathrm{mg} / \mathrm{kg})}{\mathrm{La}}$ & $\begin{array}{c}\mathrm{Ni} \\
(\mathrm{mg} / \mathrm{kg})\end{array}$ & $\begin{array}{c}\mathrm{Sr} \\
(\mathrm{mg} / \mathrm{kg})\end{array}$ & $\underset{(\mathrm{mg} / \mathrm{kg})}{\mathrm{V}}$ & $\underset{(\mathrm{mg} / \mathrm{kg})}{\mathrm{Zn}}$ & $\begin{array}{c}\mathrm{Zr} \\
(\mathrm{mg} / \mathrm{kg})\end{array}$ \\
\hline 1 & 295 & 20 & 8 & 1 & 16 & 16 & 9 & 1077 & 14 & 31 & 8 \\
\hline 1 & 94 & 21 & 9 & i & 15 & 24 & 1 & 850 & 1 & 198 & 1 \\
\hline 1 & 78 & 34 & 1 & 1 & 18 & 18 & i & 860 & $i$ & 144 & 1 \\
\hline i & 242 & 19 & 1 & 36 & 21 & 14 & 1 & 833 & i & 1263 & $i$ \\
\hline 5 & 350 & 1 & 1 & 1 & 16 & 35 & 1 & 811 & 1 & 1 & i \\
\hline 1 & 653 & 1 & $i$ & $i$ & 11 & 26 & $i$ & 821 & $i$ & 12 & $i$ \\
\hline$i$ & 654 & 31 & 1 & 1 & 10 & 1 & 1 & 853 & 1 & 9 & 1 \\
\hline 1 & 903 & 16 & i & 1 & 17 & 12 & 1 & 796 & 1 & 11 & $i$ \\
\hline 1 & 891 & 24 & 1 & 1 & 13 & 18 & $i$ & 829 & 1 & 12 & 1 \\
\hline 1 & 954 & 18 & 1 & 1 & 19 & 11 & 1 & 827 & 1 & 9 & 1 \\
\hline 1 & 782 & 22 & 1 & 1 & 20 & 10 & $i$ & 780 & 1 & 13 & i \\
\hline$i$ & 1061 & 1 & 1 & 1 & 10 & 27 & 1 & 797 & $i$ & 48 & $i$ \\
\hline 1 & 1241 & 1 & i & 1 & 13 & 5 & $i$ & 862 & 1 & 32 & $i$ \\
\hline 1 & 1193 & 21 & 1 & 1 & 10 & 9 & 8 & 830 & 1 & 23 & 1 \\
\hline$i$ & 1020 & 1 & 8 & 1 & 10 & 15 & 1 & 776 & 1 & 19 & 1 \\
\hline$i$ & 1323 & 20 & 1 & 1 & 29 & 17 & $i$ & 734 & 10 & 16 & 1 \\
\hline$i$ & 954 & 1 & 1 & 1 & 18 & 28 & 1 & 696 & 1 & 15 & 1 \\
\hline 1 & 999 & i & i & 1 & 29 & 1 & 1 & 753 & 1 & 12 & $i$ \\
\hline 8 & 992 & 1 & 1 & 1 & 21 & 1 & 1 & 690 & i & 22 & 1 \\
\hline 1 & 1092 & 25 & 1 & 1 & 30 & 7 & 1 & 777 & $i$ & 31 & 1 \\
\hline 1 & 1270 & 1 & 10 & 1 & 15 & 5 & i & 777 & 1 & 12 & 1 \\
\hline 1 & 1073 & 26 & 9 & 1 & 11 & 32 & 1 & 730 & 1 & 13 & $i$ \\
\hline 1 & 972 & 26 & 1 & 1 & 53 & 1 & 1 & 597 & 1 & 11 & 1 \\
\hline 1 & 717 & 21 & 1 & 1 & 45 & 20 & 40 & 522 & 1 & 10 & 1 \\
\hline 1 & 878 & 20 & i & i & 31 & 20 & 1 & 469 & 1 & 9 & 1 \\
\hline 1 & 795 & 1 & 1 & 1 & 44 & 20 & 1 & 797 & 1 & 11 & 1 \\
\hline 1 & 943 & 1 & 1 & 1 & 29 & 5 & 1 & 863 & 1 & 13 & 1 \\
\hline 1 & 974 & 1 & 1 & 1 & 39 & 7 & 1 & 877 & 1 & 15 & $i$ \\
\hline 1 & 841 & 18 & i & 1 & 23 & 27 & 1 & 817 & 1 & 10 & 1 \\
\hline 1 & 895 & 23 & $i$ & i & 32 & 1 & 1 & 803 & 1 & 28 & $i$ \\
\hline 1 & 1063 & 31 & 1 & 1 & 29 & 14 & 1 & 812 & 1 & 30 & 1 \\
\hline 1 & 1009 & 17 & 1 & 1 & 11 & 22 & 1 & 830 & 1 & 11 & 1 \\
\hline 1 & 599 & 1 & 1 & 38 & 38 & 22 & 1 & 789 & 13 & 230 & $i$ \\
\hline 1 & 477 & 29 & 1 & 12 & 25 & 11 & 10 & 659 & 1 & 29 & 1 \\
\hline i & 753 & 24 & i & 1 & 17 & 17 & 1 & 776 & $i$ & 12 & $i$ \\
\hline i & 568 & 1 & 1 & 1 & 16 & 28 & 1 & 715 & 1 & 33 & $\mathrm{i}$ \\
\hline 5 & 699 & i & i & $\mathrm{i}$ & 20 & 1 & $i$ & 682 & 1 & 11 & 1 \\
\hline 1 & 834 & 1 & 7 & $i$ & 18 & 12 & 1 & 653 & 12 & 8 & $\mathrm{i}$ \\
\hline 1 & 606 & 21 & 1 & $i$ & 12 & 24 & $i$ & 645 & 1 & 11 & 1 \\
\hline 1 & 827 & 25 & 1 & 1 & 18 & 19 & 1 & 624 & 1 & 40 & $i$ \\
\hline 1 & 1185 & 1 & 1 & 1 & 18 & 17 & 1 & 647 & 1 & 11 & 1 \\
\hline 1 & 982 & 28 & i & i & 17 & 17 & 1 & 609 & $i$ & 8 & 1 \\
\hline 1 & 1274 & 31 & 1 & 1 & 10 & 35 & 1 & 664 & 1 & 9 & 1 \\
\hline 5 & 920 & 20 & 1 & 23 & 15 & 33 & 1 & 652 & 1 & 30 & 1 \\
\hline 1 & 899 & 41 & 1 & 1 & 40 & 32 & $i$ & 681 & 1 & 20 & 1 \\
\hline 1 & 878 & 1 & 1 & 1 & 24 & 24 & 1 & 638 & 1 & 20 & 1 \\
\hline 6 & 844 & 16 & 7 & 1 & 25 & 1 & 1 & 632 & 1 & 17 & 1 \\
\hline 1 & 532 & 15 & 1 & 1 & 15 & 21 & 10 & 687 & 11 & 9 & 9 \\
\hline 129 & 5698 & 544 & 1896 & 90 & 972 & 529 & 3645 & 172 & 5400 & 565 & 377 \\
\hline 8 & 91 & 1 & 22 & 37 & 37 & 17 & 33 & 172 & 29 & 18 & 1 \\
\hline 1 & 19 & 1 & 9 & 1 & 18 & 23 & 7 & 166 & 11 & 8 & 1 \\
\hline 1 & 16 & 1 & 1 & 1 & 12 & 23 & 1 & 307 & 1 & 1 & 1 \\
\hline$i$ & 16 & $i$ & 1 & 1 & 1 & 18 & 1 & 170 & 18 & 9 & 1 \\
\hline 1 & 14 & 1 & $i$ & $i$ & i & 12 & 1 & 171 & 1 & 11 & 1 \\
\hline 1 & 23 & 30 & i & 1 & 16 & 1 & 1 & 176 & 12 & 8 & 1 \\
\hline 1 & 14 & 1 & 1 & $i$ & 1 & 26 & $i$ & 165 & 1 & 1 & 1 \\
\hline 1 & 12 & 1 & 1 & 1 & 1 & 30 & 1 & 179 & 1 & 1 & $i$ \\
\hline 1 & 16 & 16 & 1 & 8 & 10 & 18 & 1 & 171 & i & 1 & 1 \\
\hline 1 & 13 & 1 & i & 7 & 16 & 12 & 1 & 162 & 14 & 7 & 1 \\
\hline 1 & 13 & 24 & 1 & $i$ & 1 & 1 & 1 & 214 & 16 & 1 & 1 \\
\hline 1 & 13 & 29 & 1 & 12 & 12 & 14 & 1 & 195 & 1 & 7 & i \\
\hline 5 & 13 & 1 & 1 & 1 & 13 & 1 & 1 & 175 & 29 & 12 & 1 \\
\hline 1 & 16 & $i$ & $i$ & $i$ & 1 & 15 & $i$ & 171 & 1 & 14 & 1 \\
\hline 49 & 117 & 29 & 12 & 88 & 45 & 54 & 50 & 182 & 115 & 51 & 190 \\
\hline 6 & 13 & 1 & 1 & 1 & 1 & 12 & 26 & 256 & 32 & 8 & 1 \\
\hline 11 & 38 & $i$ & 1 & 14 & 31 & 23 & 11 & 198 & 40 & 18 & 48 \\
\hline 1 & 17 & $i$ & 1 & 1 & 27 & 11 & 8 & 212 & 12 & 13 & 13 \\
\hline 1 & 26 & i & 1 & 12 & 17 & 13 & 1 & 204 & 20 & 10 & 25 \\
\hline 1 & 16 & i & i & 15 & 16 & 29 & 1 & 243 & 1 & 10 & 1 \\
\hline 1 & 38 & $i$ & 1 & 7 & 37 & 20 & 13 & 201 & 14 & 23 & 18 \\
\hline 1 & 26 & 23 & 1 & 1 & 20 & 17 & 1 & 199 & 1 & 18 & 15 \\
\hline 1 & 42 & 28 & i & 25 & 33 & 15 & i & 245 & 25 & 18 & 31 \\
\hline 8 & 37 & 28 & 7 & 35 & 37 & 17 & 1 & 225 & 23 & 26 & 34 \\
\hline 1 & 31 & 18 & 1 & 22 & 32 & 40 & 7 & 295 & 25 & 8 & 30 \\
\hline 7 & 52 & 46 & 19 & 131 & 38 & 33 & 27 & 247 & 127 & 41 & 94 \\
\hline 8 & 36 & 1 & 8 & 47 & 1 & 28 & 16 & 345 & 29 & 12 & 51 \\
\hline 6 & 31 & 17 & 1 & 14 & 30 & 8 & 1 & 412 & 19 & 16 & 16 \\
\hline 1 & 40 & 1 & 1 & 75 & 27 & 23 & 23 & 361 & 62 & 25 & 66 \\
\hline i & 23 & i & $i$ & 25 & 17 & 28 & 1 & 232 & 48 & 1 & 26 \\
\hline 20 & 47 & 29 & $i$ & 95 & 16 & 21 & 29 & 371 & 81 & 20 & 80 \\
\hline 10 & 30 & 1 & 27 & 100 & 22 & 31 & 60 & 184 & 134 & 12 & 80 \\
\hline 8 & 56 & 1 & 1 & 47 & 15 & 25 & 10 & 430 & 41 & 24 & 53 \\
\hline
\end{tabular}


Table 2. Major, minor, and trace elements (XRF) in Hole 866A.

\begin{tabular}{|c|c|c|c|c|c|c|c|c|c|c|}
\hline $\begin{array}{l}\text { Core, section, } \\
\text { interval }(\mathrm{cm})\end{array}$ & $\begin{array}{l}\text { Depth } \\
\text { (mbsf) }\end{array}$ & $\begin{array}{l}\mathrm{SiO}_{2} \\
(\%)\end{array}$ & $\begin{array}{l}\mathrm{TiO}_{2} \\
(\%)\end{array}$ & $\begin{array}{c}\mathrm{Al}_{2} \mathrm{O}_{3} \\
(\%)\end{array}$ & $\begin{array}{c}\mathrm{Fe}_{2} \mathrm{O}_{3} \\
(\%)\end{array}$ & $\begin{array}{c}\mathrm{MnO} \\
(\%)\end{array}$ & $\begin{array}{l}\mathrm{MgO} \\
(\%)\end{array}$ & $\begin{array}{l}\mathrm{CaO} \\
(\%)\end{array}$ & $\begin{array}{l}\mathrm{P}_{2} \mathrm{O}_{5} \\
(\%)\end{array}$ & $\begin{array}{l}\mathrm{SO}_{3} \\
(\%)\end{array}$ \\
\hline \multicolumn{11}{|l|}{ 143-866A- } \\
\hline $6 \mathrm{R}-\mathrm{CC}, 2-3$ & 38.42 & 0.78 & 0.013 & 0.27 & 0.17 & 0.007 & 0.64 & 54.08 & 0 & 0.02 \\
\hline 8R-CC, 3-5 & 57.33 & 0.46 & 0.006 & 0.15 & 0.06 & 0.004 & 0.54 & 54.28 & 0 & 0.02 \\
\hline $10 \mathrm{R}-\mathrm{CC}, 4-5$ & 76.94 & 0.68 & 0.007 & 0.17 & 0.06 & 0.005 & 0.51 & 54.16 & 0 & 0.02 \\
\hline $19 \mathrm{R}-\mathrm{CC}, 3-4$ & 164.83 & 0.34 & 0.010 & 0.16 & 0.06 & 0.004 & 0.50 & 54.51 & 0 & 0.02 \\
\hline $25 R-1.53-55$ & 223.23 & 0.22 & 0.005 & 0.08 & 0.04 & 0.004 & 0.58 & 54.32 & 0 & 0.02 \\
\hline $31 \mathrm{R}-1,56-58$ & 281.16 & 0.18 & 0.008 & 0.11 & 0.06 & 0.005 & 0.54 & 54.67 & 0 & 0.02 \\
\hline $35 \mathrm{R}-1,33-35$ & 319.23 & 0.11 & 0.005 & 0.08 & 0.04 & 0.004 & 0.50 & 54.55 & 0.02 & 0.02 \\
\hline $35 \mathrm{R}-1,33-35$ & 319.23 & 0.39 & 0.012 & 0.20 & 0.06 & 0.004 & 0.44 & 54.53 & 0.27 & 0.02 \\
\hline $36 \mathrm{R}-1,21-23$ & 328.71 & 0.21 & 0.007 & 0.12 & 0.05 & 0.004 & 0.57 & 54.43 & 0.01 & 0.02 \\
\hline $40 R-C C, 36-38$ & 357.66 & 0.26 & 0.010 & 0.16 & 0.05 & 0.005 & 0.52 & 54.43 & 0 & 0.02 \\
\hline $39 \mathrm{R}-1,35-36$ & 348.05 & 0.15 & 0.007 & 0.10 & 0.05 & 0.005 & 0.49 & 54.93 & 0 & 0.02 \\
\hline $46 \mathrm{R}-2,46-48$ & 417.26 & 0.42 & 0.027 & 0.34 & 0.07 & 0.007 & 0.45 & 54.69 & 0.07 & 0.02 \\
\hline $48 \mathrm{R}-1,30-32$ & 434.70 & 0.19 & 0.009 & 0.13 & 0.20 & 0.004 & 0.54 & 54.29 & 0 & 0.07 \\
\hline 52R-CC, $18-19$ & 473.18 & 0.38 & 0.014 & 0.20 & 0.10 & 0.005 & 0.71 & 54.03 & 0 & 0.15 \\
\hline $55 \mathrm{R}-\mathrm{CC}, 30-31$ & 502.20 & 0.18 & 0.008 & 0.12 & 0.09 & 0.008 & 0.52 & 54.48 & 0 & 0.08 \\
\hline $58 \mathrm{R}-1,72-75$ & 531.72 & 0.12 & 0.006 & 0.08 & 0.05 & 0.005 & 0.56 & 54.15 & 0 & 0.14 \\
\hline $61 \mathrm{R}-1,103-10$ & 560.93 & 0.24 & 0.009 & 0.13 & 0.07 & 0.005 & 0.66 & 53.73 & 0 & 0.27 \\
\hline $62 R-2,21-23$ & 571.31 & 0.41 & 0.018 & 0.19 & 0.12 & 0.006 & 0.73 & 53.34 & 0 & 0.40 \\
\hline $64 \mathrm{R}-1,69-71$ & 589.69 & 0.28 & 0.010 & 0.15 & 0.08 & 0.004 & 0.71 & 53.46 & 0 & 0.36 \\
\hline $65 \mathrm{R}-1,57-60$ & 599.17 & 0.16 & 0.008 & 0.10 & 0.06 & 0.004 & 0.69 & 53.79 & 0 & 0.26 \\
\hline $70 \mathrm{R}-1,1-3$ & 646.91 & 2.84 & 0.149 & 0.97 & 0.79 & 0.006 & 0.80 & 50.59 & 0 & 0.74 \\
\hline $71 \mathrm{R}-1,64-67$ & 657.14 & 0.38 & 0.012 & 0.16 & 0.13 & 0.005 & 0.67 & 52.47 & 0 & 0.87 \\
\hline $71 \mathrm{R}-2,71-72$ & 658.49 & 37.59 & 0.971 & 14.28 & 8.12 & 0.028 & 3.45 & 2.49 & 0.02 & 2.32 \\
\hline $74 \mathrm{R}-2,94-97$ & 687.72 & 0.05 & 0.005 & 0.06 & 0.04 & 0.005 & 0.64 & 54.63 & 0 & 0.15 \\
\hline $75 R-3,67-69$ & 698.21 & 0.05 & 0.006 & 0.06 & 0.05 & 0.004 & 0.72 & 54.69 & 0 & 0.15 \\
\hline $76 \mathrm{R}-2,26-29$ & 706.46 & 2.10 & 0.069 & 1.06 & 0.29 & 0.006 & 0.95 & 51.79 & 0 & 0.43 \\
\hline $77 \mathrm{R}-1,59-60$ & 715.09 & 0.05 & 0.005 & 0.02 & 0.05 & 0.005 & 0.59 & 54.66 & 0 & 0.11 \\
\hline $78 R-2,85-87$ & 726.34 & 0.05 & 0.005 & 0.06 & 0.04 & 0.004 & 0.69 & 54.78 & 0 & 0.11 \\
\hline $79 R-2,104-10$ & 736.34 & 0.05 & 0.004 & 0.06 & 0.04 & 0.004 & 0.63 & 54.75 & 0 & 0.09 \\
\hline $80 \mathrm{R}-1,32-34$ & 743.82 & 0.05 & 0.005 & 0.05 & 0.04 & 0.005 & 0.64 & 54.56 & 0 & 0.06 \\
\hline $82 \mathrm{R}-2,45-48$ & 764.63 & 0.05 & 0.005 & 0.06 & 0.04 & 0.004 & 0.60 & 54.71 & 0 & 0.02 \\
\hline $85 R-2,140-14$ & 794.65 & 0.05 & 0.005 & 0.06 & 0.04 & 0.005 & 0.74 & 55.15 & 0 & 0.10 \\
\hline $86 \mathrm{R}-2,72-74$ & 803.72 & 0.22 & 0.008 & 0.12 & 0.07 & 0.005 & 0.72 & 54.01 & 0 & 0.22 \\
\hline $89 \mathrm{R}-1,39-40$ & 830.99 & 0.59 & 0.013 & 0.25 & 0.13 & 0.008 & 0.84 & 53.40 & 0 & 0.15 \\
\hline $91 \mathrm{R}-1,50-51$ & 850.00 & 0.80 & 0.023 & 0.34 & 0.18 & 0.005 & 0.95 & 51.67 & 0 & 0.91 \\
\hline $95 \mathrm{R}-1,21-24$ & 888.21 & 0.05 & 0.004 & 0.05 & 0.03 & 0.004 & 0.71 & 54.93 & 0 & 0.02 \\
\hline $97 \mathrm{R}-1,16-18$ & 907.56 & 0.21 & 0.009 & 0.11 & 0.09 & 0.005 & 0.99 & 53.90 & 0 & 0.18 \\
\hline $98 \mathrm{R}-1,108-110$ & 918.08 & 5.70 & 0.226 & 2.08 & 1.18 & 0.008 & 1.17 & 46.98 & 0.08 & 1.71 \\
\hline $100 \mathrm{R}-1,31-33$ & 933.71 & 1.89 & 0.045 & 0.71 & 0.31 & 0.007 & 1.05 & 52.32 & 0 & 0.3 \\
\hline $105 \mathrm{R}-1,82-84$ & 982.12 & 0.14 & 0.008 & 0.09 & 0.05 & 0.004 & 0.83 & 54.57 & 0 & 0.05 \\
\hline $103 \mathrm{R}-1,60-62$ & 963.00 & 0.22 & 0.009 & 0.13 & 0.07 & 0.005 & 0.79 & 53.93 & 0 & 0.3 \\
\hline $108 \mathrm{R}-1,55-57$ & 1010.75 & 0.05 & 0.006 & 0.07 & 0.05 & 0.006 & 0.8 & 54.12 & 0 & 0.12 \\
\hline $111 \mathrm{R}-1,49-50$ & 1039.79 & 0.57 & 0.015 & 0.24 & 0.11 & 0.005 & 1.01 & 53.56 & 0 & 0.66 \\
\hline $115 \mathrm{R}-1,22-26$ & 1078.12 & 68.95 & 0.007 & 0.05 & 0.05 & 0.004 & 0.27 & 15.87 & 0 & 0.61 \\
\hline $117 \mathrm{R}-1,44-47$ & 1097.54 & 0.32 & 0.011 & 0.12 & 0.07 & 0.005 & 1.11 & 53.65 & 0 & 1.07 \\
\hline $118 \mathrm{R}-1,144-146$ & 1108.24 & 2.59 & 0.058 & 0.93 & 0.47 & 0.009 & 1.22 & 49.51 & 0 & 1.64 \\
\hline $121 \mathrm{R}-1,56-58$ & 1136.26 & 1.42 & 0.022 & 0.50 & 0.36 & 0.006 & 13.19 & 38.46 & 0 & 0.28 \\
\hline $123 \mathrm{R}-1,55-57$ & 1155.65 & 0.16 & 0.006 & 0.07 & 0.05 & 0.004 & 8.61 & 45.14 & 0 & 0.21 \\
\hline $126 \mathrm{R}-2,32-33$ & 1185.61 & 0.05 & 0.005 & 0.06 & 0.05 & 0.004 & 0.9 & 54.25 & 0 & 0.18 \\
\hline $129 \mathrm{R}-1,77-78$ & 1213.67 & 2.03 & 0.127 & 0.76 & 0.67 & 0.007 & 10.8 & 40.20 & 0 & 1.35 \\
\hline $131 \mathrm{R}-1,12-14$ & 1232.32 & 1.60 & 0.067 & 0.55 & 0.34 & 0.006 & 15.23 & 35.93 & 0 & 1.08 \\
\hline $132 \mathrm{R}-1,40-41$ & 1242.30 & 2.08 & 0.069 & 0.78 & 0.41 & 0.005 & 17.41 & 32.98 & 0 & 0.48 \\
\hline $133 \mathrm{R}-2,108-1$ & 1254.18 & 0.05 & 0.006 & 0.02 & 0.04 & 0.003 & 18.14 & 34.08 & 0 & 0.25 \\
\hline $137 \mathrm{R}-2,43-45$ & 1291.55 & 0.05 & 0.006 & 0.02 & 0.04 & 0.004 & 17.27 & 35.18 & 0 & 0.19 \\
\hline $139 \mathrm{R}-1,45-47$ & 1309.65 & 0.05 & 0.005 & 0.06 & 0.04 & 0.005 & 1.08 & 53.99 & 0 & 0.15 \\
\hline I4IR-CC, $18-19$ & 1328.78 & 0.05 & 0.006 & 0.02 & 0.04 & 0.003 & 16.77 & 35.87 & 0 & 0.09 \\
\hline $143 \mathrm{R}-1,70-72$ & 1348.60 & 0.05 & 0.004 & 0.02 & 0.04 & 0.005 & 1.70 & 53.18 & 0 & 0.08 \\
\hline $145 \mathrm{R}-1,82-84$ & 1367.62 & 3.64 & 0.013 & 0.08 & 0.08 & 0.006 & 17.51 & 32.91 & 0 & 0.61 \\
\hline $147 \mathrm{R}-1,1-3$ & 1386.11 & 0.35 & 0.015 & 0.16 & 0.12 & 0.005 & 1.42 & 52.75 & 0 & 0.6 \\
\hline $147 \mathrm{R}-1,21-22$ & 1386.31 & 0.65 & 0.036 & 0.26 & 0.29 & 0.007 & 1.49 & 52.68 & 0 & 0.77 \\
\hline $147 \mathrm{R}-1,41-43$ & 1386.51 & 0.41 & 0.025 & 0.16 & 0.14 & 0.006 & 1.04 & 53.10 & 0 & 0.45 \\
\hline $147 \mathrm{R}-1,49-52$ & 1386.59 & 0.17 & 0.008 & 0.09 & 0.07 & 0.006 & 1.63 & 53.41 & 0 & 0.32 \\
\hline $147 \mathrm{R}-1,59-64$ & 1386.69 & 0.29 & 0.019 & 0.13 & 0.10 & 0.006 & 1.77 & 52.36 & 0 & 0.33 \\
\hline $147 \mathrm{R}-1,75-78$ & 1386.85 & 0.31 & 0.020 & 0.15 & 0.11 & 0.006 & 1.54 & 52.85 & 0 & 0.3 \\
\hline $147 \mathrm{R}-1,88-91$ & 1386.98 & 2.55 & 0.087 & 0.98 & 0.53 & 0.008 & 2.54 & 49.38 & 0 & 1.04 \\
\hline $147 \mathrm{R}-1,95-98$ & 1387.05 & 0.28 & 0.014 & 0.13 & 0.09 & 0.007 & 0.88 & 54.23 & 0 & 0.35 \\
\hline $148 R-4,35-37$ & 1399.65 & 3.04 & 0.143 & 1.21 & 0.59 & 0.015 & 0.83 & 50.87 & 0 & 0.84 \\
\hline $150 \mathrm{R}-1,9-11$ & 1415.19 & 0.05 & 0.006 & 0.06 & 0.05 & 0.006 & 5.11 & 49.01 & 0 & 0.23 \\
\hline $152 \mathrm{R}-2,93-95$ & 1436.86 & 0.16 & 0.012 & 0.09 & 0.07 & 0.005 & 0.92 & 53.61 & 0 & 0.21 \\
\hline $155 \mathrm{R}-3,9-11$ & 1466.42 & 0.05 & 0.007 & 0.05 & 0.04 & 0.005 & 0.95 & 53.85 & 0 & 0.17 \\
\hline $157 \mathrm{R}-2,52-54$ & 1484.78 & 0.15 & 0.016 & 0.08 & 0.06 & 0.005 & 0.98 & 54.23 & 0 & 0.19 \\
\hline $159 \mathrm{R}-2,65-67$ & 1503.05 & 0.05 & 0.008 & 0.07 & 0.05 & 0.006 & 1.72 & 53.16 & 0 & 0.22 \\
\hline $159 \mathrm{R}-1,87-89$ & 1501.77 & 0.05 & 0.009 & 0.02 & 0.05 & 0.004 & 13.79 & 39.47 & 0 & 0.17 \\
\hline $162 R-2,74-78$ & 1533.19 & 0.17 & 0.012 & 0.08 & 0.09 & 0.005 & 18.10 & 34.26 & 0 & 0.23 \\
\hline $164 \mathrm{R}-2,140-42$ & 1552.64 & 1.16 & 0.041 & 0.23 & 0.21 & 0.005 & 8.21 & 44.42 & 0 & 0.46 \\
\hline $166 \mathrm{R}-1,50-54$ & 1569.80 & 0.18 & 0.011 & 0.09 & 0.06 & 0.006 & 5.83 & 48.25 & 0 & 0.18 \\
\hline $168 \mathrm{R}-1,108-10$ & 1591.18 & 0.11 & 0.014 & 0.07 & 0.07 & 0.005 & 13.70 & 39.47 & 0 & 0.15 \\
\hline
\end{tabular}


DIAGENETIC ALTERATIONS AND GEOCHEMICAL TRENDS

Table 2 (continued).

\begin{tabular}{|c|c|c|c|c|c|c|c|c|c|}
\hline $\begin{array}{l}\text { LOI } \\
(\%)\end{array}$ & $\begin{array}{c}\mathrm{Ba} \\
(\mathrm{mg} / \mathrm{kg})\end{array}$ & $\underset{(\mathrm{mg} / \mathrm{kg})}{\mathrm{Ce}}$ & $\begin{array}{c}\mathrm{Cr} \\
(\mathrm{mg} / \mathrm{kg})\end{array}$ & $\underset{(\mathrm{mg} / \mathrm{kg})}{\mathrm{Cu}}$ & $\underset{(\mathrm{mg} / \mathrm{kg})}{\mathrm{La}}$ & $\begin{array}{c}\mathrm{Sr} \\
(\mathrm{mg} / \mathrm{kg})\end{array}$ & $\underset{(\mathrm{mg} / \mathrm{kg})}{\mathrm{V}}$ & $\underset{(\mathrm{mg} / \mathrm{kg})}{\mathrm{Zn}}$ & $\begin{array}{c}\mathrm{Zr} \\
(\mathrm{mg} / \mathrm{kg})\end{array}$ \\
\hline 44.22 & 14 & 21 & 1 & 81 & 17 & 165 & 18 & 1 & 8 \\
\hline 44.28 & 14 & 20 & 12 & 1 & 1 & 191 & 11 & 8 & 9 \\
\hline 44.40 & 20 & 16 & 11 & 1 & 31 & 147 & 15 & 11 & 8 \\
\hline 44.28 & 13 & 1 & 1 & 10 & 23 & 161 & 1 & 7 & 9 \\
\hline 44.38 & 13 & 23 & 1 & 11 & 22 & 164 & 1 & 8 & 1 \\
\hline 44.17 & 23 & 1 & 1 & 1 & 14 & 159 & 12 & 1 & 1 \\
\hline 44.36 & 16 & 1 & 1 & 1 & 14 & 147 & 1 & 1 & 1 \\
\hline 43.97 & 14 & 1 & 7 & 17 & 21 & 152 & 16 & 1 & 1 \\
\hline 44.45 & 15 & 1 & 8 & 14 & 22 & 149 & 10 & 1 & 7 \\
\hline 44.39 & 11 & 21 & 1 & 20 & 11 & 145 & 1 & 8 & 8 \\
\hline 44.20 & 11 & 20 & 1 & 25 & 30 & 135 & i & 1 & 1 \\
\hline 44.02 & 12 & 16 & 13 & 1 & 21 & 122 & 1 & 10 & 8 \\
\hline 44.38 & 13 & 20 & 12 & 19 & 23 & 163 & 15 & 23 & 1 \\
\hline 44.43 & 13 & 1 & 1 & 21 & 22 & 193 & 10 & 14 & 8 \\
\hline 44.26 & 1 & 1 & 1 & 14 & 15 & 155 & 1 & 12 & 7 \\
\hline 44.69 & 11 & 26 & 1 & 16 & 5 & 165 & 18 & 1 & 7 \\
\hline 44.85 & 11 & 1 & 1 & 1 & 32 & 241 & 1 & 10 & 8 \\
\hline 44.58 & 24 & 1 & 8 & 20 & 19 & 300 & 13 & 10 & 13 \\
\hline 44.49 & 18 & 16 & 1 & 10 & 19 & 264 & 1 & 8 & 10 \\
\hline 44.61 & 17 & 20 & 11 & 12 & 30 & 249 & 12 & 13 & 1 \\
\hline 42.72 & 14 & 1 & 27 & 13 & 38 & 237 & 46 & 32 & 16 \\
\hline 44.94 & 12 & 1 & 8 & 21 & 19 & 314 & 32 & 16 & 8 \\
\hline 24.26 & 145 & 21 & 151 & 57 & 19 & 52 & 93 & 101 & 123 \\
\hline 44.12 & 12 & 1 & 1 & 17 & 6 & 248 & 1 & 26 & 1 \\
\hline 44.01 & 16 & 1 & 1 & 47 & 20 & 300 & 1 & 16 & 11 \\
\hline 43.13 & 22 & 1 & 15 & 60 & 30 & 450 & 11 & 99 & 15 \\
\hline 44.18 & 15 & 1 & 1 & 44 & 16 & 235 & 1 & 31 & 11 \\
\hline 44.48 & 10 & 21 & 1 & 17 & 15 & 271 & 1 & 18 & 1 \\
\hline 44.32 & 1 & 22 & 1 & 11 & 13 & 249 & 1 & 17 & 8 \\
\hline 44.29 & 15 & 1 & 1 & 123 & 18 & 217 & 1 & 13 & 1 \\
\hline 44.28 & 12 & 1 & 1 & 1 & 22 & 229 & 10 & 8 & 1 \\
\hline 44.14 & 1 & 18 & 1 & 12 & 9 & 241 & 1 & 1 & 7 \\
\hline 44.50 & 1 & 15 & 1 & 24 & 17 & 202 & 1 & 1 & 8 \\
\hline 44.09 & 14 & 32 & 1 & 24 & 22 & 212 & 1 & 1 & 7 \\
\hline 44.99 & 17 & 1 & 1 & 29 & 19 & 373 & 162 & 81 & 14 \\
\hline 43.99 & 24 & 1 & 1 & 18 & 19 & 241 & 1 & 26 & 8 \\
\hline 44.36 & 26 & 24 & 1 & 11 & 18 & 265 & 1 & 20 & 8 \\
\hline 40.01 & 38 & 18 & 12 & 20 & 26 & 369 & 46 & 27 & 65 \\
\hline 43.23 & 21 & 19 & 1 & 15 & 19 & 277 & 13 & 13 & 15 \\
\hline 44.21 & 17 & 25 & 1 & 12 & 1 & 210 & 1 & 10 & 1 \\
\hline 44.24 & 17 & 1 & 1 & 13 & 12 & 202 & 1 & 9 & 1 \\
\hline 44.31 & 15 & 1 & 1 & 26 & 18 & 299 & 11 & 7 & 9 \\
\hline 43.86 & 1 & 1 & 1 & 23 & 20 & 341 & 1 & 1 & 13 \\
\hline 13.75 & 17 & 1 & 1 & 17 & 17 & 92 & 1 & 9 & 1 \\
\hline 43.51 & 13 & 1 & 1 & 16 & 21 & 263 & 10 & 1 & 1 \\
\hline 43.22 & 28 & 1 & 1 & 31 & 28 & 347 & 10 & 24 & 17 \\
\hline 45.63 & 12 & 16 & 14 & 16 & 20 & 145 & 21 & 15 & 1 \\
\hline 45.80 & 15 & 26 & 1 & 29 & 8 & 163 & 1 & 33 & 1 \\
\hline 44.46 & 13 & 18 & 1 & 17 & 11 & 273 & 1 & 15 & 1 \\
\hline 43.73 & 18 & 1 & 10 & 25 & 1 & 199 & 20 & 21 & 14 \\
\hline 44.93 & 13 & 1 & 7 & 21 & i & 152 & 12 & 12 & 11 \\
\hline 45.37 & 18 & 1 & 1 & 25 & 5 & 129 & 44 & 41 & 17 \\
\hline 47.27 & 14 & 1 & 1 & 24 & 15 & 132 & 1 & 20 & 1 \\
\hline 46.92 & 10 & 1 & 1 & 20 & 9 & 138 & 1 & 90 & 1 \\
\hline 44.55 & 13 & 1 & I & 15 & 21 & 259 & 1 & 1 & 8 \\
\hline 46.91 & 16 & 1 & $i$ & 14 & 10 & 140 & 1 & 23 & 1 \\
\hline 44.74 & 11 & 1 & 1 & 1 & 14 & 320 & 1 & 8 & 1 \\
\hline 45.11 & 13 & 1 & 1 & 14 & 18 & 96 & 1 & 1 & 1 \\
\hline 44.33 & 10 & 26 & 8 & 34 & 14 & 330 & 15 & 12 & 12 \\
\hline 43.71 & 19 & 1 & 8 & 37 & 19 & 283 & 13 & 12 & 8 \\
\hline 44.37 & 16 & 1 & 1 & 19 & 15 & 241 & 1 & 9 & 10 \\
\hline 44.43 & 12 & 1 & 8 & 18 & 17 & 256 & 1 & 1 & 1 \\
\hline 44.64 & 14 & 1 & 1 & 24 & 14 & 302 & 19 & 11 & 11 \\
\hline 44.44 & 13 & 1 & 1 & 26 & 23 & 311 & 15 & 1 & 10 \\
\hline 42.39 & 19 & 29 & 1 & 25 & 17 & 293 & 24 & 10 & 23 \\
\hline 44.16 & 15 & 24 & $i$ & 27 & 20 & 246 & 1 & 8 & 1 \\
\hline 42.08 & 21 & 37 & 8 & 25 & 33 & 390 & 14 & 9 & 32 \\
\hline 45.30 & 12 & 1 & 1 & 29 & 26 & 264 & 16 & 14 & 1 \\
\hline 44.63 & 13 & 1 & $i$ & 17 & 6 & 256 & 14 & 11 & 1 \\
\hline 44.76 & 12 & 1 & 10 & 17 & 24 & 351 & 11 & 13 & 12 \\
\hline 44.38 & 13 & 32 & 1 & 34 & 33 & 319 & 1 & 14 & 11 \\
\hline 44.56 & 1 & 19 & 1 & 26 & 27 & 321 & 13 & 11 & 7 \\
\hline 46.42 & 14 & 18 & 1 & 37 & 24 & 159 & 1 & 16 & 1 \\
\hline 46.96 & 11 & 1 & 1 & 19 & 23 & 129 & 1 & 9 & 1 \\
\hline 44.83 & 12 & 28 & 14 & 25 & 17 & 271 & 24 & 26 & 10 \\
\hline 45.15 & 11 & 27 & 1 & 22 & 27 & 290 & 1 & 15 & 1 \\
\hline 46.32 & 11 & 1 & 1 & 14 & 16 & 188 & 16 & 12 & 7 \\
\hline
\end{tabular}


Table 3. Major, minor, and trace elements (XRF) of Sites 867 and 868.

\begin{tabular}{|c|c|c|c|c|c|c|c|c|c|c|c|}
\hline $\begin{array}{l}\text { Core, section, } \\
\text { interval }(\mathrm{cm})\end{array}$ & $\begin{array}{l}\text { Depth } \\
\text { (mbsf) }\end{array}$ & $\begin{array}{l}\mathrm{SiO}_{2} \\
(\%)\end{array}$ & $\begin{array}{l}\mathrm{TiO}_{2} \\
(\%)\end{array}$ & $\begin{array}{c}\mathrm{Al}_{2} \mathrm{O}_{3} \\
(\%)\end{array}$ & $\begin{array}{c}\mathrm{Fe}_{2} \mathrm{O}_{3} \\
(\%)\end{array}$ & $\begin{array}{c}\mathrm{MnO} \\
(\%)\end{array}$ & $\begin{array}{l}\mathrm{MgO} \\
(\%)\end{array}$ & $\begin{array}{c}\mathrm{CaCO}_{3} \\
(\%)\end{array}$ & $\begin{array}{l}\mathrm{P}_{2} \mathrm{O}_{5} \\
(\%)\end{array}$ & $\begin{array}{l}\mathrm{SO}_{3} \\
(\%)\end{array}$ & $\begin{array}{c}\mathrm{Ba} \\
(\mathrm{mg} / \mathrm{kg})\end{array}$ \\
\hline $\begin{array}{l}\text { 143-867B- } \\
\text { IR-1,11-13 }\end{array}$ & 0.11 & 73.41 & 0.011 & 0.12 & 0.08 & 0.011 & 0.07 & 24.15 & 3.48 & 0.25 & 1674 \\
\hline $3 \mathrm{R}-1,54-57$ & 15.64 & 0.27 & 0.016 & 0.15 & 0.08 & 0.006 & 0.28 & 95.86 & 19.54 & 0.96 & 27 \\
\hline $4 \mathrm{R}-1,68-70$ & 18.78 & 1.12 & 0.009 & 0.12 & 0.06 & 0.005 & 0.62 & 94.68 & 0.30 & 0.02 & 75 \\
\hline $6 \mathrm{R}-1,35-37$ & 34.25 & 0.10 & 0.008 & 0.06 & 0.05 & 0.004 & 0.53 & 98.40 & 0.32 & 0.02 & 15 \\
\hline $7 \mathrm{R}-1, \quad 37-39$ & 43.57 & 0.18 & 0.006 & 0.08 & 0.04 & 0.004 & 0.49 & 97.88 & 0.00 & 0.02 & 20 \\
\hline $8 R-2,44-46$ & 54.44 & 0.17 & 0.006 & 0.08 & 0.05 & 0.004 & 0.72 & 97.36 & 0.01 & 0.02 & 1 \\
\hline $9 \mathrm{R}-1,129-132$ & 63.09 & 0.46 & 0.021 & 0.24 & 0.08 & 0.006 & 0.75 & 97.40 & 0.24 & 0.02 & 14 \\
\hline $10 \mathrm{R}-1,111-114$ & 67.51 & 0.28 & 0.007 & 0.08 & 0.05 & 0.005 & 0.78 & 97.02 & 0.06 & 0.02 & 13 \\
\hline $143-868 \mathrm{~A}-$ & & & & & & & & & & & \\
\hline $1 \mathrm{R}-2,58-60$ & 0.11 & 0.28 & 0.027 & 0.16 & 0.08 & 0.006 & 0.69 & 97.59 & 0 & 0.02 & 12 \\
\hline $3 R-1,119-121$ & 15.64 & 0.20 & 0.011 & 0.09 & 0.10 & 0.006 & 0.46 & 97.84 & 0.03 & 0.02 & 13 \\
\hline $4 \mathrm{R}-1,131-133$ & 18.78 & 0.14 & 0.011 & 0.08 & 0.06 & 0.005 & 0.81 & 99.49 & 0.09 & 0.02 & 12 \\
\hline $1 \mathrm{R}-1,87-88$ & 34.25 & 0.05 & 0.005 & 0.06 & 0.04 & 0.005 & 0.93 & 98.09 & 0.04 & 0.02 & 11 \\
\hline
\end{tabular}

Table 4. Microprobe analyses of Sites 865 through 868 .

\begin{tabular}{|c|c|c|c|c|c|}
\hline $\begin{array}{l}\text { Core, section, } \\
\text { interval }(\mathrm{cm})\end{array}$ & Lithology & Component & $\begin{array}{l}\mathrm{CaO} \\
(\%)\end{array}$ & $\begin{array}{l}\mathrm{SrO} \\
(\%)\end{array}$ & $\begin{array}{l}\mathrm{MgO} \\
(\%)\end{array}$ \\
\hline $\begin{array}{l}\text { 143-866A- } \\
74 \mathrm{R}-2,94-97\end{array}$ & Oolite & $\begin{array}{l}\text { Blocky calcite cement } \\
\text { Blocky calcite cement } \\
\text { Blocky calcite cement } \\
\text { Blocky calcite cement } \\
\text { Blocky calcite cement }\end{array}$ & $\begin{array}{l}55.09 \\
55.02 \\
54.54 \\
56.36 \\
56.50\end{array}$ & $\begin{array}{l}0.08 \\
0.00 \\
0.01 \\
0.00 \\
0.03\end{array}$ & $\begin{array}{l}0.98 \\
0.79 \\
0.86 \\
0.61 \\
1.41\end{array}$ \\
\hline $\begin{array}{l}867 \mathrm{~B}- \\
\text { 1R-3, 23-24 }\end{array}$ & Rudstone & $\begin{array}{l}\text { Blocky calcite cement } \\
\text { Blocky calcite cement } \\
\text { Blocky calcite cement } \\
\text { Blocky calcite cement } \\
\text { Scalenohedral cement } \\
\text { Scalenohedral cement } \\
\text { Scalenohedral cement }\end{array}$ & $\begin{array}{l}63.83 \\
58.07 \\
62.42 \\
55.05 \\
53.57 \\
55.74 \\
61.88\end{array}$ & $\begin{array}{l}0.04 \\
0.05 \\
0.00 \\
0.00 \\
0.07 \\
0.00 \\
0.10\end{array}$ & $\begin{array}{l}1.52 \\
1.99 \\
1.69 \\
0.98 \\
0.76 \\
1.82 \\
2.09\end{array}$ \\
\hline $\begin{array}{l}865 \mathrm{~A}- \\
73 \mathrm{R}-1,92-94\end{array}$ & Packstone/grainstone & $\begin{array}{l}\text { Blocky calcite cement } \\
\text { Blocky calcite cement } \\
\text { Blocky calcite cement } \\
\text { Blocky calcite cement } \\
\text { Blocky calcite cement }\end{array}$ & $\begin{array}{l}55.65 \\
57.76 \\
56.72 \\
59.70 \\
57.50\end{array}$ & $\begin{array}{l}0.02 \\
0.01 \\
0.00 \\
0.01 \\
0.02\end{array}$ & $\begin{array}{l}1.37 \\
1.30 \\
0.93 \\
1.13 \\
1.55\end{array}$ \\
\hline $\begin{array}{l}\text { 866A- } \\
164 \mathrm{R}-2,140-14\end{array}$ & & $\begin{array}{l}\text { Ooid core } \\
\text { Ooid core } \\
\text { Ooid core } \\
\text { Ooid margin } \\
\text { Ooid margin } \\
\text { Ooid margin } \\
\text { Blocky calcite cement } \\
\text { Blocky calcite cement } \\
\text { Blocky calcite cement }\end{array}$ & $\begin{array}{l}56.81 \\
59.48 \\
58.11 \\
55.66 \\
53.47 \\
50.35 \\
38.42 \\
36.46 \\
35.82\end{array}$ & $\begin{array}{l}0.06 \\
0.08 \\
0.07 \\
0.06 \\
0.05 \\
0.02 \\
0.01 \\
0.00 \\
0.00\end{array}$ & $\begin{array}{r}1.80 \\
1.97 \\
1.84 \\
1.57 \\
1.51 \\
1.35 \\
20.15 \\
19.71 \\
18.82\end{array}$ \\
\hline $\begin{array}{l}866 \mathrm{~A}- \\
129 \mathrm{R}-1,124-128\end{array}$ & Oolite & $\begin{array}{l}\text { Ooid core } \\
\text { Ooid core } \\
\text { Ooid core } \\
\text { Ooid core } \\
\text { Ooid core } \\
\text { Blocky calcite cement } \\
\text { Blocky calcite cement } \\
\text { Blocky calcite cement }\end{array}$ & $\begin{array}{l}53.87 \\
51.58 \\
42.14 \\
48.90 \\
49.32 \\
55.14 \\
53.76 \\
53.09\end{array}$ & $\begin{array}{l}0.03 \\
0.08 \\
0.00 \\
0.06 \\
0.09 \\
0.01 \\
0.00 \\
0.01\end{array}$ & $\begin{array}{l}1.53 \\
1.36 \\
1.05 \\
1.55 \\
1.59 \\
0.90 \\
1.49 \\
1.32\end{array}$ \\
\hline $\begin{array}{l}865 \mathrm{~A}- \\
28 \mathrm{R}-1,26-27\end{array}$ & Wackestone/packstone & $\begin{array}{l}\text { Blocky calcite cement } \\
\text { Blocky calcite cement } \\
\text { Blocky calcite cement } \\
\text { Shell } \\
\text { Shell } \\
\text { Shell }\end{array}$ & $\begin{array}{l}56.58 \\
55.41 \\
56.76 \\
58.88 \\
54.62 \\
55.81\end{array}$ & $\begin{array}{l}0.00 \\
0.00 \\
0.01 \\
0.19 \\
0.14 \\
0.14\end{array}$ & $\begin{array}{l}1.07 \\
0.97 \\
1.15 \\
0.93 \\
4.08 \\
0.71\end{array}$ \\
\hline $\begin{array}{l}868 \mathrm{~A}- \\
4 \mathrm{R}-2,33-35\end{array}$ & Grainstone/rudstone & Peloid & 57.92 & 0.02 & 0.00 \\
\hline
\end{tabular}

\section{Site 868 (Resolution Guyot)}

Site 868 is characterized by high carbonate contents (96\% to $98 \%$ $\mathrm{CaCO}_{3}$, Table 3). Phosphatization plays only a minor part in the investigated samples: Sample 143-868A-4R-1, 119-121 cm (18.78 mbsf) consists of up to $0.09 \% \mathrm{P}_{2} \mathrm{O}_{5}$. With increasing distance from the sediment/water interface, the $\mathrm{SiO}_{2}, \mathrm{Al}_{2} \mathrm{O}_{3}, \mathrm{TiO}_{2}, \mathrm{MnO}$, and $\mathrm{Cu}$ values decrease continuously downward (Table 3 ).

\section{Cement Geochemistry}

Microprobe Analyses

Selected samples from Sites 865 to 868 have been analyzed for their $\mathrm{Ca}, \mathrm{Mg}$, and $\mathrm{Sr}$ contents of blocky calcite cement (Table 4). Where possible, scalenohedral cements, shells or different ooid layers were separated and analyzed. Strontium contents are mainly below the detection limit for this method. 
Table 3 (continued).

\begin{tabular}{ccccccc}
\hline $\begin{array}{c}\mathrm{Ce} \\
(\mathrm{mg} / \mathrm{kg})\end{array}$ & $\begin{array}{c}\mathrm{Cr} \\
(\mathrm{mg} / \mathrm{kg})\end{array}$ & $\begin{array}{c}\mathrm{Cu} \\
(\mathrm{mg} / \mathrm{kg})\end{array}$ & $\begin{array}{c}\mathrm{La} \\
(\mathrm{mg} / \mathrm{kg})\end{array}$ & $\begin{array}{c}\mathrm{Sr} \\
(\mathrm{mg} / \mathrm{kg})\end{array}$ & $\begin{array}{c}\mathrm{Zn} \\
(\mathrm{mg} / \mathrm{kg})\end{array}$ & $\begin{array}{c}\mathrm{Zr} \\
(\mathrm{mg} / \mathrm{kg})\end{array}$ \\
\hline 1 & 7 & 15 & 20 & 286 & 12 & 1 \\
33 & 1 & 17 & 20 & 1057 & 28 & 9 \\
22 & 9 & 14 & 19 & 332 & 27 & 33 \\
24 & 1 & 1 & 20 & 177 & 12 & 1 \\
19 & 1 & 1 & 21 & 445 & 9 & 1 \\
20 & 1 & 18 & 23 & 216 & 8 & 1 \\
1 & 11 & 18 & 21 & 286 & 1 & 1 \\
1 & 1 & 1 & 22 & 251 & 8 & 1 \\
& & & & & & \\
1 & 1 & 23 & 5 & 260 & 1 & 1 \\
1 & 1 & 21 & 21 & 845 & 8 & 1 \\
1 & 8 & 1 & 22 & 243 & 1 & 1 \\
& 1 & 1 & 21 & 263 & 1 & 1 \\
\hline
\end{tabular}

All of the cement samples are of low-Mg calcite (blocky cements consist of $0.37 \%$ to $0.59 \% \mathrm{Mg}$, and the scalenohedral cement in Sample 143-867B-1R-3, 23-24 cm shows values between $1.09 \%$ and $1.26 \% \mathrm{Mg}$, Table 4). The gastropod shell in Sample 143-865A-28R-1, 26-27 cm, consists of up to $2.46 \% \mathrm{Mg}$ of pronounced higher contents. Ooids of Sample 143-866A-129R-1, 124-128 cm, exhibit decreasing $\mathrm{Mg}$ and $\mathrm{Ca}$ contents from the center to the margin of the ooid (Fig. 7), which is probably related to leaching that preceding the dolomitization of pore spaces. The blocky, pore-filling cement is of dolomite mineralogy (Fig. 7) and consists of $11.35 \%$ to $12.15 \% \mathrm{Mg}$.

\section{AA/ICP Analyses}

Twenty samples of blocky calcite cement, shells, dolomite crystals (rhombs), fine-grained dolomite, and oncoids were analyzed for their $\mathrm{Ca}, \mathrm{Mg}, \mathrm{Sr}, \mathrm{Mn}, \mathrm{Fe}$, and $\mathrm{Zn}$ contents (Table 5). The samples of calcite cement exhibit contents of $33.3 \%$ to $40.0 \% \mathrm{Ca}$, the shells have values of about $38.8 \%$, and the blocky cements values are about $37.8 \% \mathrm{Ca}$. The dolomite samples consist of $26.3 \% \mathrm{Ca}$. The $\mathrm{Mg}$ values vary between $0.13 \%$ and $0.33 \%$ in the calcite samples, while in the dolomite samples, between $8.73 \%$ and $11.1 \% \mathrm{Mg}$. The $\mathrm{Sr}$ contents are lowest in the blocky cement samples of Site 866, while we obtained values of $151 \mathrm{mg} / \mathrm{kg} \mathrm{Sr}$ for Site 865 . Oncoids consist of $334 \mathrm{mg} / \mathrm{kg}$ $\mathrm{Sr}$, dolomite cement of $156 \mathrm{mg} / \mathrm{kg} \mathrm{Sr}$ (mean). Mollusk shells contain 838 to $973 \mathrm{mg} / \mathrm{kg} \mathrm{Sr}$. These values are within the range of values analyzed by Maliva and Dickson (1992) for different bivalves of the Purbeck Formation of southern England and are related to the primary aragonite mineralogy.

The $\mathrm{Mn}$ and Fe contents (Fig. 8) are low in Hole 866A (Table 5), but relatively high in the lower third of Hole $865 \mathrm{~A}$ (Unit IV, see Table 5). The distributions of both $\mathrm{Fe}$ and $\mathrm{Mn}$ and especially the $\mathrm{Mn} / \mathrm{Fe}$ ratios (10 to 42 at Site $865,<1$ to 13 at Site 866), are the reason that the samples of Site 865 Unit IV show cathodoluminescence, while those of Site 866 do not (cf. Hemming et al., 1989). The dolomite samples have low Mn values ( 2 to $44 \mathrm{mg} / \mathrm{kg}$ ), but Fe values of up to $3172 \mathrm{mg} / \mathrm{kg}$. $\mathrm{Zn}$ contents are relatively high in the dolomites (10 to $15 \mathrm{mg} / \mathrm{kg}$ ) and oncoids ( 9 to $12 \mathrm{mg} / \mathrm{kg}$ ), which may be interpreted as having originated under hypersaline conditions, in which $\mathrm{Zn}$ is more readily incorporated into the calcite/dolomite crystal lattice (cf. Thein, 1985; Farr, 1992). Shells and blocky calcite cements contain only minor amounts of $\mathrm{Zn}$.

\section{Stable Isotope Analyses $\left(\delta^{18} \mathrm{O} /{ }^{13} \mathrm{C}\right)$}

Although the morphology and texture of the analyzed cement samples indicate a meteoric-phreatic origin (see chapter on cements), the $\delta^{13} \mathrm{C}$ values that we found are not negative, as expected (Hudson, 1977), but range from +1.1 to +3.4 (Fig. 9). An explanation of positive $\delta^{13} \mathrm{C}$ values for cements of freshwater origin would be a nonvegetated island and where soil gas would be absent as the source for carbon (cf. Winterer et al., this volume).
The $\delta^{18} \mathrm{O}$ values are mainly negative and range between +1.5 and -5.9. The most negative values indicate a trend toward meteoric cement composition.

However, the data plotted in Figure 9 could not be interpreted as definitive marine or freshwater signals. Comparison with published data (Algeo et al., 1992; Anderson and Arthur, 1983; Dickson and Coleman, 1980; Hudson, 1977; Nesse et al., 1980; Quinn and Matthews, 1990; Saller and Moore, 1989) shows that some samples suggest a marine-phreatic origin, and one sample would imply burial diagenesis. Most of the samples are located on a path from marinephreatic to burial. This leads us to conclude that many originally freshwater cements may have been overprinted in a marine or shallow burial diagenetic environment. However, no petrographic evidence, such as marine cements growing on top of freshwater cements, could be found.

\section{Chemistry/Microfacies Relationships}

Petrographical data, including components, matrix, cements, and porosity in samples from all sites (Figs. 2 and 3), were used to compare geochemical compositions with the lithofacies.

The component-, porosity- and cement-rich grainstones and rudstones show the highest $\mathrm{MgO}$ contents (Fig. 10A), which result from the close relationship of most of these limestones with the dolomitized unit in the lower part of Site 866. The highest $\mathrm{CaCO}_{3}$ contents were found within the floatstones, grainstones, and wackestones to packstones, and the lowest are in the grainstones to rudstones and rudstones of the lower part of Site 866 (compare with $\mathrm{MgO}$ in Fig. 10A). $\mathrm{SiO}_{2}, \mathrm{Al}_{2} \mathrm{O}_{3}, \mathrm{Fe}_{2} \mathrm{O}_{3}, \mathrm{Zr}$, and $\mathrm{V}$ contents are more common in mud-supported lithologies and represent the clayey portion of these facies types (Fig. 10B). The distributions of $\mathrm{MnO}, \mathrm{Ba}$, and phosphate demonstrate that these elements, respective compounds, are enriched during a later diagenetic/epigenetic phase: precipitation of a manganese crust and some barite on top of the shallow-water sections and the penetrative phosphatization in the upper portion of the platform carbonates. The instability of $\mathrm{Sr}$, which is highly mobile (Morse and Mackenzie, 1990), is higher in samples having relatively high percolation rates from increased porosity and/or permeability. Therefore, $\mathrm{Sr}$ values (Fig. 10A) are lower in the grain-supported lithologies. $\mathrm{SO}_{3}$ shows maximum values in wackestones and mudstones (Fig. 10A), which is probably related to a greater sealing effect in the muddier limestones. The good correlation of $\mathrm{SO}_{3}$ with TOC (cf. Baudin et al., this volume) in distinct layers confirms this interpretation.

Figure 11 shows a cluster analysis in R-mode by a weighted-pergroup method (program MEKO; A. Siehl, unpubl. data). A clear differentiation is seen between two main groups: variables that reflect the calcareous part of the samples on one side, and elements responsible for the terrigenous portion on the other side. $\mathrm{CaCO}_{3}$ and $\mathrm{MgO}$ group with most of the variables obtained from thin-section descriptions except for "matrix," "bioturbation," and "sponge spicules." These descriptions are included within the terrigenous group and are typical for the muddier (more clayey) limestones. Within the calcareous group, variables that are related to calcite or dolomite are forming subunits: the amount of components and of porosity show good correlations with the $\mathrm{CaCO}_{3}$ content, dolomite content, and the depth below seafloor with $\mathrm{MgO}$. The terrigenous portion in Figure 11 is subdivided into metals and oxides, and the metals show the best correlations, especially the elements that are related to the manganese crusts $(\mathrm{Mn}, \mathrm{Cu}, \mathrm{Zn}, \mathrm{Ba})$. Strontium and phosphate cluster because their distribution is related to diagenetic alteration.

\section{CONCLUSIONS}

This study indicates that distinct geochemical trends and diagenetic features allow for the reconstruction of primary mineralogical composition and early to late diagenetic alterations in relation to sedimentary sequences. 

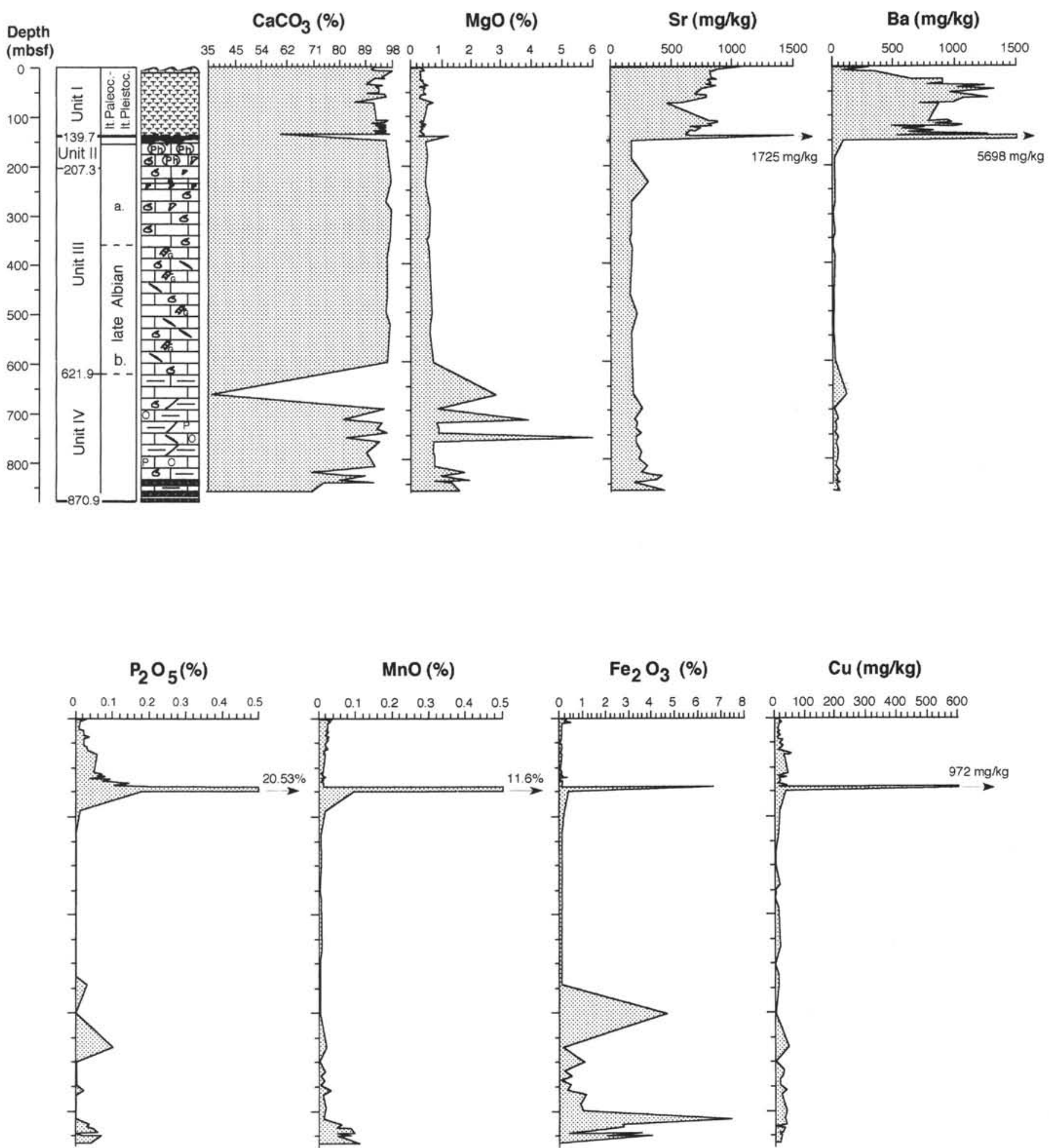

Figure 4. $\mathrm{CaCO}_{3}, \mathrm{MgO}, \mathrm{Sr}, \mathrm{Ba}, \mathrm{P}_{2} \mathrm{O}_{5}, \mathrm{MnO}, \mathrm{Fe}_{2} \mathrm{O}_{3}$, and $\mathrm{Cu}$ contents in Hole $865 \mathrm{~A}$ (Allison Guyot). Note the dramatical change in $\mathrm{Ba}$ and $\mathrm{Sr}$ contents between the Tertiary pelagic cap nannofossil/foraminifer oozes and the Cretaceous shallow-water limestones, and significant penetrative phosphatization and manganese mineralization, including associated metals.

\section{Cementation and Porosity Development}

The generally low recovery implies that a relatively larger portion of the guyot limestones is less lithified. Recovery increased downward, which on one side is related to the facies, on the other side to higher porosity in the upper part of the drill holes. However, generally uncemented layers may be present repeatedly throughout the sections. Allison and Resolution Guyot Cretaceous limestones were exposed to percolating meteoric waters at various times during their history. Because they were deposited mostly in shallow water, small changes in sea level resulted in subaerial exposure early in their history (see also Enos, 1985). Minor surficial exposure might result 


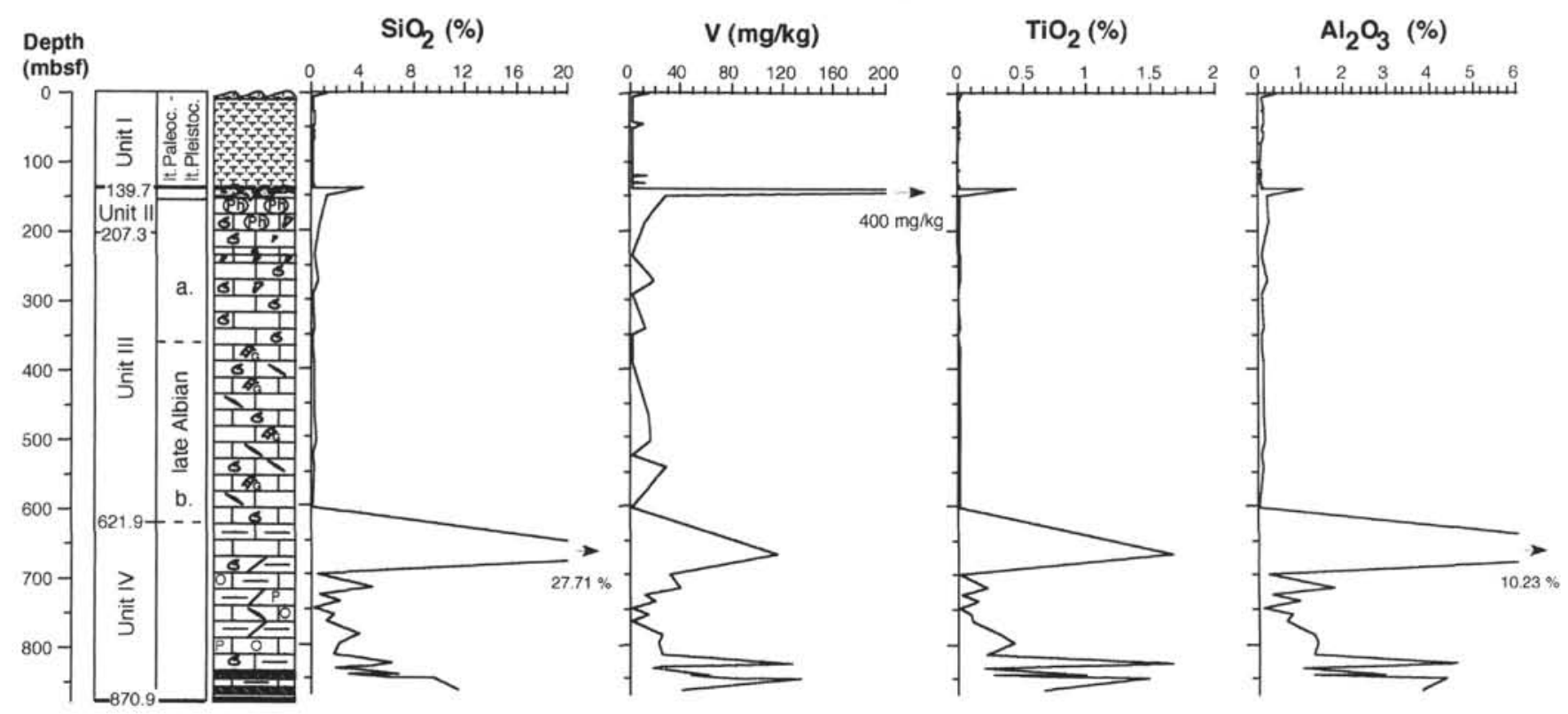

Figure 5. The distribution of the elements $\mathrm{SiO}_{2}, \mathrm{~V}, \mathrm{TiO}_{2}$, and $\mathrm{Al}_{2} \mathrm{O}_{3}$ can be used as a proxy for the clay content of limestone samples (Hole 865A, Allison Guyot).

in relatively deep percolation of freshwater and creation of accompanying cement and porosity types, and earlier stages of diagenesis may have been obliterated. As the rocks are now in the marine environment, some of the chemical signals currently present may be the result of later superimposing.

\section{Distribution of Major, Minor, and Trace Elements}

The uphole decreasing clayey fraction in these sediments documents directly the subsidence of the volcanic island, which, at one stage, was completely overgrown by the carbonate bank. The distribution of minor and trace elements, which are related to terrigenous influx reflects a cyclical pattern, which is probably related to lowamplitude sea-level fluctuations. Cyclicity of chemical data in Site 866 shows a high correlation with sequence stratigraphic interpretations. Low Mn concentrations explain the nonluminescence of most of the samples. Phosphatized levels contain between $0.01 \%$ and $20 \%$ $\mathrm{P}_{2} \mathrm{O}_{5}$. We can distinguish between two main processes responsible for phosphate enrichment: on one hand, we identified syngenetic phosphate on maximum flooding surfaces; and on the other hand, more obvious phosphate enrichment occurred on the platform tops by late diagenetic phosphatization (Hein et al., 1993). $\mathrm{SO}_{3}$ and $\mathrm{V}$ show good correlations with the organic matter and are therefore related to more restricted depositional conditions.

\section{Isotopic Trends}

Oxygen and carbon isotopic compositions of different cement samples show gradual enrichment with depth (cf. Zempolich, 1989; Site 866: compare also with Jenkyns, this volume), but no clear meteoric or marine signal was found. However, a distinct diagenetic trend emerges: most of the data are located on a path from the meteoric to the marine values of published data. This may be explained by marine overprinting of the freshwater-cemented limestones during development from an emerged carbonate bank toward a submarine plateau. Similar observations have been made at other drowned shallow-water carbonate plateaus (e.g., Wombat Plateau, off Northwest Australia, cf. Röhl et al., 1992).

\section{Facies Dependence of Diagenetic Alterations and the Diagenetic Model}

Although the Cretaceous open-marine carbonate banks show structural and morphological differences in comparison to modern atolls (Fig. 12), distinct facies types or small-scale sequences exhibit common associations of diagenetic alterations typically found in shallowwater carbonates. The recovered material may represent well-cemented diagenetic caps of the sequences, whereas the nonrecovered portions probably are less cemented and highly porous. This interpretation agrees with the model of large amounts of seawater flushing through the upper part of the shallow-water limestones, as proposed by Paull et al. (this volume). Diagenesis commenced soon after deposition. Differences in primary composition, microstructure, sorting, and packing in combination with the changing influence of meteoric and marine waters resulted in a complex diagenetic development. The guyot limestones have been in an unconfined aquifer with diffuse flow throughout most of their history. The diagenetic development of the carbonates can be divided into two main phases: (1) an early phase was dominated by pore waters that percolated through the sediment pile, while (2) the carbonates were influenced alternatively by seawater and/or freshwater. Diagenesis in this situation shows the effects of both marinephreatic and meteoric-phreatic to meteoric-vadose environments. In the course of this development, the limestones were affected by neomorphism, replacement, dissolution, cementation, and fracturing. In a later phase, subaerial exposure and karstification development (cf. Winterer et al., this volume) overprinted the earlier diagenetic stages in the upper portion of the sections, before the carbonate edifices subsided and drowned.

All observations of diagenetic features are combined to form a diagenetic model shown in Figure 13, which summarizes the stages recognized in different types of small-scale sequences defined by Strasser et al. (this volume).

\section{ACKNOWLEDGMENTS}

The authors are grateful to the Ocean Drilling Program for inviting us to participate on Leg 143, to all members of the Shipboard 
A

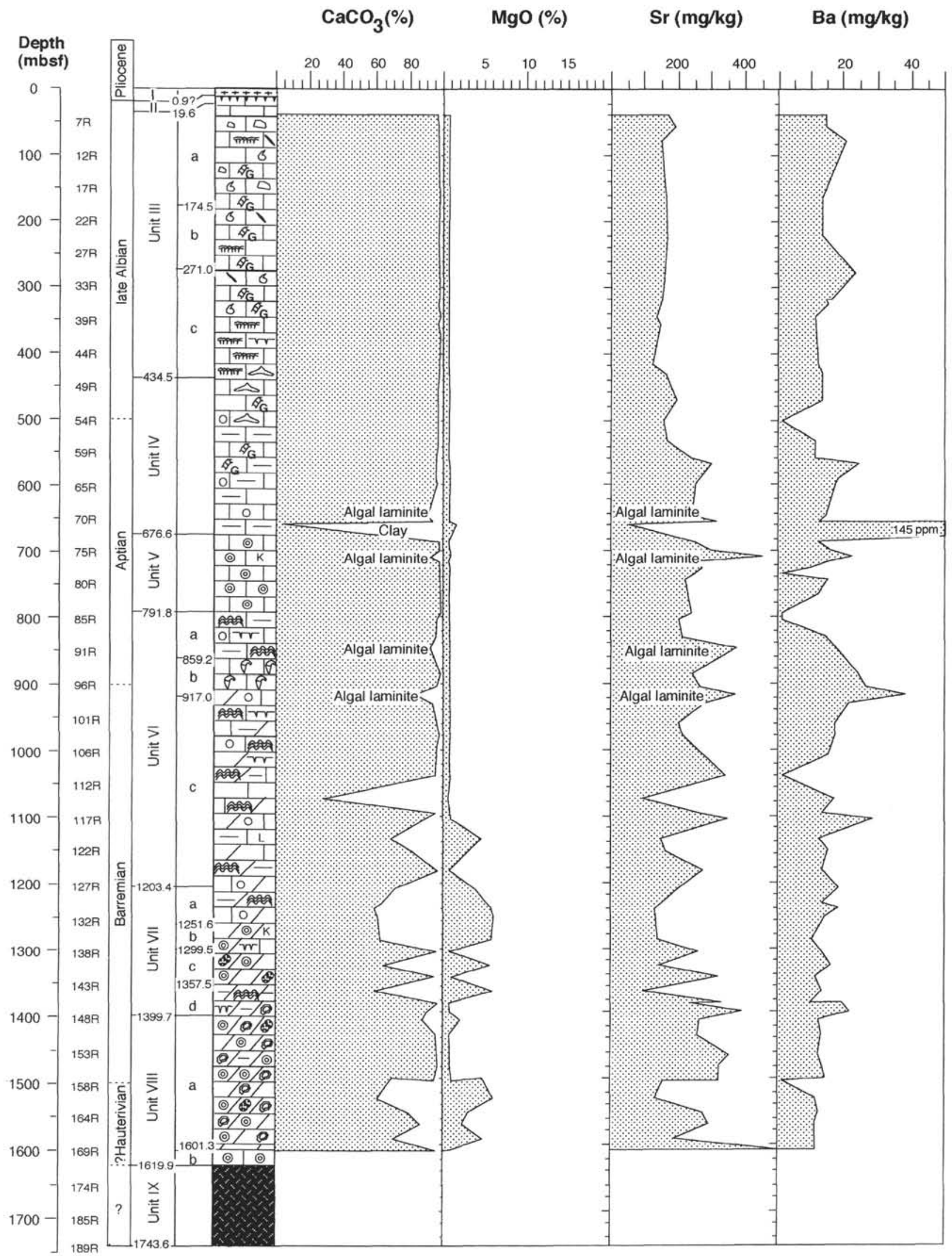

Figure 6. A. $\mathrm{CaCO}_{3}, \mathrm{MgO}, \mathrm{Sr}$, and Ba contents in Hole 866 A (Resolution Guyot). Incisions in the carbonate distribution result from algal laminite or clay layers and, in the lower portion, are caused by dolomitized limestones or dolomites. $\mathrm{B} . \mathrm{P}_{2} \mathrm{O}_{5}, \mathrm{Al}_{2} \mathrm{O}_{3}, \mathrm{SiO}_{2}$, and $\mathrm{MnO}$ contents in Hole 866 A (Resolution Guyot). Because of fewer samples, the penetrative phosphatization is not as clearly visible as in Hole 865A, but several levels with phosphate peaks occur. Arrows indicate a cyclicity of $\mathrm{MnO}$ distribution. Peaks of $\mathrm{Al}_{2} \mathrm{O}_{3}$ and $\mathrm{SiO}_{2}$ in the same level reflect clay contents, single $\mathrm{SiO}_{2}$ peaks are possibly volcanic ash. $\mathrm{C} . \mathrm{SO}_{3}, \mathrm{~V}, \mathrm{Cu}$, and $\mathrm{Zn}$ contents in Hole 866A (Resolution Guyot). Arrows indicate a cyclicity of both $\mathrm{Cu}$ and $\mathrm{Zn}$. Peaks of $\mathrm{SO}_{3}$, in some cases also V, reflect the distribution of organic material (for further explanation see text). 


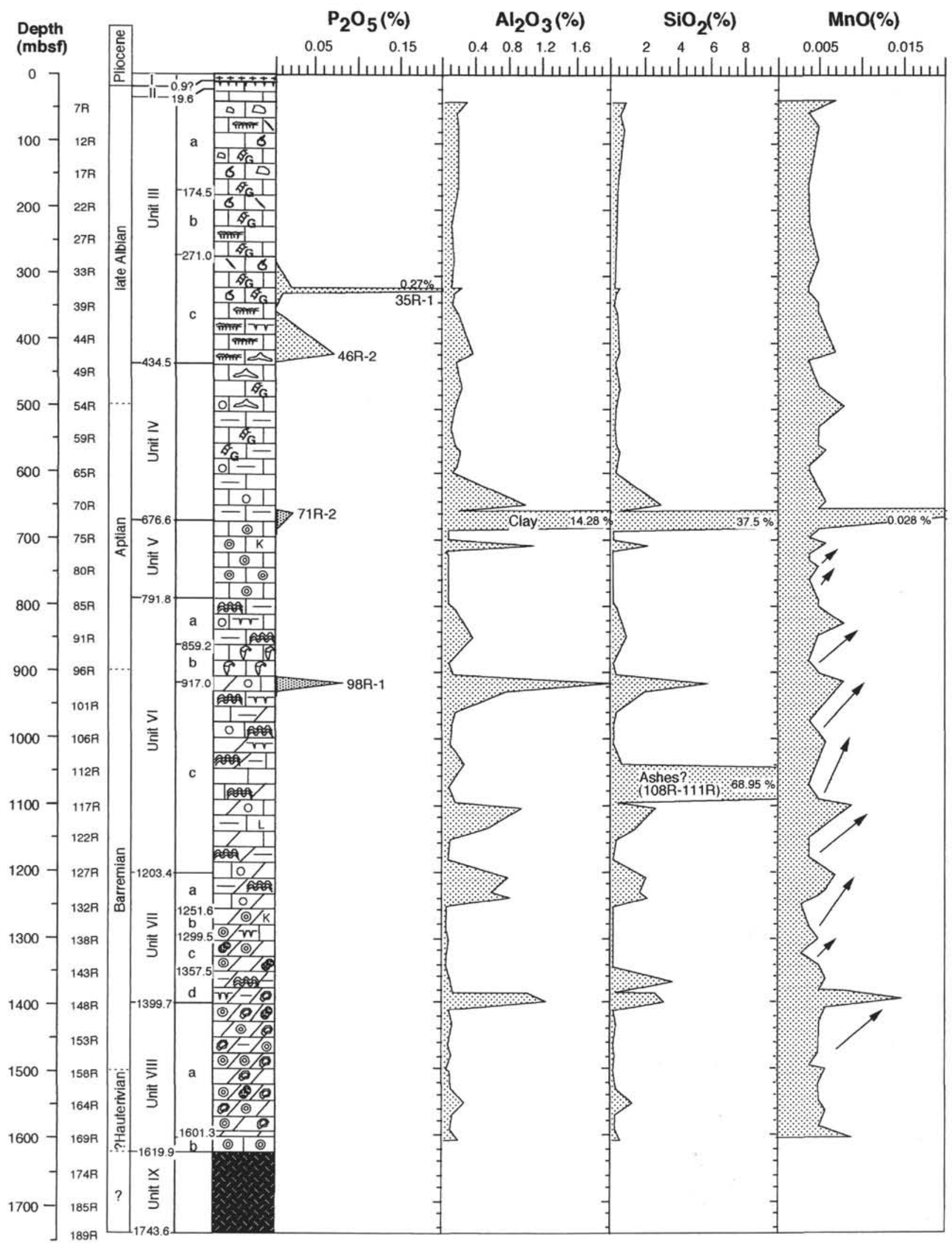

Figure 6 (continued). 
C



Figure 6 (continued). 
Table 5. ICP/AA analyses of Sites 865 to 868 .

\begin{tabular}{|c|c|c|c|c|c|c|c|c|}
\hline $\begin{array}{l}\text { Core, section, } \\
\text { interval }(\mathrm{cm})\end{array}$ & Component & $\begin{array}{l}\text { Depth } \\
\text { (mbsf) }\end{array}$ & $\begin{array}{c}\mathrm{Mn} \\
(\mathrm{mg} / \mathrm{kg})\end{array}$ & $\begin{array}{l}\mathrm{Ca} \\
(\%)\end{array}$ & $\begin{array}{l}\mathrm{Mg} \\
(\%)\end{array}$ & $\begin{array}{c}\mathrm{Fe} \\
(\mathrm{mg} / \mathrm{kg})\end{array}$ & $\begin{array}{c}\mathrm{Sr} \\
(\mathrm{mg} / \mathrm{kg})\end{array}$ & $\begin{array}{c}\mathrm{Zn} \\
(\mathrm{mg} / \mathrm{kg})\end{array}$ \\
\hline \multicolumn{9}{|l|}{ 143-865A- } \\
\hline $39 \mathrm{R}-\mathrm{CC}, 28-29$ & Brown patches & 342.18 & 15 & 386 & 3.5 & 392 & 170 & 2.5 \\
\hline $87 \mathrm{R}-2,100-103$ & Cem. pelec. mold & 807.81 & 118 & 381 & 4.1 & 1,614 & 158 & 1.3 \\
\hline $89 R-1,46-48$ & Big shell, cem. & 825.26 & 394 & 400 & 3.4 & 3,938 & 117 & 4.1 \\
\hline $89 R-4,42-45$ & Cem. pelec. mold & 829.34 & 506 & 353 & 6.5 & 10,390 & 243 & 3.3 \\
\hline $91 R-4,67-71$ & Cem. pelec. mold & 846.03 & 2100 & 379 & 2.2 & 801 & 87 & 1.0 \\
\hline $94 \mathrm{R}-3,129-131$ & Elongated shells & 867.19 & 133 & 381 & 1.3 & 891 & 838 & 1.8 \\
\hline \multicolumn{9}{|l|}{ 143-867B- } \\
\hline $1 \mathrm{R}-1,11-13$ & Cem. pelec, mold & 0.11 & 3254 & 379 & 3.1 & 152 & 551 & 35.8 \\
\hline $2 \mathrm{R}-2,28-30$ & Cem. pelec. mold & 9.88 & 12 & 386 & 3.7 & 207 & 260 & 5.0 \\
\hline 7R-1, 37-39 & Cem. pelec. mold & 43.57 & 9 & 333 & 2.8 & 60 & 188 & 1.7 \\
\hline $8 R-2,44-46$ & Cem. pelec. mold & 54.44 & 4 & 360 & 2.7 & 122 & 72 & 18.3 \\
\hline \multicolumn{8}{|l|}{ 143-868A- } & 1.6 \\
\hline \multicolumn{9}{|l|}{$143-866 \mathrm{~A}-$} \\
\hline 8R-CC, 3-5 & Cem. shell & 57.33 & 8 & 395 & 3.5 & 104 & 263 & 4.8 \\
\hline $46 \mathrm{R}-1,108-110$ & Cem. pelec. mold & 416.38 & 1 & 381 & 3.1 & 29 & 72 & 20.3 \\
\hline $88 \mathrm{R}-1,22-25$ & Cem. pelec. mold & 821.12 & 18 & 383 & 3.7 & 143 & 129 & 1.5 \\
\hline $103 R-1,60-62$ & Cem. pelec. mold & 963.00 & 11 & 383 & 2.8 & 13 & 56 & 1.0 \\
\hline $105 \mathrm{R}-1,82-84$ & Cem. pelec. mold & 982.12 & 11 & 398 & 3.3 & 9 & 77 & 2.2 \\
\hline $133 \mathrm{R}-1,129-121$ & Dolomite rhombs & 1252.79 & 17 & 238 & 111.0 & 87 & 135 & 14.2 \\
\hline 134R-2, 26-28 & Dolomite rhombs & 1262.96 & 5 & 237 & 111.0 & 155 & 125 & 15.0 \\
\hline $146 \mathrm{R}-2,33-35$ & Cem. pelec. mold & 1378.23 & 24 & 386 & 4.2 & 24 & 58 & 11.7 \\
\hline $159 \mathrm{R}-1,87-89$ & Oncoids & 1501.77 & 10 & 346 & 31.0 & 61 & 286 & 12.1 \\
\hline $159 \mathrm{R}-1,87-89$ & Dolom. matrix & 1501.77 & 15 & 265 & 90.5 & 70 & 132 & 9.9 \\
\hline $168 R-3,78-79$ & Oncoids & 1593.82 & 28 & 369 & 8.0 & 388 & 368 & 8.8 \\
\hline $168 R-3,78-79$ & Dolom. matrix & 1593.82 & 2 & 318 & 8.9 & 2,055 & 239 & 10.1 \\
\hline $163 R-1,75-78$ & Oncoids & 1541.05 & 9 & 370 & 11.8 & 163 & 348 & 2.7 \\
\hline 163R-1,75-78 & Dolom. matrix & 1541.05 & 44 & 259 & 87.3 & 3,172 & 151 & 2.0 \\
\hline
\end{tabular}

Note: For further explanation, see text.

Table 6. Stable isotope analyses.

\begin{tabular}{|c|c|c|}
\hline $\begin{array}{l}\text { Core, section, } \\
\text { interval }(\mathrm{cm})\end{array}$ & $\delta^{13} \mathrm{C}$ & $8^{18} \mathrm{O}$ \\
\hline $\begin{array}{l}143-865 \mathrm{~A}- \\
20 \mathrm{R}-1,26-27 \\
89 \mathrm{R}-1,46-48\end{array}$ & $\begin{array}{l}2.77 \\
3.27\end{array}$ & $\begin{array}{l}-1.51 \\
-5.90\end{array}$ \\
\hline $\begin{array}{l}143-866 \mathrm{~A}- \\
46 \mathrm{R}-1,108-110 \\
62 \mathrm{R}-1,53-55 \\
92 \mathrm{R}-1,20-21 \\
97 \mathrm{R}-1,16-18 \\
103 \mathrm{R}-1,60-62 \\
105 \mathrm{R}-1,82-84 \\
106 \mathrm{R}-1,42-43 \\
108 \mathrm{R}-1,55-57 \\
118 \mathrm{R}-1,104-106 \\
119 \mathrm{R}-1,34-35 \\
125 \mathrm{R}-1,2-4 \\
127 \mathrm{R}-1,31-33\end{array}$ & $\begin{array}{l}2.83 \\
2.50 \\
2.49 \\
3.44 \\
1.79 \\
2.25 \\
2.40 \\
2.00 \\
1.13 \\
1.83 \\
2.05 \\
1.96\end{array}$ & $\begin{array}{r}1.51 \\
-2.16 \\
-3.37 \\
-2.11 \\
-2.19 \\
-2.69 \\
-2.75 \\
-2.84 \\
-3.35 \\
-2.87 \\
-3.21 \\
-3.30\end{array}$ \\
\hline $\begin{array}{l}143-867 \mathrm{~B}- \\
1 \mathrm{R}-3,23-24 \\
8 \mathrm{R}-2,44-46 \\
9 \mathrm{R}-2,24-27 \\
3 \mathrm{R}-1,119-121 \\
3 \mathrm{R}-1,119-121 \\
4 \mathrm{R}-2,33-35\end{array}$ & $\begin{array}{l}2.96 \\
3.02 \\
2.60 \\
2.39 \\
2.01 \\
2.68\end{array}$ & $\begin{array}{r}-1.84 \\
0.07 \\
-0.89 \\
-3.02 \\
-4.55 \\
-1.05\end{array}$ \\
\hline
\end{tabular}

Scientific Party of Leg 143 for their excellent cooperation on board the ship, and the free exchange of data after the cruise, and to the ODP personnel and the SEDCO crew, who made Leg 143 a success. U.R. acknowledges financial support by the Deutsche Forschungsgemeinschaft. A.S. was financially supported by the Swiss National Science Foundation. J. Lodziak and D. Requard, Hannover, provided XRF analyses; H. Rösch, Hannover, XRD analyses; M. Geyh and P. Posimowski, Hannover, analyses of stable isotopes; and O. Elsholz, Hannover, ICP/AA analyses. A. Bruns, Hannover, and D. Cao, Fribourg, helped with the SEM analysis, and G. Hartmann, Göttingen, with microprobe analyses. We are grateful for critical comments by L. Land, J. Sinton, and an unknown reviewer.

\section{REFERENCES}

Aissaoui, D.M., 1988. Magnesian calcite cements and their diagenesis: dissolution and dolomitization, Mururoa Atoll. Sedimentology, 35:821-841.

Algeo, T.J., Wilkinson, B.H., and Lohmann, K.C., 1992. Meteoric-burial diagenesis of middle Pennsylvanian limestones in the Orogrande Basin, New Mexico: water/rock interactions and basin geothermics. J. Sediment. Petrol., 62:652-670.

Anderson, T.F., and Arthur, M.A., 1983. Stable isotopes of oxygen and carbon and their application to sedimentologic and paleoenvironmental problems. SEPM Short Course, 10:1-151.

Budd, D.A., 1989. Micro-rhombic calcite and microporosity in limestones: a geochemical study of the Lower Cretaceous Thamama Group. Sediment. Geol., 63:293-311.

Chafetz, H.S., 1986. Marine peloids: a product of bacterially induced precipitation of calcite. J. Sediment. Petrol., 56:812-817.

Choquette, P.W., and Pray, L.C., 1970. Geologic nomenclature and classification of porosity in sedimentary carbonates. AAPG Bull., 54:207-244.

Dickson, J.A.D., and Coleman, M.L., 1980. Changes in carbon and oxygen isotope composition during limestone diagenesis. Sedimentology, 27:107118.

Enos, P., 1985. Diagenetic evolution of Cretaceous reefs in Mexico. Proc. 5th Int. Coral Reef Congr., 5:301-305.

Farr, M.R., 1992. Geochemical variation of dolomite cement within the Cambrian Bonneterre Formation, Missouri: evidence for fluid mixing. $J$. Sediment. Petrol., 62:636-651.

Grötsch, J., and Flügel, E., 1992. Facies of sunken Early Cretaceous atoll reefs and their capping late Albian drowning succession (northwestern Pacific). Facies, 27:153-174.

Hein, J.R., Yeh, H.-W., Gunn, S.H., Sliter, W.V., Benninger, L.M., and Wang, C.-H., 1993. Two major Cenozoic episodes of phosphogenesis recorded in equatorial Pacific seamount deposits. Paleoceanography, 8:293-311.

Hemming, G.N., Meyers, W.J., and Grams, J.C., 1989. Cathodoluminescence in diagenetic calcites: the roles of $\mathrm{Fe}$ and $\mathrm{Mn}$ as deduced from electron probe and spectrophotometric measurements. J. Sediment. Petrol., $59: 404-411$.

\footnotetext{
"Abbreviations for names of organizations and publications in ODP reference lists follow the style given in Chemical Abstracts Service Source Index (published by American Chemical Society).
} 
Hudson, J.D., 1977. Stable isotopes and limestone lithification. J. Geol. Soc. London, 133:637-660.

James, N.P., and Bone, Y., 1989. Petrogenesis of Cenozoic temperate water calcarenites, south Australia: a model for meteoric/shallow burial diagenesis of shallow water calcite sediments. J. Sediment. Petrol., 59:191-204.

Koch, R., Ogorelec, B., and Orehek, S., 1989. Microfacies and diagenesis of Lower and Middle Cretaceous carbonate rocks of NW-Yugoslavia (Slovenia, Trmovo Area). Facies, 21:135-170.

Longman, M.W., 1980. Carbonate diagenetic textures from near-surface diagenetic environments. AAPG Bull., 64:461-487.

Maliva, R.G., and Dickson, J.A.D., 1992. The mechanism of skeletal aragonite neomorphism: evidence from neomorphosed mollusks from the upper Purbeck Formation (Late Jurassic-Early Cretaceous), southern England. Sediment. Geol., 76:221-232.

McKenzie, J., Bernoulli, D., and Schlanger, S.O., 1980. Shallow-water carbonate sediments from the Emperor Seamounts: their diagenesis and paleogeographic significance. In Jackson, E.D., Koizumi, I., et al., Init. Repts. DSDP, 55: Washington (U.S. Govt. Printing Office), 415-455.

Miller, J., 1986. Facies relationships and diagenesis in Waulsortian mudmounds from the Lower Carboniferous of Ireland and N. England. In Schroeder, J.H., and Purser, B.H. (Eds.), Reef Diagenesis: Berlin (Springer), 311-335.

Moore, C.H., 1989. Carbonate Diagenesis and Porosity: Amsterdam (Elsevier), Devl. in Sedimentol. Ser., 46.

Morse, J.W., and Mackenzie, F.T., 1990. Geochemistry of Sedimentary Carbonates: Amsterdam (Elsevier), Dev. in Sedimentol. Ser., 48.

Nesse, D.G., Burr, A., Silver, A., and Bernie, B.B., 1980. Carbon isotopic signature as criterion for interpreting origin of synsedimentary cements, patch reef facies, Enewetak Atoll. AAPG Bull., 64:756.

ODP Leg 143 Shipboard Scientific Party, 1993. Examining guyots in the Mid-Pacific Mountains. Eos, 74:201-206.

Quinn, T.M., and Matthews, R.K., 1990. Post-Miocene diagenetic and eustatic history of Enewetak Atoll: model and data comparison. Geology, 18:942945.

Röhl, U., von Rad, U., and Wirsing, G., 1992. Microfacies, paleoenvironment, and facies-dependent carbonate diagenesis in Upper Triassic platform carbonates off northwest Australia. In von Rad, U., Haq, B.U., et al., Proc. ODP, Sci. Results, 122: College Station, TX (Ocean Drilling Program), 129-159.

Sager, W.W., Winterer, E.L., Firth, J.V., et al., 1993. Proc. ODP, Init. Repts., 143: College Station, TX (Ocean Drilling Program).

Saller, A.H., 1984. Petrologic and geochemical constraints on the origin of subsurface dolomite, Enewetak Atoll: an example of dolomitization by normal seawater. Geology, 12:217-220.
Saller, A.H., and Moore, C.H., Jr., 1989. Meteoric diagenesis, marine diagenesis, and microporosity in Pleistocene and Oligocene limestones, Enewetak Atoll, Marshall Islands. Sediment. Geol., 63:253-272.

Schroeder, J.H., 1988. Spatial variations in the porosity development of carbonate sediments and rocks. Facies, 18:181-204.

Strasser, A., and Davaud, E., 1986. Formation of Holocene limestone sequences by progradation, cementation and erosion: two examples from the Bahamas. J. Sediment. Petrol., 56:422-428.

Strasser, A., Strohmenger, C., Davaud, E., and Bach, A., 1992. Sequential evolution and diagenesis of Pleistocene coral reefs (South Sinai, Egypt). Sediment. Geol., 78:59-79.

Sun, S.Q., Fallick, A.E., and Williams, B.P.J., 1992. Influence of original fabric on subsequent porosity evolution: an example from the Corallian (Upper Jurassic) reefal limestones, the Weald Basin, southern England. Sediment. Geol., 79:139-160.

Thein, J., 1985. Die Paläogeographie des Turon im Hohen Atlas Marokkos: Ergebnisse geochemischer und mikrofazieller Untersuchungen. Habilitation thesis.

Tucker, M.E., 1990. Burial diagenesis. In Tucker, M.E., and Wright, V.P. (Eds.), Carbonate Sedimentology: Oxford (Blackwell).

van Waasbergen, R.J., and Winterer, E.L., 1993. Summit geomorphology of Western Pacific guyots. In Pringle, M.S., Sager, W.W., Sliter, W.V., and Stein, S. (Eds.), The Mesozoic Pacific: Geology, Tectonics, and Volcanism. Geophys. Monogr., Am. Geophys. Union, 77:335-366.

Vollbrecht, R., and Meischner, D., 1993. Sea level and diagenesis: a case study on Pleistocene beaches, Whalebone Bay, Bermuda. Geol. Rundsch., $82: 248-262$

Walkden, G.M., and Berry, J.R., 1984. Syntaxial overgrowth in muddy crinoidal limestones: cathodoluminescence sheds new light on an old problem. Sedimentology, 31:251-267.

Winterer, E.L., 1991. The Tethyan Pacific during Late Jurassic and Cretaceous times. Palaeogeogr., Palaeoclimatol., Palaeoecol., 87:253-265.

Wright, V.P., 1992. A revised classification of limestones. Sediment. Geol., $76: 177-185$

Zempolich, W.G., 1989. Meteoric stabilization and preservation of limestone within the Late Proterozoic Beck Spring Dolomite of eastem California. In Cooper, J.D., Albright, G., Griffin, K.M., McCutcheon, K.F., and Zempolich, W.G. (Eds.), Calvacade of Carbonates. SEPM Field Trip Guidebook, 61:61-75.

Date of initial receipt: 15 November 1993

Date of acceptance: 6 July 1994

Ms 143SR-224 

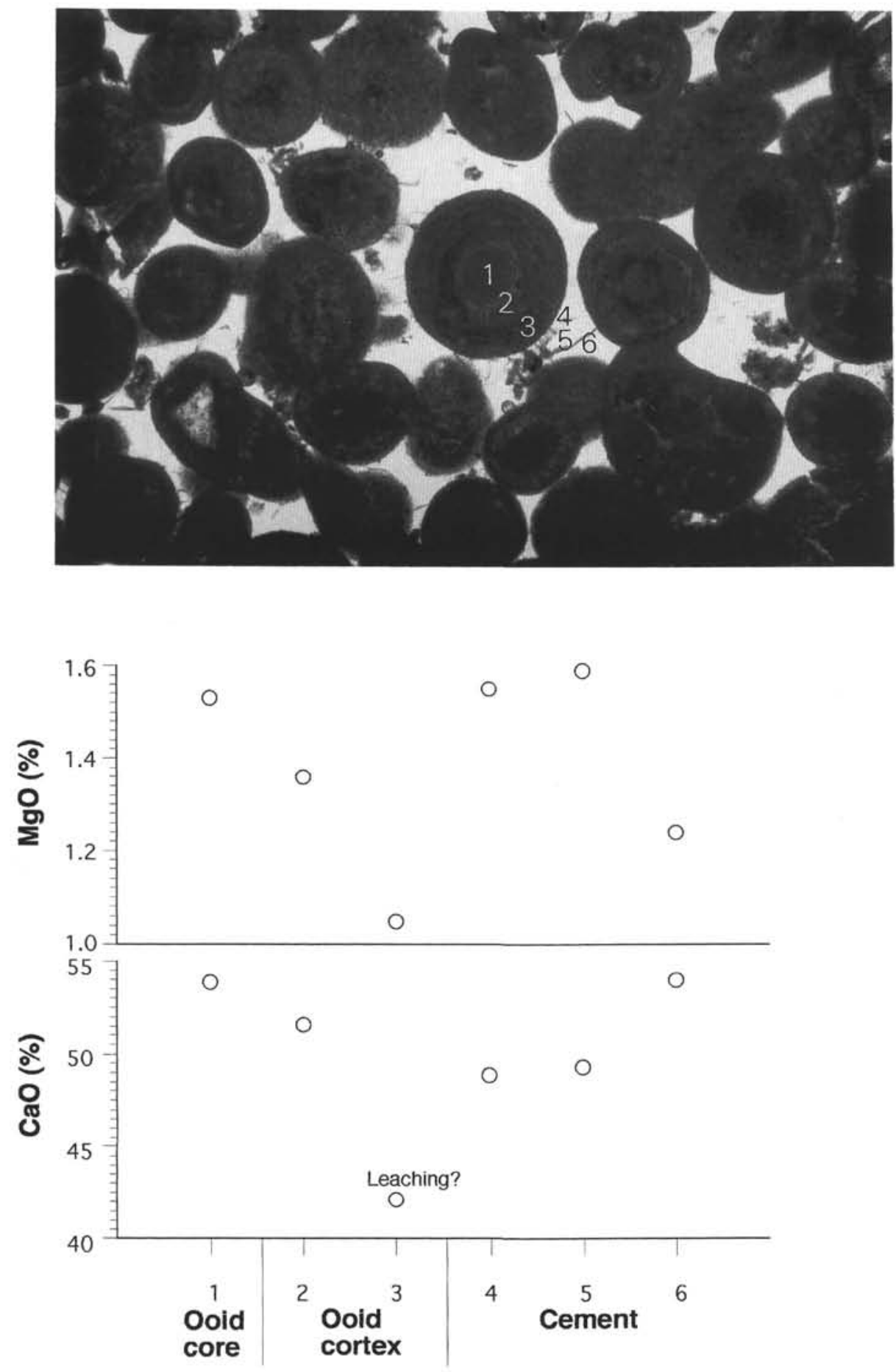

Figure 7. $\mathrm{CaO}$ and $\mathrm{MgO}$ microprobe analyses of Sample $143-866 \mathrm{~A}-129 \mathrm{R}-1,124-128 \mathrm{~cm}$. 

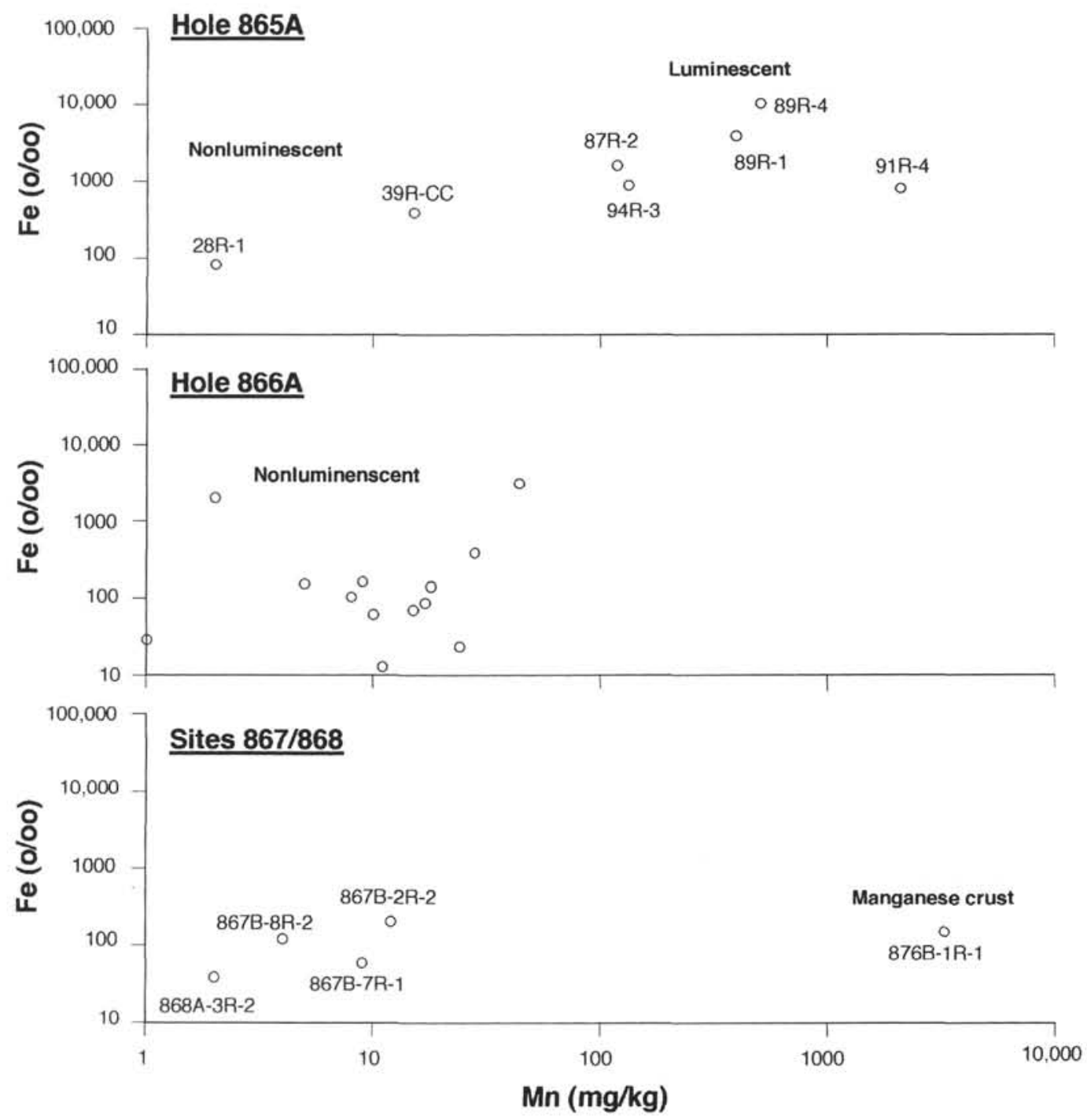

Figure 8. Mn vs. Fe contents of cements (ICP-AES, AA analyses). For further explanation see text. 


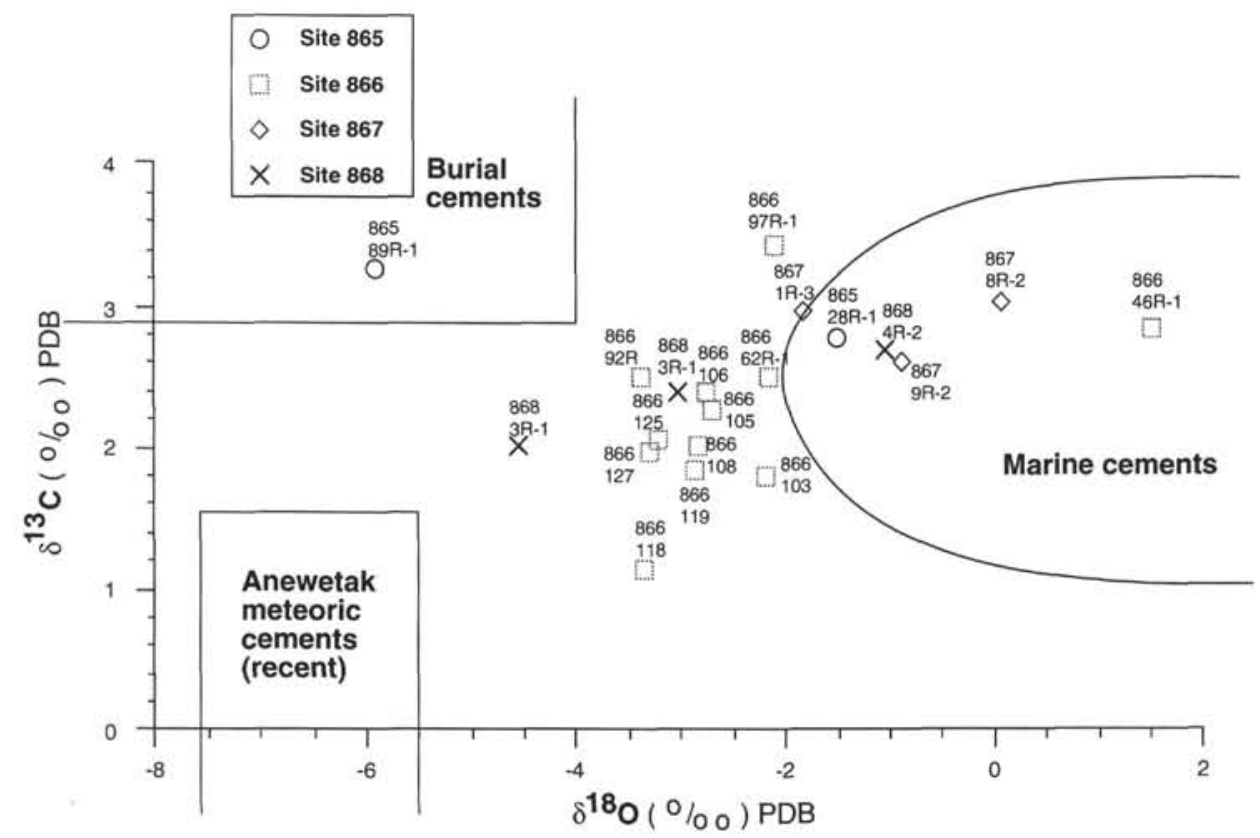

Figure 9. Stable isotope analyses of selected cement samples (Sites 865 to 868). For further explanation see text. 
Sites $865,866,867$, and 868
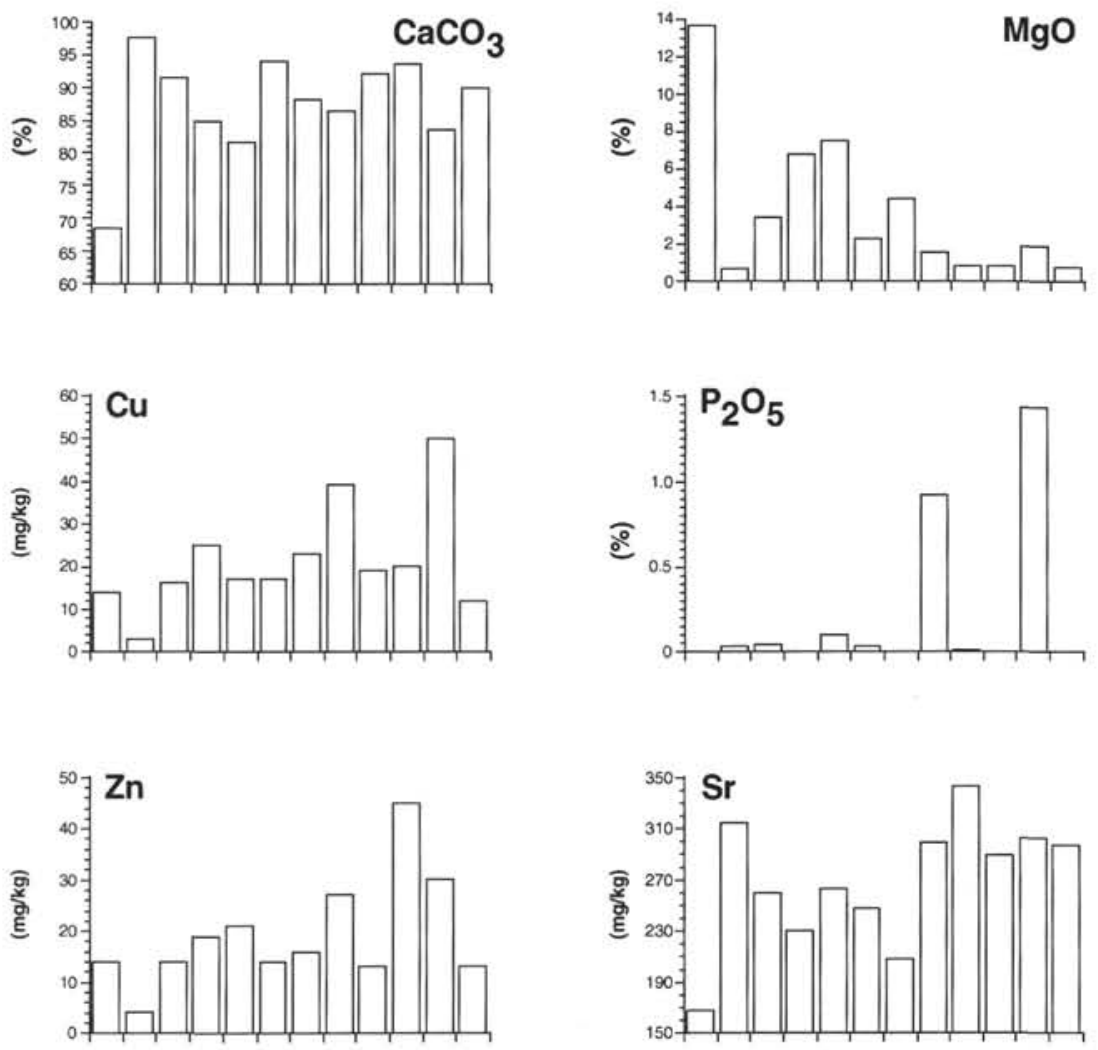

Figure 10. A. $\mathrm{CaCO}_{3}, \mathrm{MgO}, \mathrm{Cu}, \mathrm{P}_{2} \mathrm{O}_{5}, \mathrm{Zn}, \mathrm{Sr}, \mathrm{SO}_{3}$, and Ba contents. B. $\mathrm{Zr}, \mathrm{V}, \mathrm{MnO}, \mathrm{SiO}_{2}, \mathrm{Fe}_{2} \mathrm{O}_{3}$, and $\mathrm{Al}_{2} \mathrm{O}_{3}$ contents vs. different microfacies types.
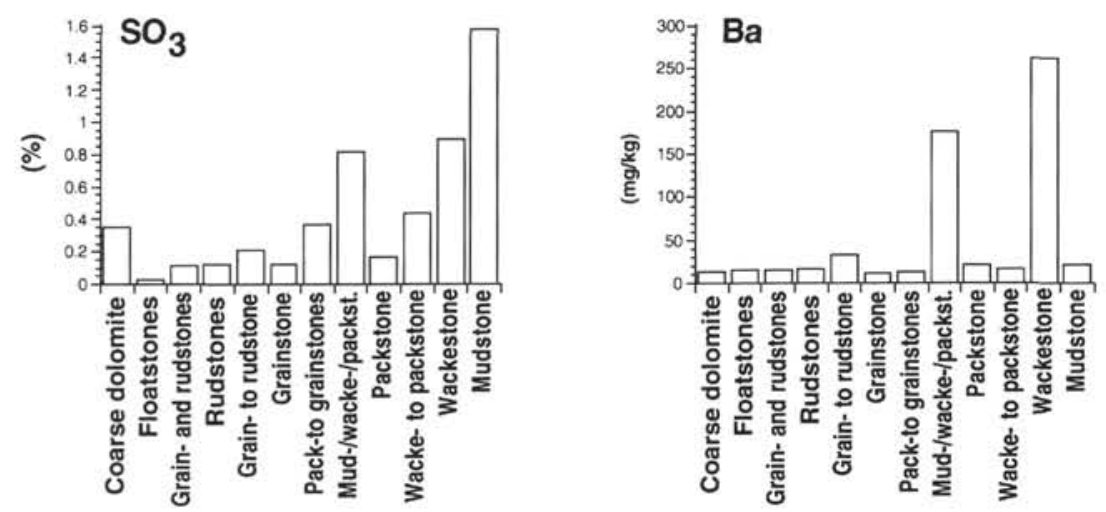
Sites $865,866,867$, and 868
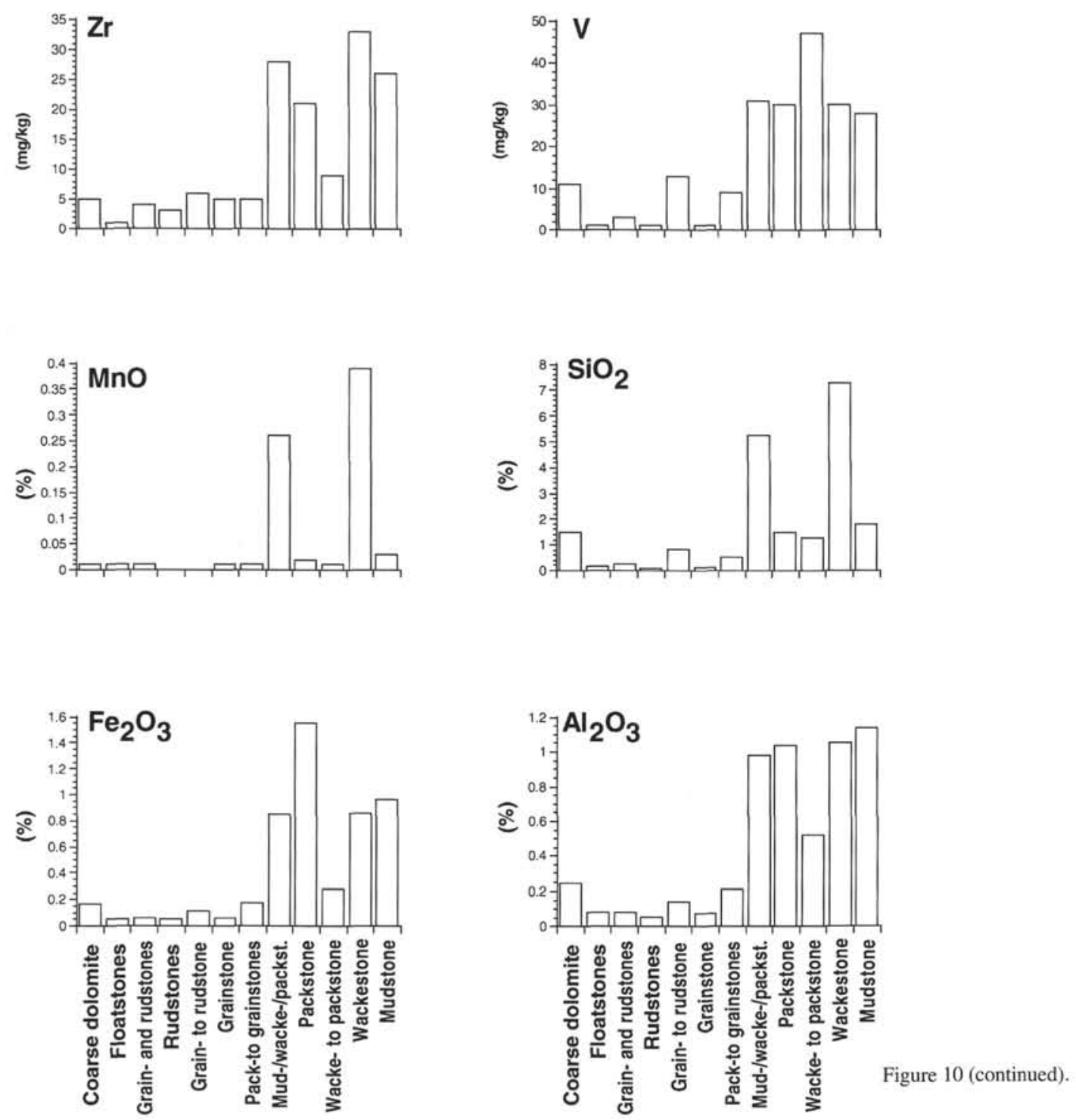
U. RÖHL, A. STRASSER

Figure 11. Cluster analysis of both microfacies and chemistry data. For further explanation see text.

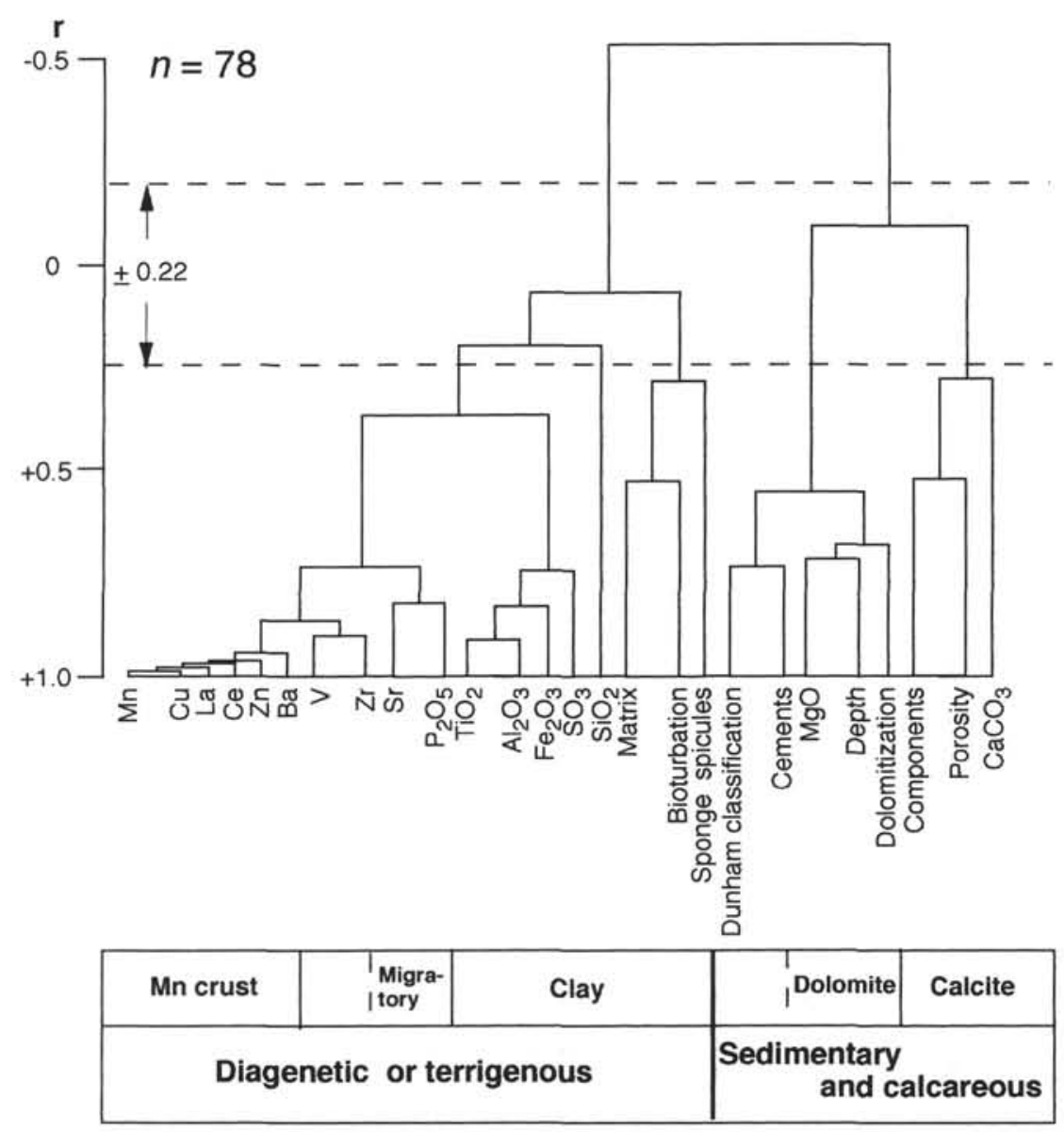



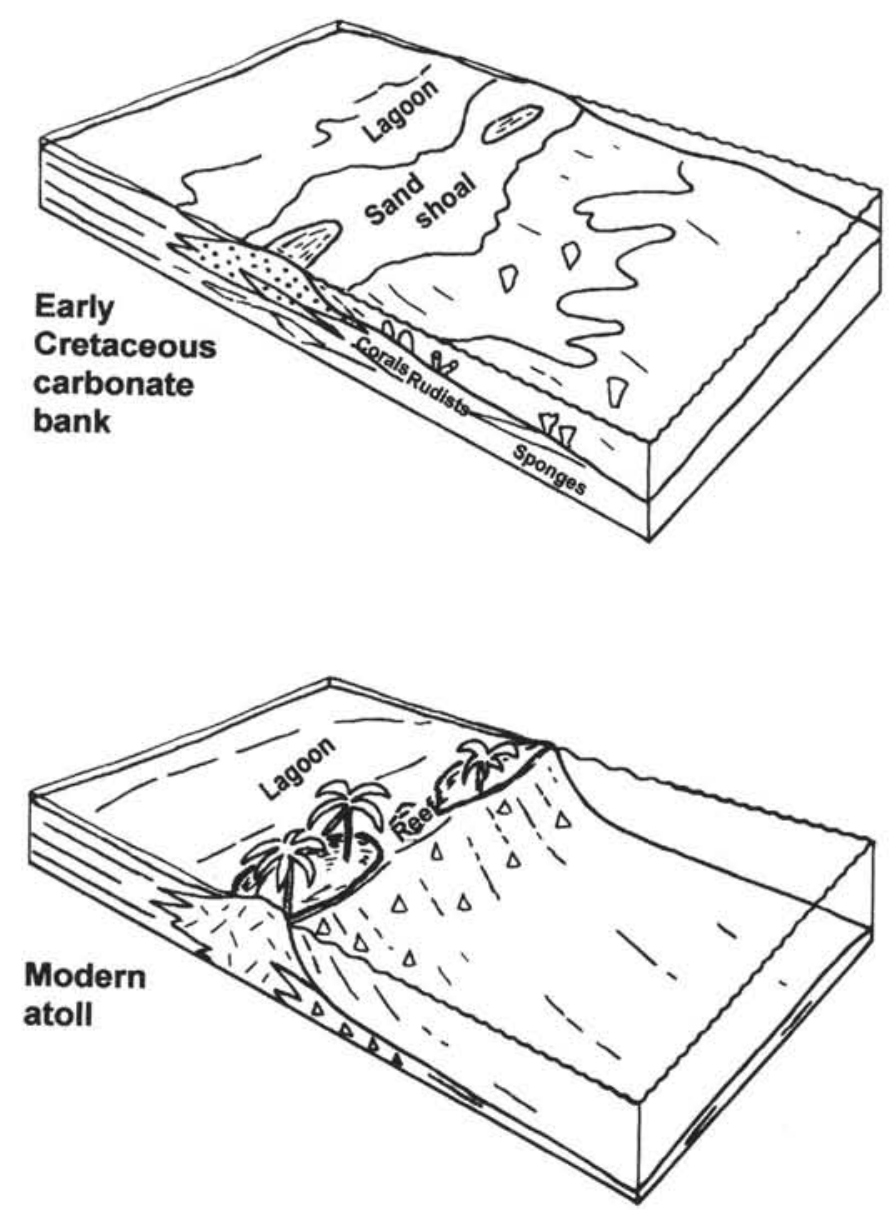

Figure 12. Morphology and facies differences between modern atolls and Lower Cretaceous guyots. 


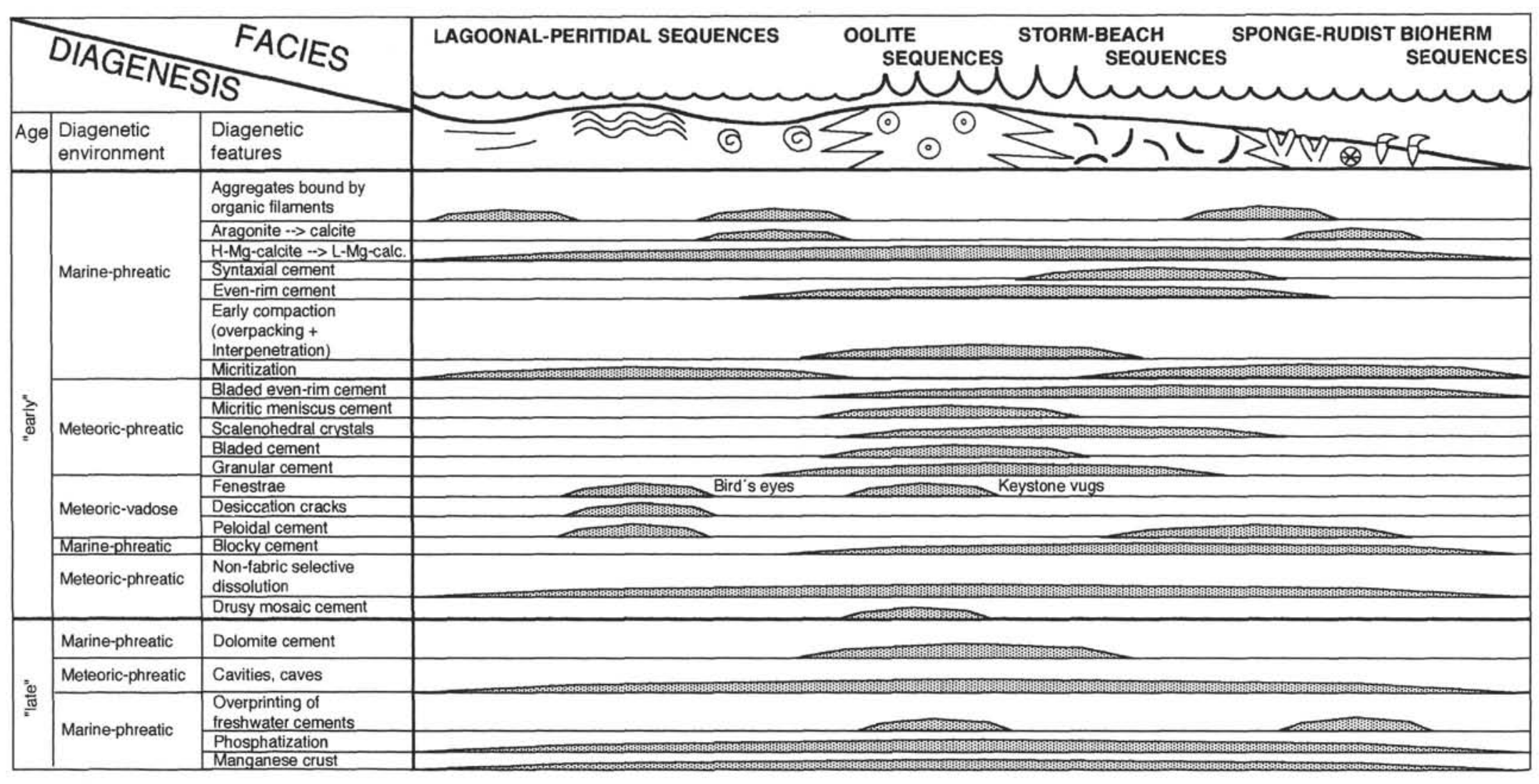

Figure 13. Diagenetic model. For further explanation see text. 


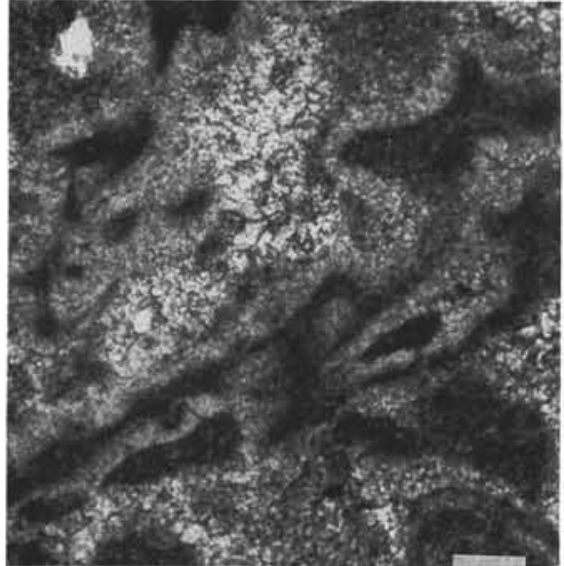

1

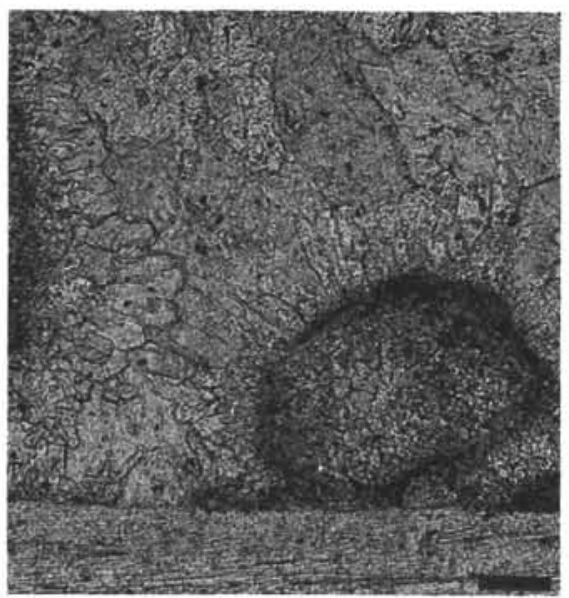

4

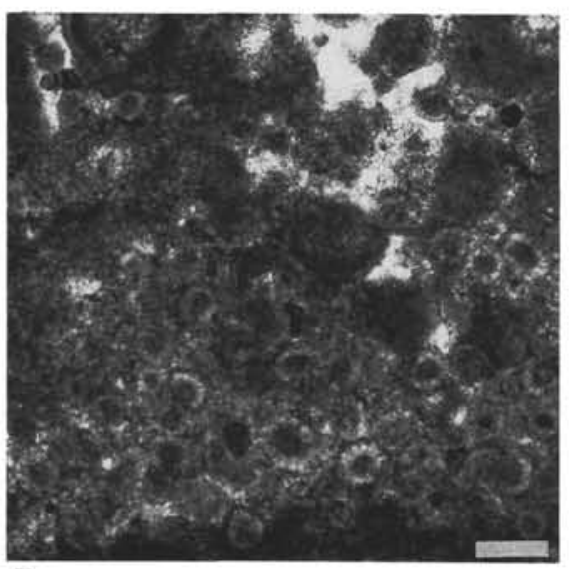

7

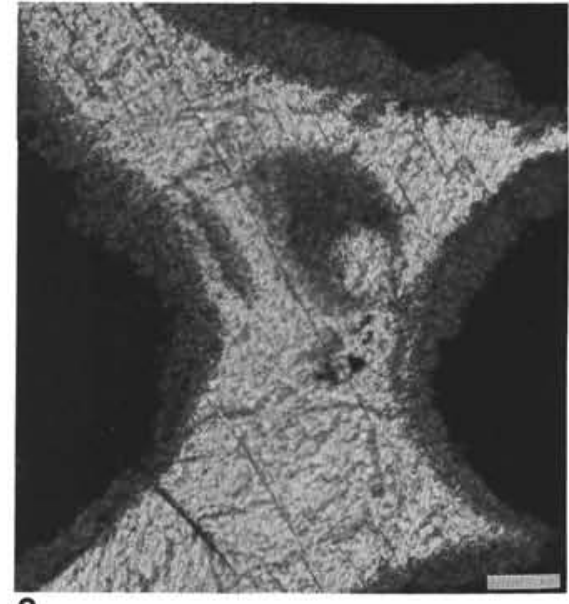

2
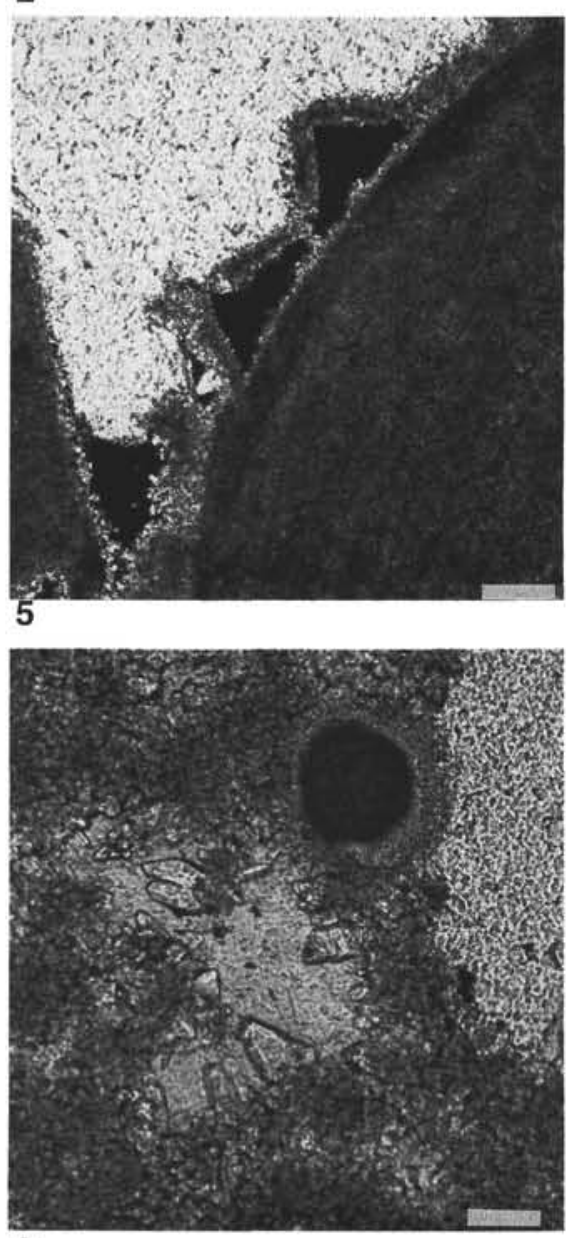

8

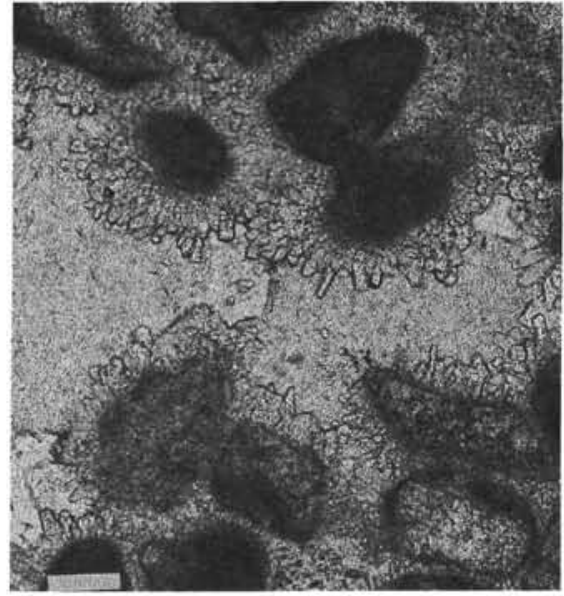

3

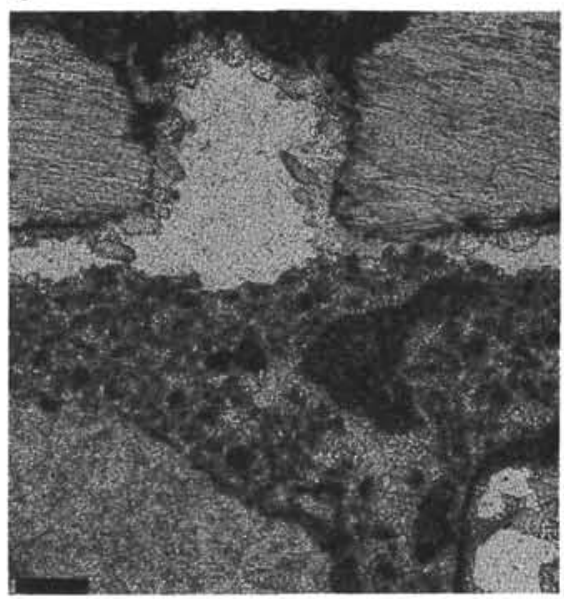

6

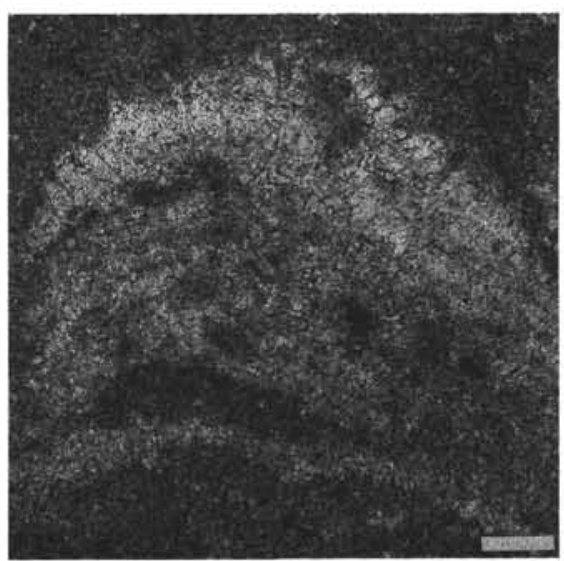

9

Plate 1. 1. Recrystallized micritic matrix and fragment of calcareous algae; pore filled by medium-grained cement probably of fresh-water origin, Sample 143-865A-34R-1, 8-9 cm, scale $0.1 \mathrm{~mm}$. 2. Micritic, probably marine-phreatic cement (even-rim cement) coating ooids. Pore space filled with blocky meteoric-phreatic cement, Sample 143-866A-164R-1, 140-143 cm, scale $0.1 \mathrm{~mm}$. 3. Thick coating of thinly bladed marine-phreatic cement with some scalenohedral crystal terminations. Pore space is almost completely filled by blocky meteoric-phreatic cement, Sample 143-868A-1R-2, 15-17 cm, scale 0.1 $\mathrm{mm}$. 4. Bladed crystals and clearly visible scalenohedral terminations suggest an original composition of high-Mg calcite formed in a marine-phreatic diagenetic environment. Pores filled with blocky meteoric-phreatic cement, Sample 143-867B-1R-3, 15-18 cm, scale 0.05 mm. 5. Early compaction caused spalling of the outermost ooid layer and early fringing cement. Pore space partly filled with coarse meteoric-phreatic cement, black = porosity, Sample 143-866A-157R-2, $84-86 \mathrm{~cm}$, scale $0.05 \mathrm{~cm}$. 6. Peloidal cement of possibly high-Mg calcite origin behaved like an internal sediment and partly filled pore space in a marine-phreatic environment, Sample 143-867B-8R-2, 30-33 cm, scale 0.2 mm. 7. Spherulites of probable microbial origin in an algal mat, Sample 143-866A-71R-1, 64-67 $\mathrm{cm}$, scale $0.1 \mathrm{~mm}$. 8. Spherulite and early cement growing in pore space, Sample 143-866A-148R-1, 4-7 cm, scale $0.05 \mathrm{~mm}$. 9. Caliche crust formed in a freshwater diagenetic environment which periodically underwent subaerial exposure, Sample 143-866A-39R-1, 23-24 cm, scale $0.1 \mathrm{~mm}$. 


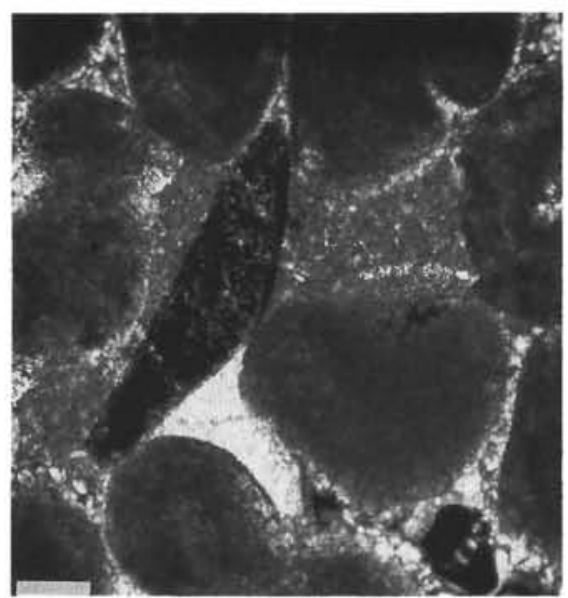

1

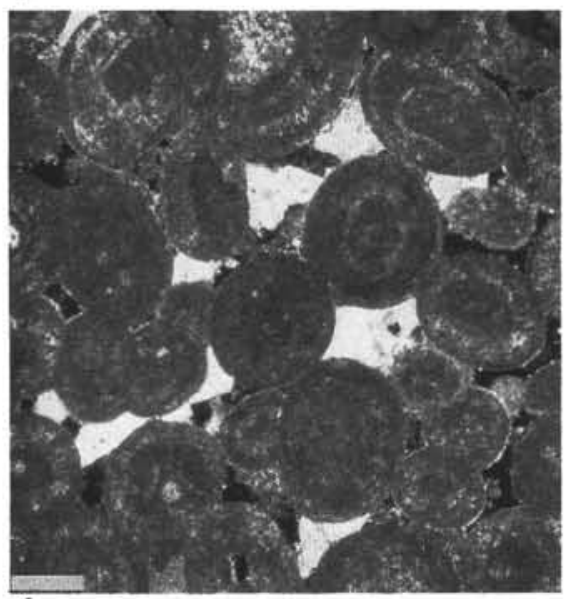

4

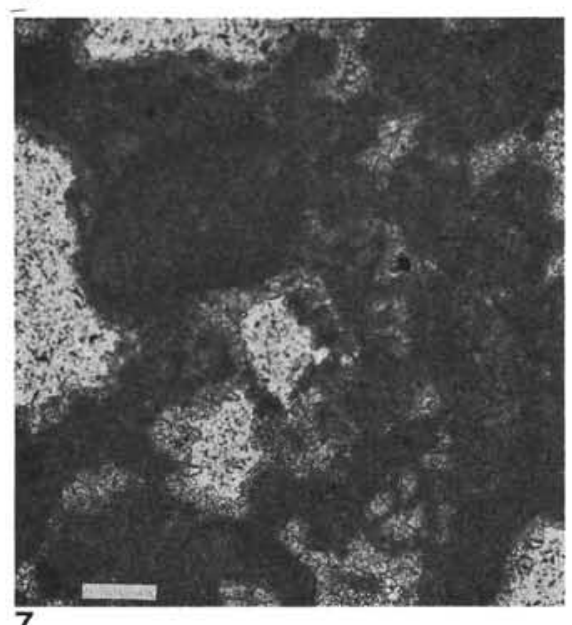

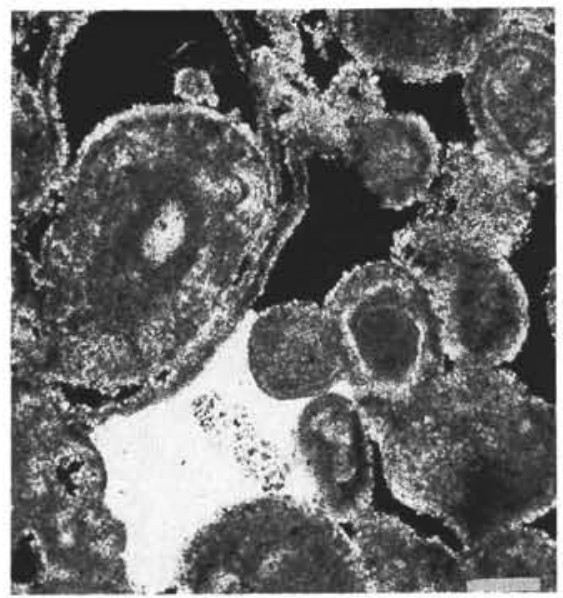

2

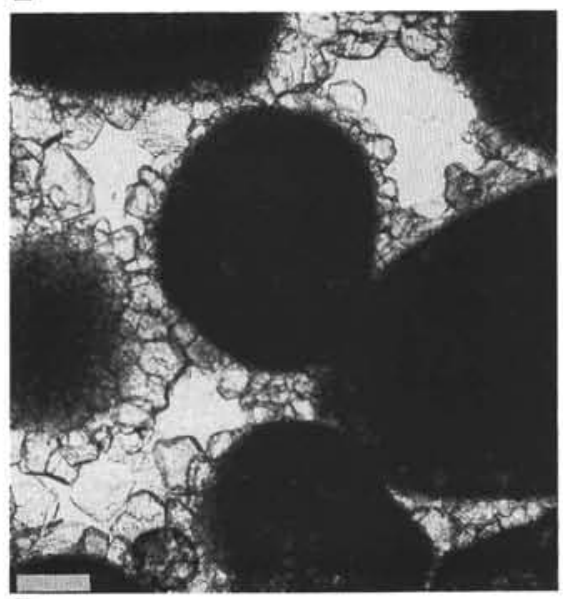

5

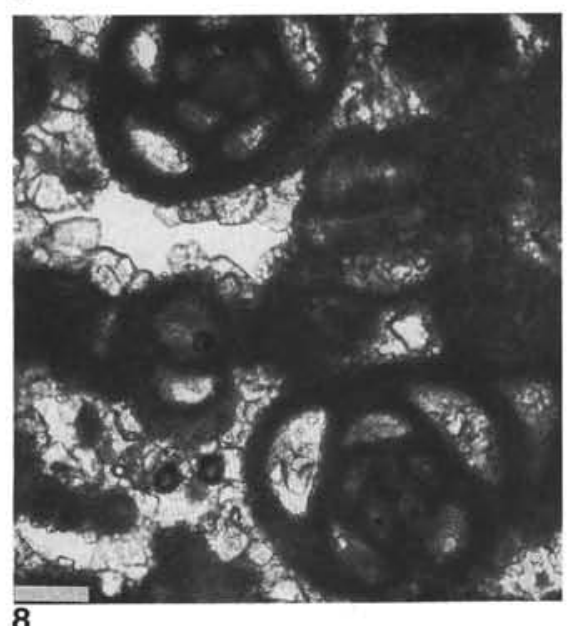

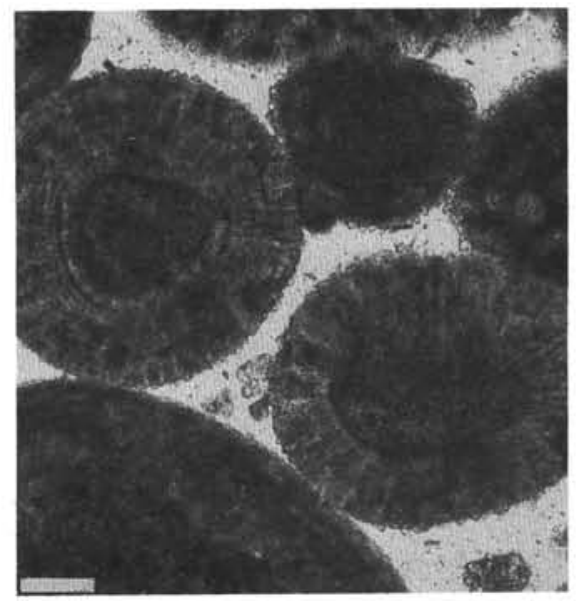

3

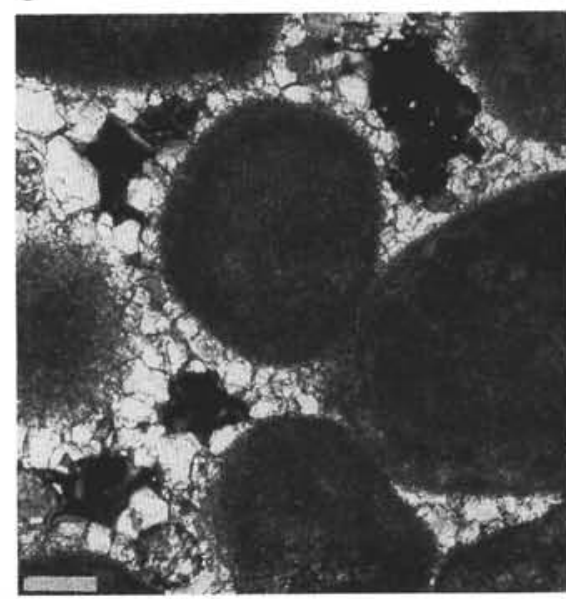

6

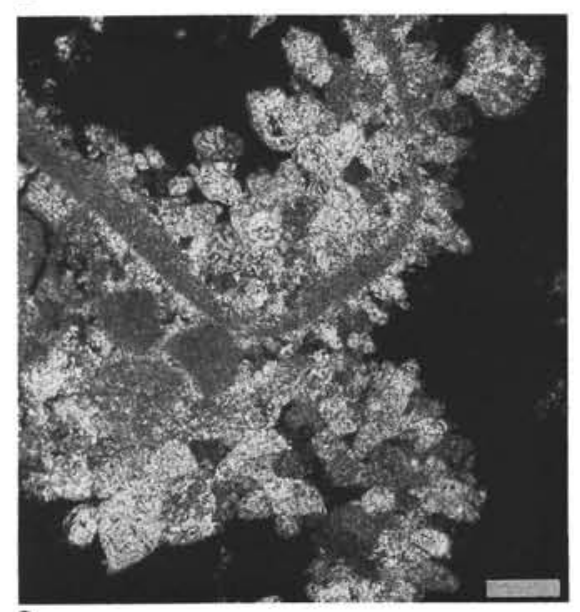

9

Plate 2. 1. Syntaxial cement surrounding echinoderm fragment, oolitic grainstone, Sample 143-866A-77R-3,90-94 cm, crossed nicols, scale $0.1 \mathrm{~mm}$. 2. Early precipitation of syntaxial cement around echinoderm fragment prevented compaction of this grainstone. Note collapsed large ooid, suggesting that part of the cortex (aragonite?) was dissolved during freshwater diagenesis, black = porosity, Sample 143-866A-78R-3, 90-91 cm scale 0.1 mm. 3. Barely cemented oolite, and ooids displaying radial cortices, Sample 143-866A-78R-2, $85-87 \mathrm{~cm}$, scale $0.1 \mathrm{~mm}$. 4. Blocky calcite cement (light) of meteoric-phreatic origin. Note overpacking resulting from compaction prior to freshwater cementation in the oolitic grainstone, Sample 143-866A-129R-1, 124-128 cm, crossed nicols, scale $0.3 \mathrm{~mm}$. 5. Coarse meteoric-phreatic cement in oolitic grainstone, Sample 143-866A-74R-2, 94-97 cm, scale 0.1 mm. 6. As Figure 5, but crossed nicols. 7 . Micritic meniscus cement indicating a vadose diagenetic environment. Glaebules point to pedogenetic overprinting of originally lagoonal facies, Sample 143-866A-25R-1, 68-70 cm, scale $0.1 \mathrm{~mm}$. 8. Intraparticle and interparticle porosity partly filled by coarse freshwater cement, bioclastic packstone to grainstone, Sample 143-866A-76R-1, 62-64 cm, scale $0.1 \mathrm{~mm}$. 9. Intense carbonate dissolution leading to high interparticle and intraparticle porosity resulted from percolating freshwater. Only the micritic envelope of the original particles is preserved. Cements are phreatic and probably of freshwater origin; Sample 143-866A-94R-1, 8-9 cm, crossed nicols, scale $0.1 \mathrm{~mm}$. 


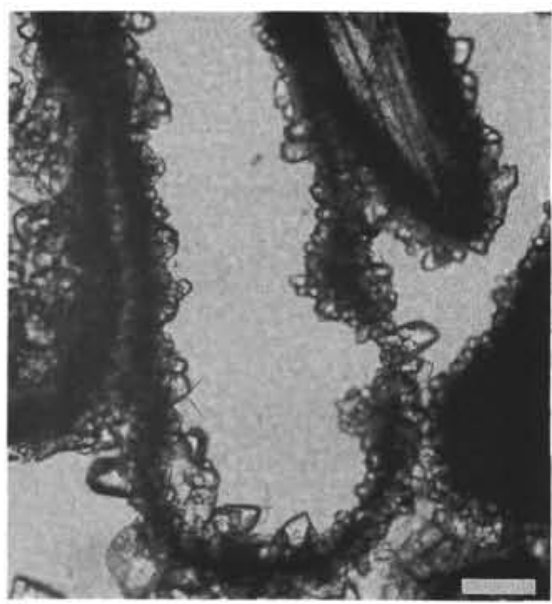

1

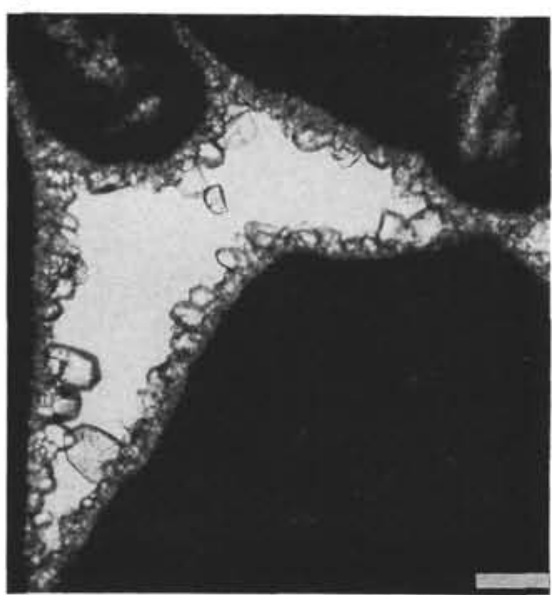

4

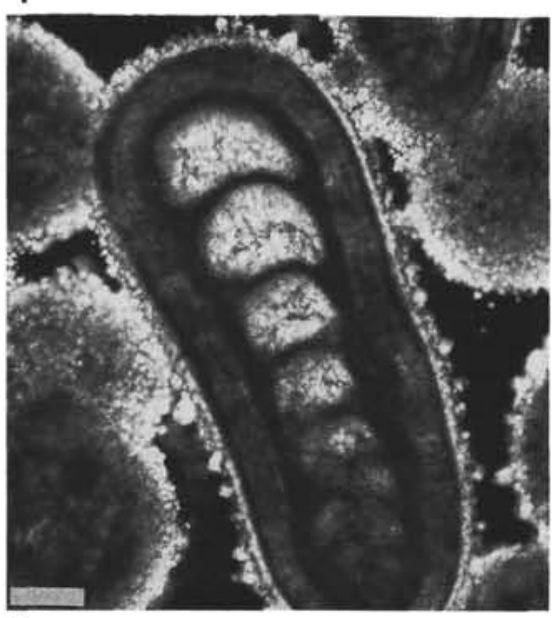

7

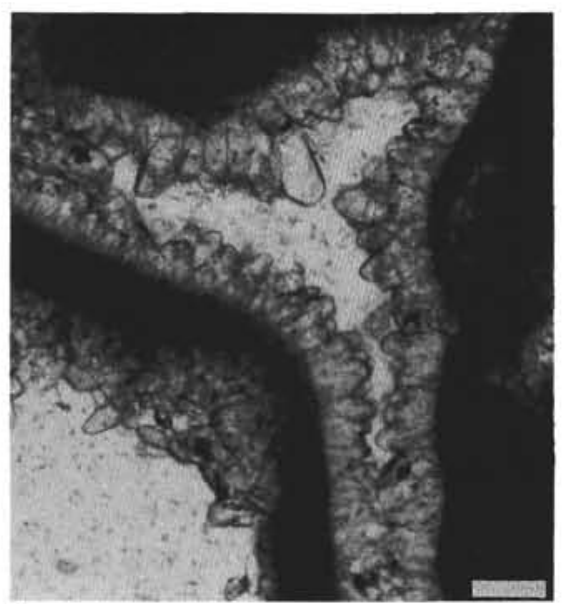

2

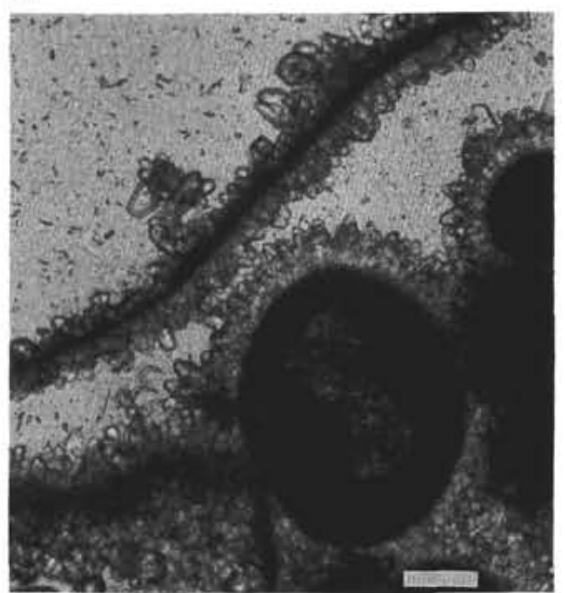

5

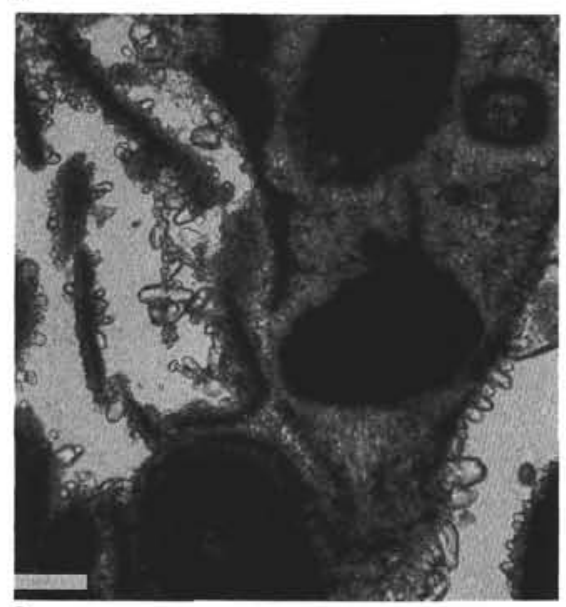

8

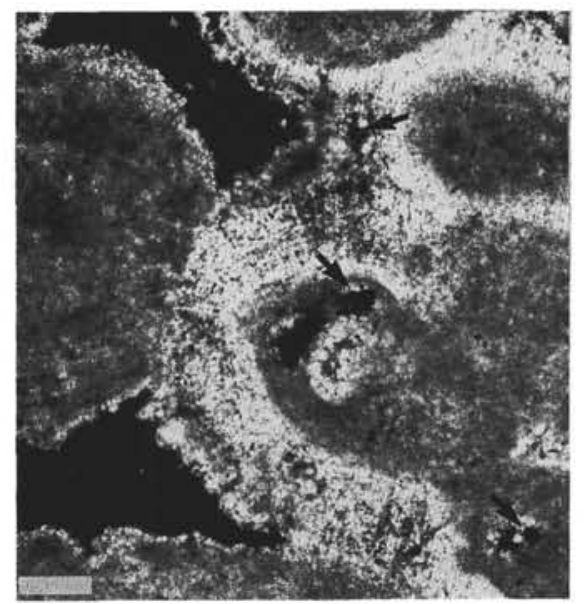

3

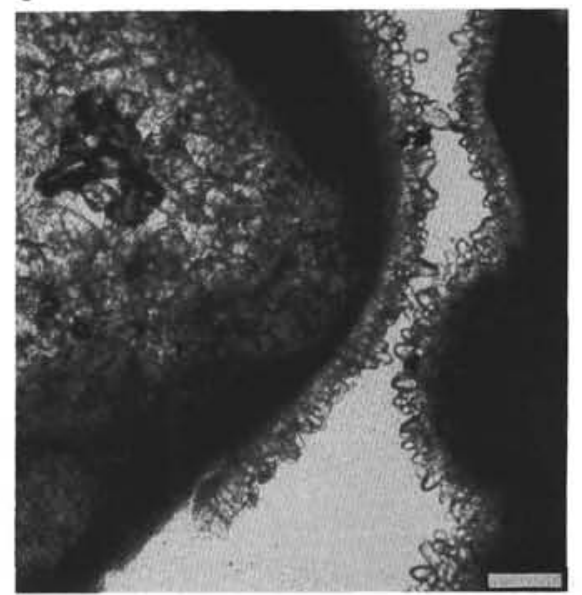

6

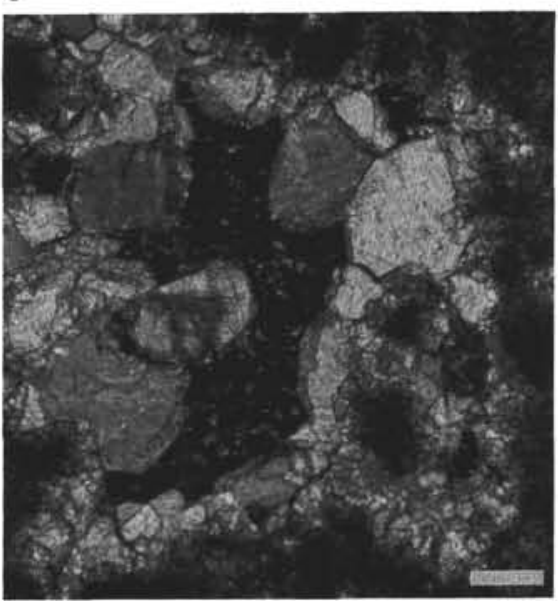

9

Plate 3. 1. Scalenohedral crystal terminations on micritized, dissolution-resistant rim of pelecypod fragment, Sample $143-867 \mathrm{~B}-4 \mathrm{R}-1,68-70 \mathrm{~cm}$, scale $0.1 \mathrm{~mm}$. 2. Bladed even-rim cement with scalenohedral terminations, Sample 143-866A-94R-1, 31-33 cm, scale $0.1 \mathrm{~mm}$. 3. Reworked hardground piece with thick fibrous cement of marine-phreatic origin, microbially induced framboidal pyrite (arrows), and interparticle porosity, Sample 143-866A-78R-3, 90-91 cm, scale $0.1 \mathrm{~mm}$. 4. Scalenohedral crystal terminations, Sample 143-867B-4R-1, 68-70 cm, scale $0.1 \mathrm{~mm}$. 5. Preserved ooids, dissolved pelecypod shells except for outer micritized part, fringing cements, and scalenohedral crystals, Sample 143-866A-93R-1, 17-19 cm, scale 0.1 mm. 6. Recrystallized and micritized shell fragment, fringing cements, and scalenohedral crystal terminations, Sample 143-866A-95R-1, 21-24 cm, scale $0.1 \mathrm{~mm}$. 7. Foraminifer as ooid core, cemented single chambers of test, early fringing cement, and scalenohedral terminations, Sample 143-867B-6R-1, 35-37 cm, crossed nicols, scale 0.1 mm. 8. Oolitic grainstone, ooids are partly well cemented with thick fringing cement layers, micritized parts of shells are not dissolved, and scalenohedral terminations, Sample 143-866A-75R-3, 67-69 cm, scale $0.1 \mathrm{~mm}$. 9. Blocky calcite cement in dissolution void of peloidal packstone, Sample 143-866A-47R-1, 38-40 cm, crossed nicols, scale $0.1 \mathrm{~mm}$. 


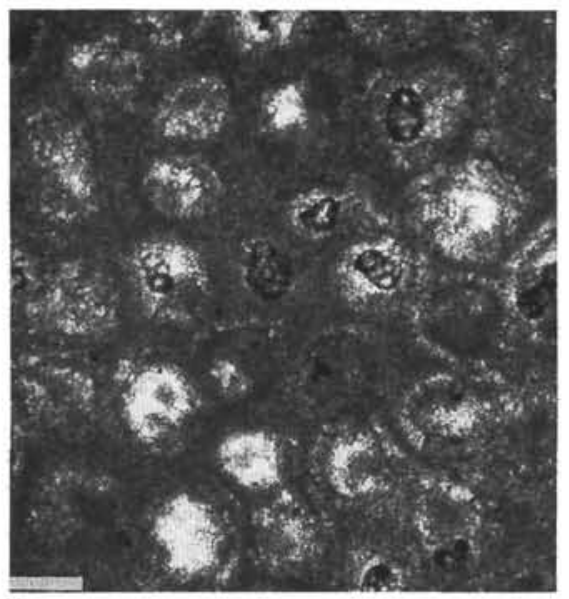

1

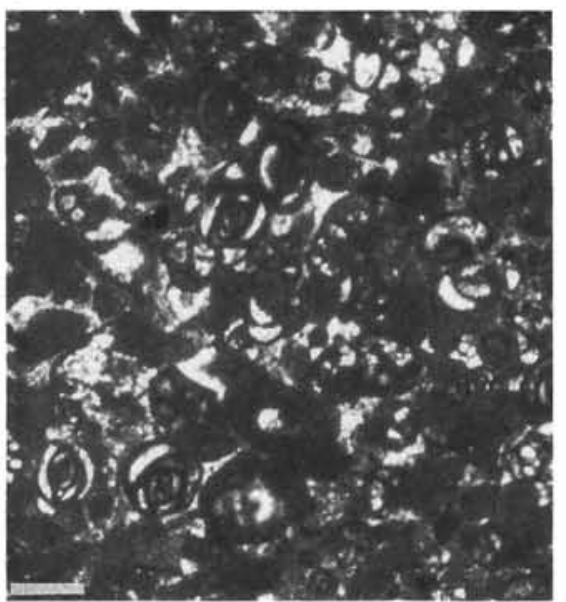

4

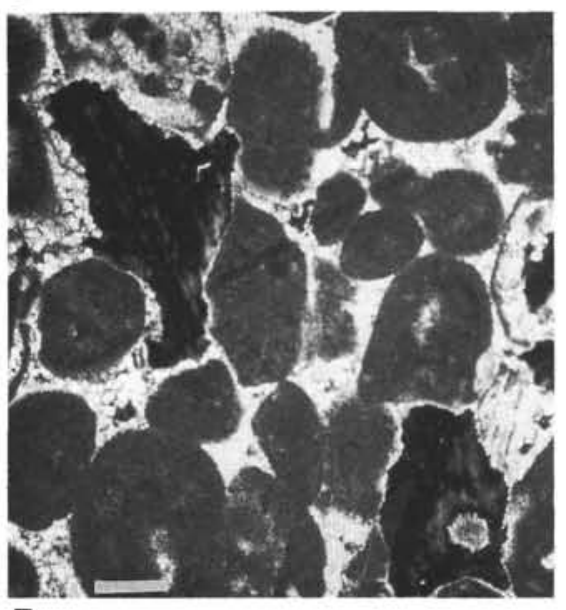

7

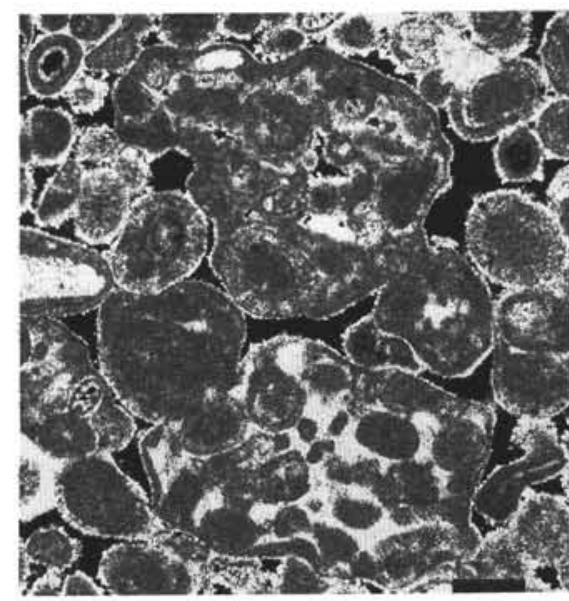

2

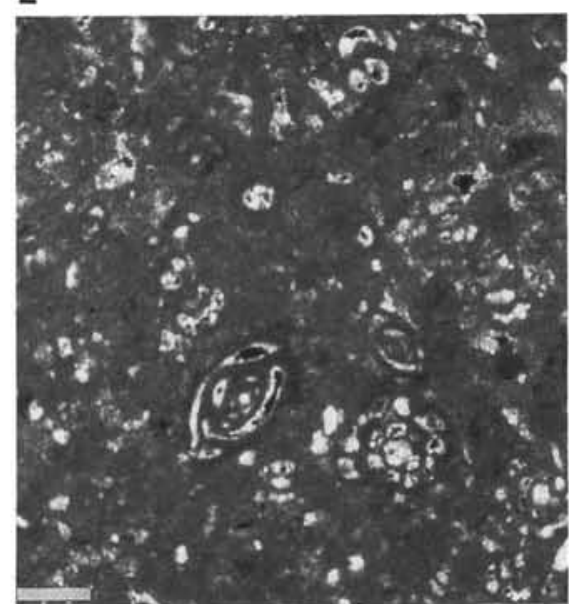

5

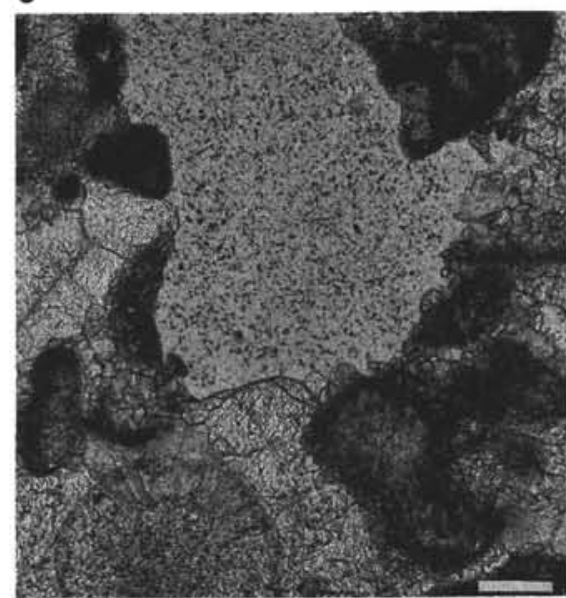

8

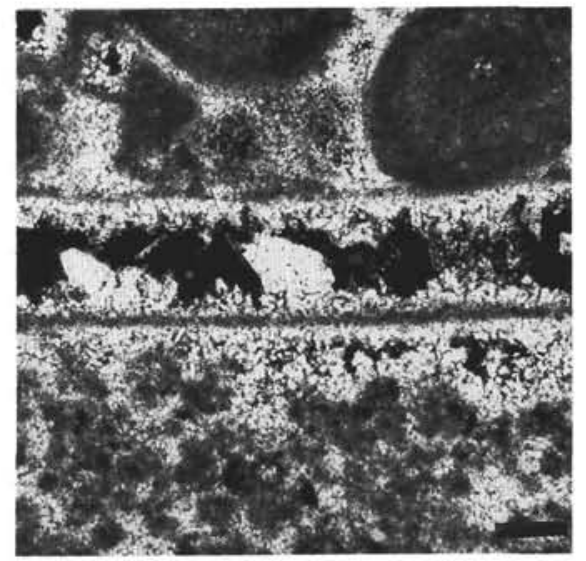

3

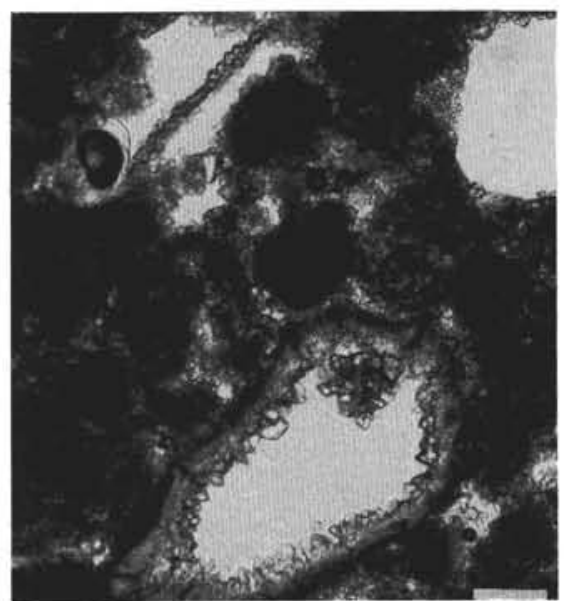

6

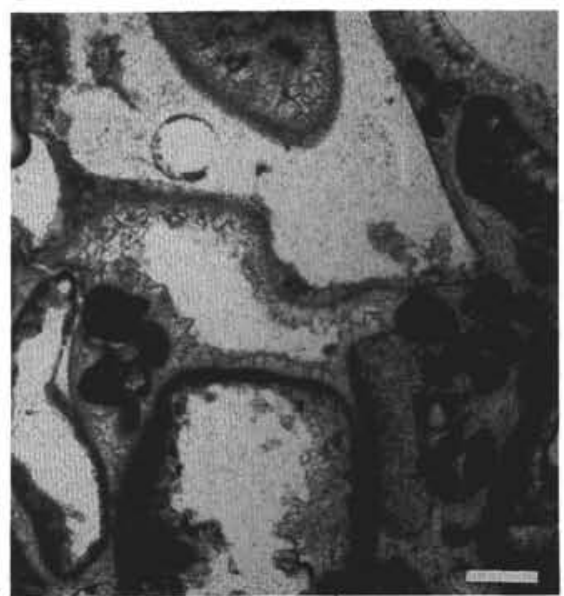

9

Plate 4. 1. Intraparticle porosity, calcareous algae, Sample 143-866A-55R-CC, 30-31 cm, scale $0.1 \mathrm{~mm}$. 2. Interparticle porosity (black), grapestones, Sample 143-866A-153R-2, 20-22 cm, crossed nicols, scale $0.2 \mathrm{~mm}$. 3. Peloidal cement, moldic porosity within shell fragment, only micritized part of shell is retained, pore space partly reduced by growing of early fringing cement and some blocky calcite crystals, Sample 143-866A-95R-1, 26-28 cm, scale 0.1 mm. 4 . Interparticle and intraparticle porosity of foraminiferal (miliolids) packstone, Sample $143-866 \mathrm{~A}-70 \mathrm{R}-1,1-3 \mathrm{~cm}$, scale $0.3 \mathrm{~mm}$. 5. Porosity of foraminiferal wackestone, Sample 143-866A-70R-1, 1-3cm, scale 0.3 mm. 6. Moldic porosity, even-rim cements with scalenohedral terminations, Sample 143-866A-58R-1, 72-75 cm, scale $0.1 \mathrm{~mm}$. 7. Dissolution on grain surfaces by fresh water in oolitic grainstone, Sample 143-866A-74R-2, 94-97 cm, scale 0.3 mm. 8. Enlargement of keystone vug by destructive, nonselective dissolution within peloidal grainstone, Sample 143-865A-34R-1, 15-16 cm, scale 0.1 mm. 9. Interparticle and intraparticle porosities in bioclastic grainstone to rudstone, note relatively thick crust of fringing cement, partly with scalenohedral crystal terminations, Sample 143-866A-94R-1, 31-33 cm, scale $0.3 \mathrm{~mm}$. 


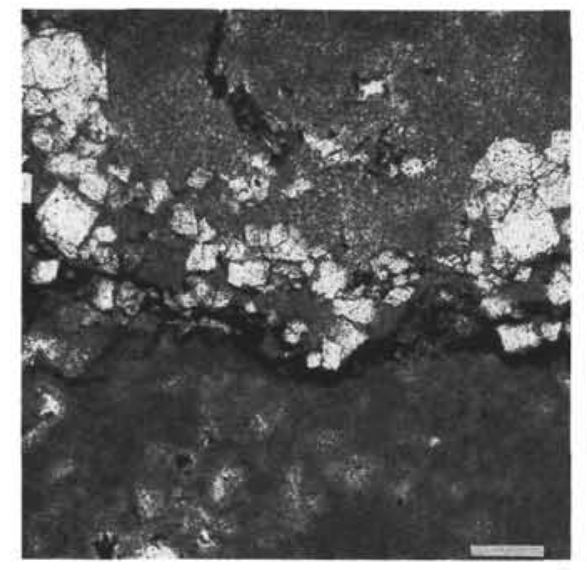

1
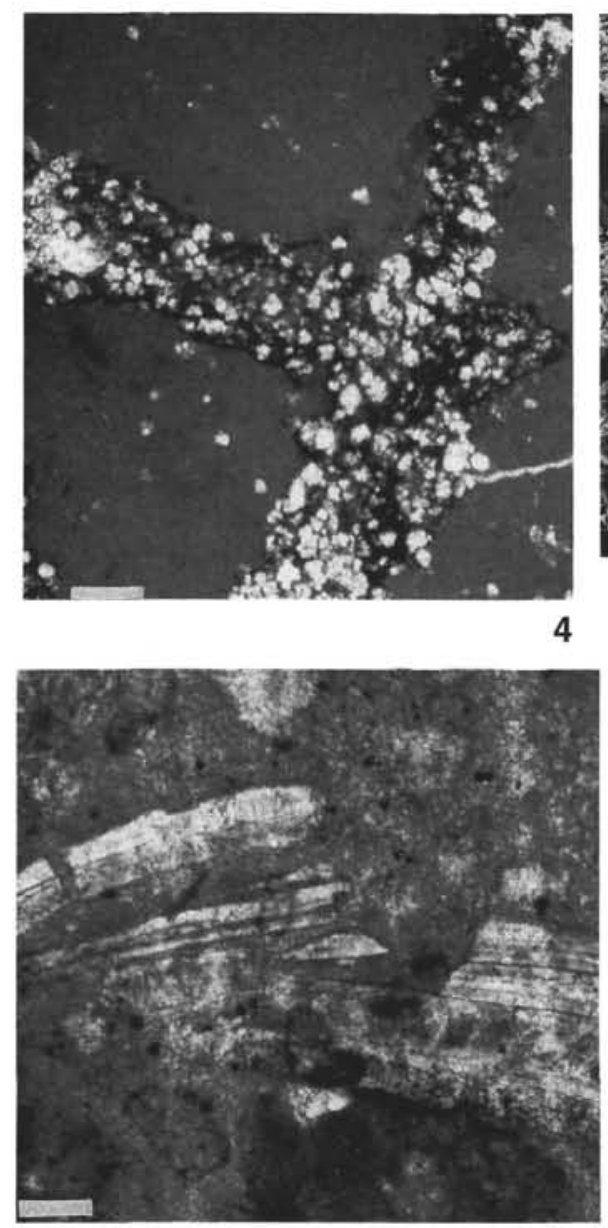

7

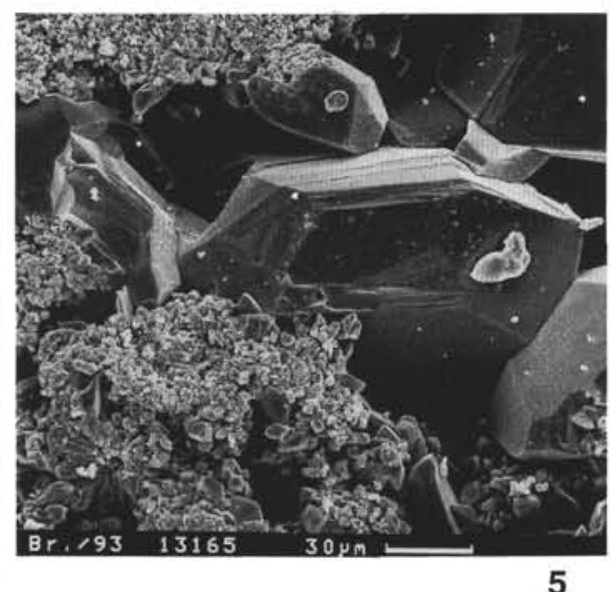

5

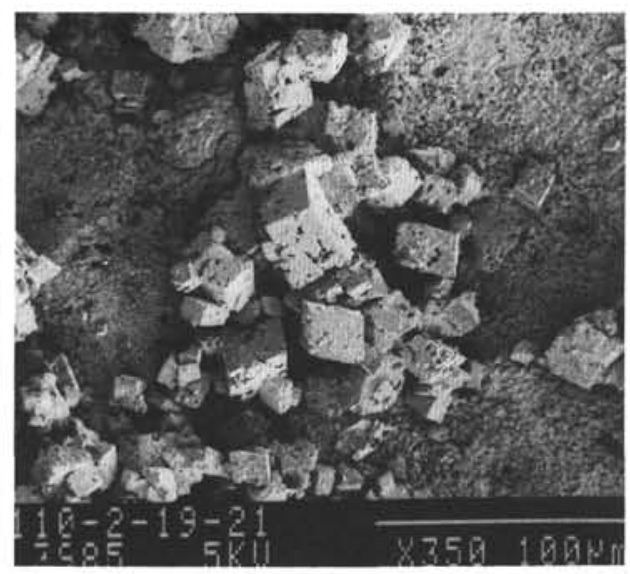

2
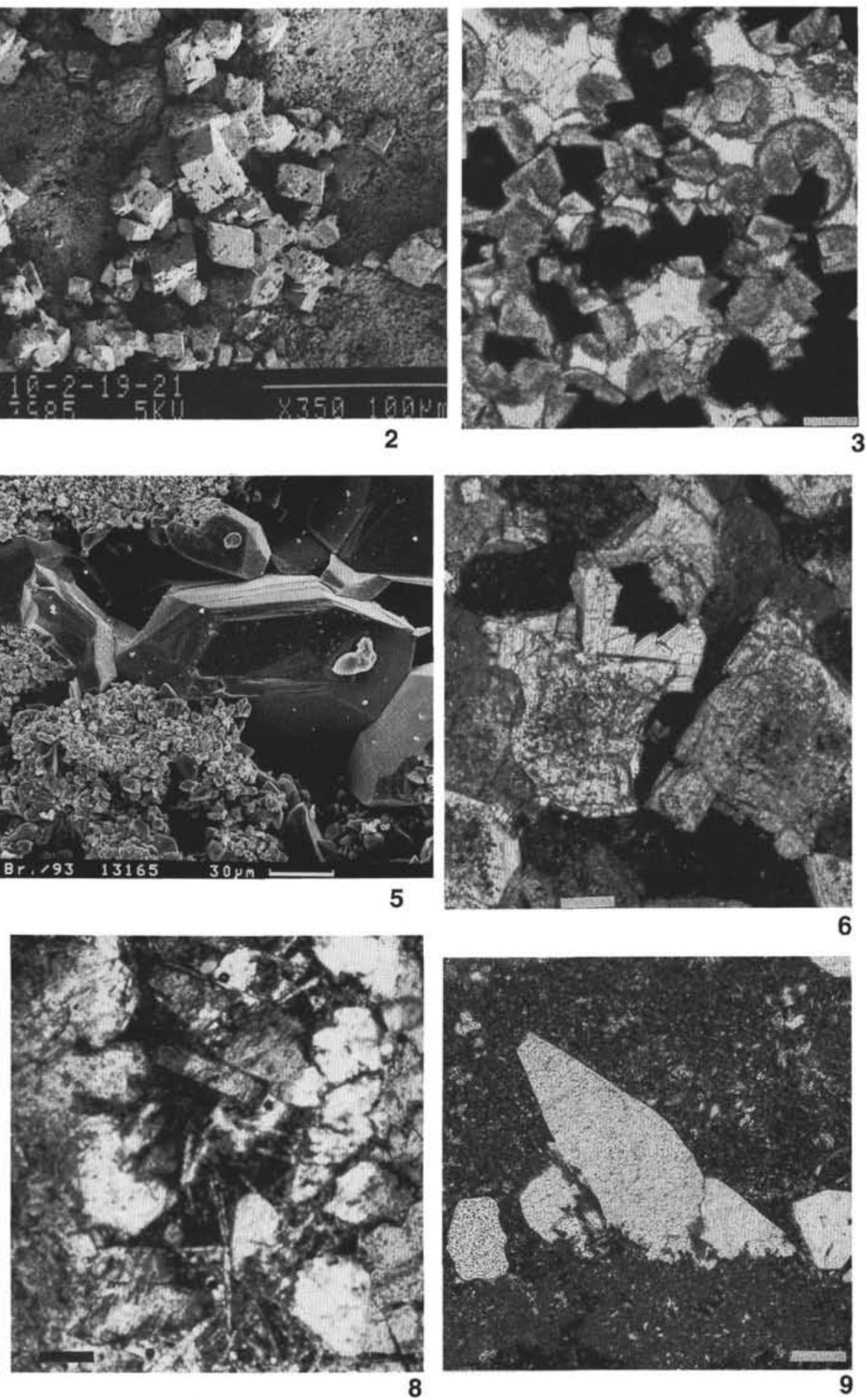

Plate 5. 1. Early dolomitization, clay layers sealed underlying strata against dolomitizing fluids, Sample $143-866 \mathrm{~A}-119 \mathrm{R}-1,70-72 \mathrm{~cm}, \mathrm{scale} 0.1 \mathrm{~mm} . \quad 2$. Early dolomitization, single dolomite rhombs exhibit dissolution features, Sample 143-866A-110R-2, 19-21 cm, SEM, slightly etched with acid, scale 0.1 mm. 3. Strongly dolomitized oolitic grainstone, black = porosity, the ooids appear as ghosts formed by inclusion-rich dolomite. The early fringing cements retained a calcitic mineralogy (stained by Alizarin-Red-S), Sample 143-866A-168R-1, 108-109 cm, scale $0.3 \mathrm{~mm}$. 4. Early dolomitization started in millimeter-scale cracks, Sample 143-866A-138R-1, 22-24 cm, scale $0.3 \mathrm{~mm}$. 5. Dolomitic blocky cement, Sample 143-866A-168R-3, 78-79 cm, scale 0.03 mm. 6. Intracrystalline and intercrystalline porosity in dolomitized oosparite, Sample $143-866 \mathrm{~A}-145 \mathrm{R}-1,82-84 \mathrm{~cm}$, scale $0.1 \mathrm{~mm}$. 7. Fracturing of pelecypod shell preceded sedimentation of micrite, framboidal pyrite (dark dots), Sample 143-866A-74R-2, 72-74 cm, scale 0.1 mm. 8. Phosphatized limestone with silicification (bright areas) and bladed and fibrous crystal forms of barite, Sample 143-867B-1R-1, 11-13 cm, scale 0.3 mm. 9. Large apatite crystals growing in distinct layers, matrix is penetratively phosphatized wackestone, Sample 143-867B-2R-2, 11-13 cm, scale $0.2 \mathrm{~mm}$. 